Somnologie 2020 24 (Suppl 1):S11-S48 (c) Springer Medizin Verlag GmbH, ein Teil von Springer Nature 2020

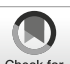
https://doi.org/10.1007/s11818-020-00267-4

\section{Abstracts der 28. Jahrestagung der Deutschen Gesellschaft für Schlafforschung und Schlafmedizin e.V.}

\author{
"Schlaf und Arbeit"
}

\author{
29.-31. Oktober 2020, virtuell
}

Veranstalter wissenschaftliches Programm

Deutsche Gesellschaft für Schlafforschung und Schlafmedizin (DGSM) e. V.

DGSM-Geschäftsstelle Hephata Klinik

Schimmelpfengstraße 6

34613 Schwalmstadt-Treysa

Wissenschaftliche Leitung

Prof. Dr. med. Helmut Frohnhofen

Universitätsklinikum Düsseldorf

Klinik für Orthopädie und Unfallchirurgie, Altersmedizin

Dr. med. Winfried Hohenhorst

Alfried Krupp Krankenhaus Rüttenscheid

Klinik für HNO-Heilkunde, Kopf- und Hals-Chirurgie

Prof. Dr. med. Georg Nilius

Kliniken Essen-Mitte

Pneumologie, Allergologie \& Zentrum für Schlafmedizin

Prof. Dr. med. Christoph Schöbel

Universitätsmedizin Essen

Schlafmedizinisches Zentrum der Ruhrlandklinik

Wissenschaftliches Komitee

Prof. Dr. Kneginja Richter (Nürnberg)

Prof. Dr. Christoph Schöbel (Essen)

Prof. Dr. Kai Spiegelhalder (Freiburg i. Br.)

Dr. med. Holger Hein (Reinbek)

Prof. Dr. Michael Arzt (Regensburg)

Prof. Dr. Angelika A. Schlarb (Bielefeld)

Dr. Sebastian Zaremba (Brunnen/CH)

Prof. Dr. med. Helmut Frohnhofen (Düsseldorf)

Dr. med. Winfried Hohenhorst (Essen)

Prof. Dr. med. Georg Nilius (Essen)

Wirtschaftlicher Veranstalter und Tagungsorganisation

Conventus Congressmanagement \& Marketing $\mathrm{GmbH}$

Carl-Pulfrich-Straße $1 \bullet 07745$ Jena

Telefon +49364131 16-305 • Fax +49364131 16-243

This supplement is not sponsored by industry. info@dgsm-kongress.de • www.dgsm-kongress.de 


\section{Freie Vorträge}

Auswirkungen eines Maskenwechsels auf die Nutzungsdauer der positiven Atemwegsdruck-Therapie: Analyse einer deutschen Gesundheitsdienstleister-Datenbank

\section{${ }^{*}$ M. Arzt ${ }^{1}$, C. Schoebel', J. H. Ficker ${ }^{3}$, A. Graml', J. Schnepf ${ }^{4}$, I. Fietze ${ }^{5}$, P. Young ${ }^{6}$, H. Wöhrle \\ ${ }^{1}$ Schlafmedizinisches Zentrum, Innere Medizin II, Regensburg, Deutschland; ${ }^{2}$ Universität Duisburg-Essen, Essen, Deutschland; ${ }^{3}$ Klinikum Nürnberg, Nürnberg, Deutschland; ${ }^{4}$ ResMed Science Center, Martinsried, Deutschland; ${ }^{5}$ Charité-Universitätsmedizin, Berlin, Deutschland; ${ }^{6}$ Medical Park, Abteilung für Neurologie, Bad Feilnbach, Deutschland; ${ }^{7}$ Schlaf- und Beatmungszentrum Blaubeuren, Lungenzentrum Ulm, Ulm, Deutschland}

Fragestellung: Bei Patienten mit mangelhafter Nutzung einer positiven Atemwegsdruck-Therapie (PAP) wird ein Maskenwechsel häufig in der klinischen Routine eingesetzt. Ziel der vorliegenden Analyse ist es daher, die Auswirkungen eines Maskenwechsels auf die PAP-Nutzung zu evaluieren.

Patienten und Methoden: Die Daten eines deutschen Gesundheitsdienstleisters (ResMed Healthcare Germany) wurden retrospectiv analysiert Patienten, die zwischen April 2014 und Juli 2018 mit der PAP-Therapie begonnen und erstmals ihren Maskentyp oder ihr Modell (Maskenverschiebung) gewechselt haben, wurden in die Analyse eingeschlossen ( $n=11.414$; erster Maskentyp: nasal- $38 \%$, Nasenpolster $13 \%$ und oronasal $49 \%$ ).

Ergebnisse: Bei Patienten mit niedriger PAP-Nutzung ( $\leq 4$ h/Tag, $22 \%$ ) war ein Wechsel der Maske mit einem stärkeren Anstieg der PAP-Adhärenz im Vergleich zu Patienten mit ausreichender PAP-Adhärenz $(>4 \mathrm{~h} /$ Tag, $78 \%: \Delta 30 \pm 108$ versus $\Delta-8 \pm 73 \mathrm{~min} / \mathrm{Tag}, p<0,001)$ assoziiert. $30 \%$ der Patienten mit niedriger PAP-Nutzung erreichten nach Wechsel der Maske eine ausreichende PAP-Nutzung. Der Wechsel von Nasenmaske zu einem anderen Maskentyp oder -modell (multiple binäre logistische Regressionsanalyse; OR [95\% CI]: 1,23 [1,02; 1,49]) sowie früher ( $<90$ Tage nach Einleitung der PAP-Therapie; $1,45[1,14 ; 1,87]$ ) oder später Wechsel des Maskentyps ( $>360$ Tage; $1,30[1,05 ; 1,60]$ ) waren signifikant mit dem Erreichen einer PAP-Nutzung $>4 \mathrm{~h} /$ Tag assoziiert. Alter, Versicherungsstatus, Art des PAP-Gerätyps, Leckage und Rest-AHI zeigten keinen relevanten Zusammenhang mit dem Erreichen einer PAP-Nutzung $>4 \mathrm{~h} / \mathrm{Tag}$ Schlussfolgerungen: Bei $30 \%$ der Patienten mit niedriger PAP-Nutzung kommt es nach Maskenwechsel noch zu einer ausreichenden PAP-Adhärenz von $>4 \mathrm{~h} /$ Tag. Der Wechsel von einer Nasenmaske zu einem anderen Masken-Typ oder Modell sowie ein Wechsel der Maske $<3$ Monate und $>1$ Jahr nach Einleitung der PAP-Therapie sind unabhängige Prädiktoren für das Erreichen einer ausreichenden PAP-Adhärenz.

Schlüsselwörter: CPAP, Nutzung, Interface, Compliance, Positivdruck

\section{3}

\section{Effects of short- and long-term solriamfetol treatment on} adherence to primary obstructive sleep apnoea therapy

P. Schweitzer ${ }^{1}$, K. P. Stroh ${ }^{2},{ }^{*}$ G. Mayer ${ }^{3,4}$, R. Rosenberg ${ }^{5,6}$, P. Chandler ${ }^{7}$, M. Baladi ${ }^{7}$, L. Lee ${ }^{7}$, A. Malhotra ${ }^{8}$

'Sleep Medicine and Research Center, St. Luke's Hospital, Chesterfield, United States; ${ }^{2}$ Case Western Reserve University, Cleveland, United States; ${ }^{3}$ Hephata Klinik, Schwalmstadt, Germany; ${ }^{4}$ Philipps University, Marburg, Germany; ${ }^{5}$ NeuroTrials Research, Inc., Atlanta, United States; ${ }^{6}$ Atlanta School of Sleep Medicine, Atlanta, United States; ${ }^{7}$ Jazz Pharmaceuticals, Palo Alto, United States; ${ }^{8}$ University of California San Diego Medical Center, Division of Pulmonary, Critical Care and Sleep Medicine, La Jolla, United States

Objectives: Solriamfetol is a dopamine/norepinephrine reuptake inhibitor approved in the EU and US to treat excessive daytime sleepiness (EDS) associated with obstructive sleep apnoea (OSA; 37.5-150 mg/day). This analysis evaluated whether treatment of EDS with solriamfetol affected primary OSA therapy device use.

Methods: Data from a 12-wk, randomized, double-blind, placebo-controlled phase 3 trial (NCT02348606) and an open-label extension (OLE) trial $\leq 52 \mathrm{wk}$ (NCT02348632) were analyzed. Participants received solriamfetol 37.5 (12-week study only), 75,150 , or $300 \mathrm{mg}$ /day or placebo (12week study only). Inclusion required current/prior primary OSA therapy use, including positive airway pressure (PAP), oral appliance, or surgical intervention. Primary OSA therapy use was recorded throughout the studies as electronically downloadable data (when available) or by diary. Baseline (BL) and end-of-study data are summarized for the safety populations. OLE data are reported for participants with OSA directly enrolled from a previous study; BL refers to BL in the parent study. Adverse events (AEs) are summarized.

Results: Primary OSA therapy use was reported for 344/474 participants in the 12-wk study (261 solriamfetol, 83 placebo) and 235/333 participants in the OLE; the majority were using PAP (12-wk study, 318 [92\%]; OLE, 222 [94\%]). In the 12-wk study, BL mean therapy across study groups was 89 $90 \%$ of nights, $6.6-6.7 \mathrm{~h} /$ night, and use $\geq 50 \% /$ night on $91-93 \%$ of nights; in the OLE, BL values were $90 \%$ of nights, $6.6 \mathrm{~h} /$ night, and use $\geq 50 \% /$ night on $90 \%$ of nights. In the 12 -wk study, mean \pm SD changes from BL to endpoint for participants on solriamfetol or placebo were $1.1 \pm 12.0 \%$ and $0.8 \% \pm 12.1 \%$ of nights, $-0.3 \pm 1.2$ and $-0.3 \pm 0.9 \mathrm{~h} /$ night, and use $\geq 50 \% /$ night on $2.2 \pm 13.7 \%$ and $2.7 \% \pm 19.4 \%$ of nights, respectively. OLE mean changes ranged from $-0.5 \%$ to $2.0 \%$ of nights, -1.0 to $-0.7 \mathrm{~h} / \mathrm{night}$, and use $\geq 50 \%$ /night on $3.6-6.5 \%$ of nights. Common AEs ( $\geq 5 \%$ in either study) with solriamfetol were headache, nausea, decreased appetite, anxiety, nasopharyngitis, diarrhoea, dry mouth, insomnia, and upper respiratory infection.

Conclusions: Treatment of EDS with solriamfetol did not affect primary OSA therapy use throughout the 12 -wk or OLE study. Safety was similar between studies.

Keywords: JZP-110, OSA, Compliance, Airway obstructive, Sleep-disordered breathing

\section{Sleep health and brain morphometry in the UK biobank}

*J. E. Schiel', S. Tamm ${ }^{2,3}$, C. E. Sexton ${ }^{3,4}$, B. Feige', D. Riemann', M. K. Rutter ${ }^{5,6}$, S.D.Kyle , K. Spiegelhalder

'Universitätsklinikum Freiburg, Klinik für Psychiatrie und Psychotherapie, Freiburg i. Br., Germany; ${ }^{2}$ Karolinska Institutet, Department of Clinical Neuroscience, Stockholm, Sweden; ${ }^{3}$ University of Oxford, Department of Psychiatry, Oxford, United Kingdom; ${ }^{4}$ Global Brain Health Institute, Department of Neurology, San Francisco, United States; ${ }^{5}$ University of Manchester, Faculty of Biology, Manchester, United Kingdom; ${ }^{6}$ Manchester Academic Health Science Centre, Manchester Diabetes Centre, Manchester, United Kingdom; ${ }^{7}$ University of Oxford, Nuffield Department of Clinical Neurosciences, Oxford, United Kingdom

Objectives: Sleep is a complex physiological process regulated by various brain circuits and neurotransmitter systems. Empirical research has clearly demonstrated that different aspects of sleep health are related to future morbidity and mortality. However, it is unclear whether neurostructural abnormalities underlie different aspects of sleep health. Several small-scale cross-sectional case-control studies have supported this hypothesis; however, the results so far are somewhat inconclusive. Thus, in light of recent doubts about replicability and low power in neuroimaging studies, the current study seeks to investigate the independent associations between several sleep health variables and brain morphometry in a large sample of individuals of the UK Biobank (UKBB, current results are based on a sample of $n=16,704$; results presented in Sevilla will be based on an updated sample of $n=39,691$ ).

Methods: Cross-sectional data from the UK Biobank (Sudlow et al., 2015) was analyzed using multiple linear regression models with sleep health variables (insomnia symptoms, sleep duration, daytime sleepiness and chronotype) as concurrently included independent variables. Covariates 
Hier steht eine Anzeige.

\section{算 Springer}


comprised sleep medication use, psychopharmacological treatment, socioeconomic status, depressive symptoms and sleep apnoea. Dependent variables were regional grey matter volumes of 139 brain areas.

Results: All analyses were based on a Bonferroni-adjusted significance level. Short sleep duration was found to be associated with reduced grey matter volume in the right precuneus $(-0.7 \%, p=0.0003)$ and increased grey matter volume in the 10th lobule of the cerebellar vermis $(+1.3 \%$, $p=0.0001)$. No other sleep health variables were related to brain morphometry.

Conclusions: The current results suggest a negative association between subjective short sleep duration and grey matter volume in the right precuneus-and a positive association between subjective short sleep duration and grey matter volume of the 10th lobule of the cerebellar vermis. The former finding might be related to altered DMN connectivity in patients with short sleep duration. However, further sleep health variables like insomnia symptoms and daytime sleepiness were not associated with changes in brain morphometry.

Keywords: Sleep health, Brain morphometry, UK Biobank, Sleep duration, Grey Matter volume

\section{Zusammenhang zwischen REM-Schlaf und der Körperfettverteilung bei Patienten mit Insomnie}

*S. L. Weinhold', C. Blome', M. Wittich', J. Lechinger', W. Braun ${ }^{1,2}$, M. Müller', A. Bosy-Westphal', R. Göder ${ }^{r}$

'UKSH Campus Kiel, Zentrum für Schlafmedizin, Kiel, Deutschland; ${ }^{2}$ Institut für Humanernährung der CAU, Kiel, Deutschland

Fragestellung: Epidemiologische Studien weisen darauf hin, dass Schlafdauer und -qualität mit der Entstehung von Übergewicht zusammenhängen könnten (z. B. Taheri et al., 2004). Die Verteilung des Köperfetts wurde bis jetzt nur von wenigen Studien berücksichtigt. Ziel dieser Studie ist es, den Zusammenhang von Schlafparametern und der Körperzusammensetzung bei Patienten mit Insomnie zu untersuchen.

Patienten und Methoden: Eingeschlossen wurden neun Insomniepatientinnen (Alter: $44 \pm 12$ Jahre, BMI: $23,2 \pm 3,8 \mathrm{~kg} / \mathrm{m}^{2}$ ). Die Untersuchungen im Rahmen der Studie umfassten zwei Nächte Polysomnographie, die Messung der Fettmasse und der fettfreien Masse mittels Air Displacement Plethysmography, sowie eine Messung des subkutanen und viszeralen Fettanteils mittels abdominaler Magnetresonanztomographie (MRT). Ergebnisse: Die durchschnittliche Schlafdauer betrug $379 \pm 38 \mathrm{~min}$, die REM-Schlafdauer $64 \pm 21$ min und die Schlafeffizienz $87 \pm 7 \%$. Die Fettmasse lag bei $21,4 \pm 6,9 \mathrm{~kg}$ und die fettfreie Masse bei $45,2 \pm 4,9 \mathrm{~kg}$. Die MRT-Untersuchung ergab einen viszeralen Fettanteil von $0,5 \pm 0,3$ Litern und einen subkutanen Fettanteil von 13,9 44,4 Litern. Das Hauptergebnis war eine signifikante negative Partialkorrelation (Kontrolliert für Alter) der subkutanen $(r=-0,7)$ und viszeralen Fettmasse $(r=-0,9)$ mit der REMSchlafdauer, nicht aber mit nonREM-Schlaf Parametern.

Schlussfolgerungen: Diese Ergebnisse weisen darauf hin, dass vor allem die REM-Schlafdauer bei der Entwicklung von Übergewicht bei Insomnie eine Rolle spielen könnte. Obwohl diese Studie keine Aussage zur Kausalität erlaubt, passt dieser Befund z. B. dazu, dass partieller Schlafentzug bei gesunden Probanden zu einer Reduktion des Hormons Leptin führt, welches eine wichtige Rolle bei der Hungerregulierung und dem Fettstoffwechsel spielt (Spiegel et al., 2004). So könnten diejenigen Insomniepatienten mit einem geringeren REM-Schlafanteil durch beispielsweise einen niedrigen Leptinspiegel mehr Nahrung aufnehmen bzw. mehr Fett speichern.

Schlüsselwörter: Insomnie, Übergewicht, Körperfettverteilung, REMSchlaf, Schlafstadien

\section{Literatur}

1. Taheri S, Lin L, Austin D, Young T, Mignot E (2004) Short sleep duration is associated with reduced leptin, elevated ghrelin, and increased body mass index. PLoS Med 1:e62
2. Spiegel K, Leproult R, L'hermite-Baleriaux M, Copinschi G, Penev PD, van Cauter $E$ (2004) Leptin levels are dependent on sleep duration: relationships with sympathovagal balance, carbohydrate regulation, cortisol, and thyrotropin. J Clin Endocrinol Metab 89:

Therapiesteuerung basierend auf der Baveno-Klassifikation, angewandt in der ESADA-Kohorte

${ }^{*}$ W. J. Randerath ${ }^{1}$, ${ }^{*}$. Herkenrath', M. Treml', L. Grote' ${ }^{2}$, J. Hedner', M. Bonsignore ${ }^{3}$, J. L. Pépin ${ }^{4}$, S. Ryan ${ }^{5}$, S. Schiza 6 , J. Verbraecken7, W. McNicholas ${ }^{5}$, A. Pataka ${ }^{8}$, P. Sliwinski ${ }^{9}$, Ö. Basoglu ${ }^{10}$

'Institut für Pneumologie an der Universität zu Köln, Solingen, Deutschland; ${ }^{2}$ Sahlgrenska University Hospital, Department of Sleep Medicine, Respiratory Medicine and Allergology, Göteborg, Schweden; ${ }^{3 P R O M I S E}$ Department, University of Palermo and IRIB-CNR, Palermo, Italien; ${ }^{4} \mathrm{HP} 2$ Laboratory, INSERM U1042, Grenoble Alpes University and EFCR Laboratory, Thorax and Vessels Division, Grenoble Alpes University Hospital, Grenoble, Frankreich; ${ }^{5}$ St Vincent's University Hospital and School of Medicine, University College Dublin, Department of Respiratory and Sleep Medicine, Dublin, Irland; ${ }^{6}$ University of Crete, Department of Thoracic Medicine, Sleep Disorders Center, Heraklion, Griechenland; ${ }^{7}$ Antwerp University Hospital and University of Antwerp, Multidisciplinary Sleep Disorders Centre, EdegemAntwerp, Belgien; ${ }^{8} \mathrm{G}$. Papanikolaou Hospital, Respiratory Failure Unit, Thessaloniki, Griechenland; ${ }^{9}$ nstitute of Tuberculosis and Lung Diseases, 4th Department of Respiratory Medicine, Warschau, Polen; ${ }^{10}$ Ege University School of Medicine, Department of Chest Diseases, Izmir, Türkei

Fragestellung: Neue Erkenntnisse stellen die Bedeutung des Apnoe-Hypopnoe-Index (AHI) für die Klassifizierung der obstruktiven Schlafapnoe (OSA) in Frage. Kürzlich wurde ein System zur OSA-Klassifizierung und der Therapiesteuerung vorgeschlagen, welches Symptomatik und Komorbiditäten integriert (Baveno, - Abb. 1a). Wir untersuchten, ob sich durch diese Klassifikation gegenüber der klassischen AHI-basierten Therapiesteuerung Vorteile ergeben.

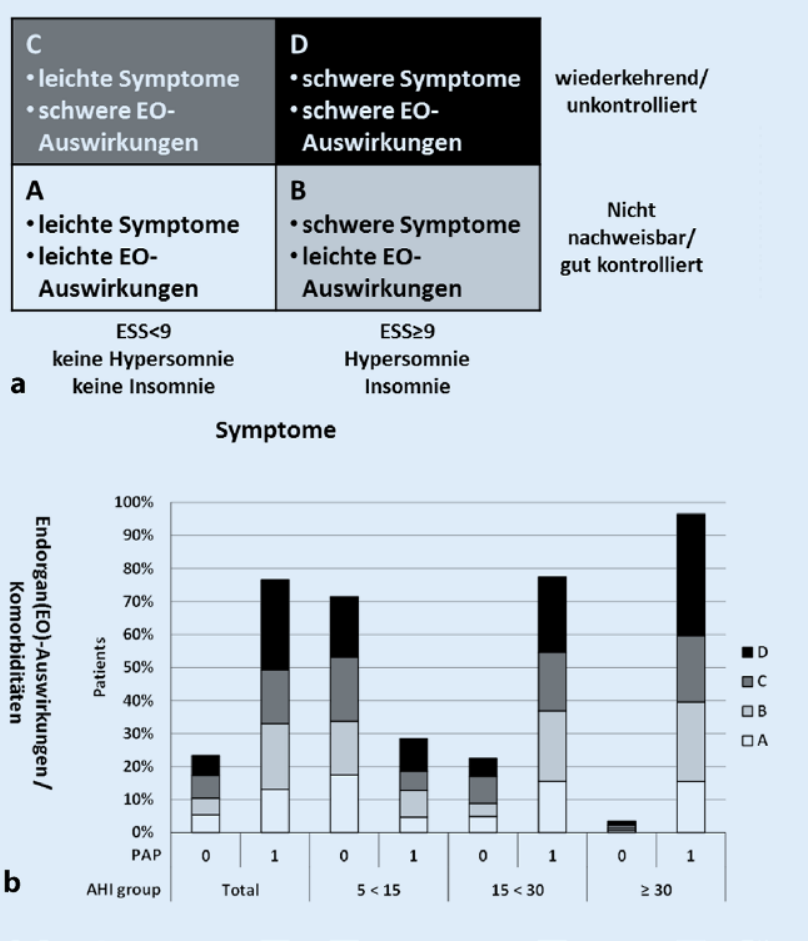

Abb. $1 \mid 6$ 


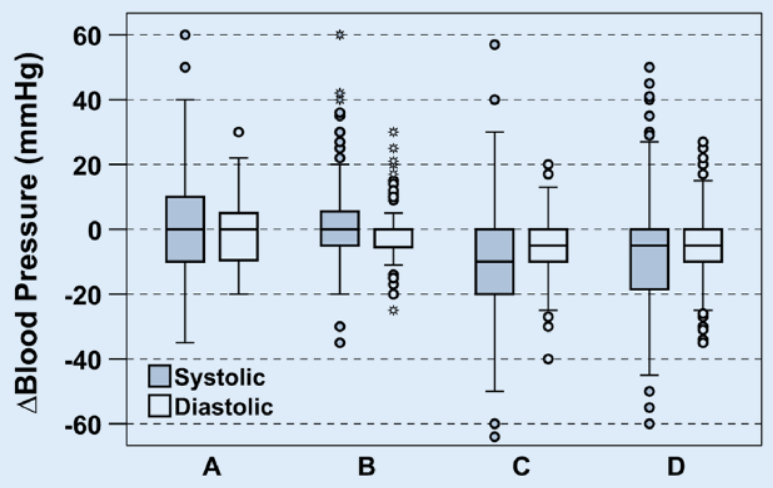

a

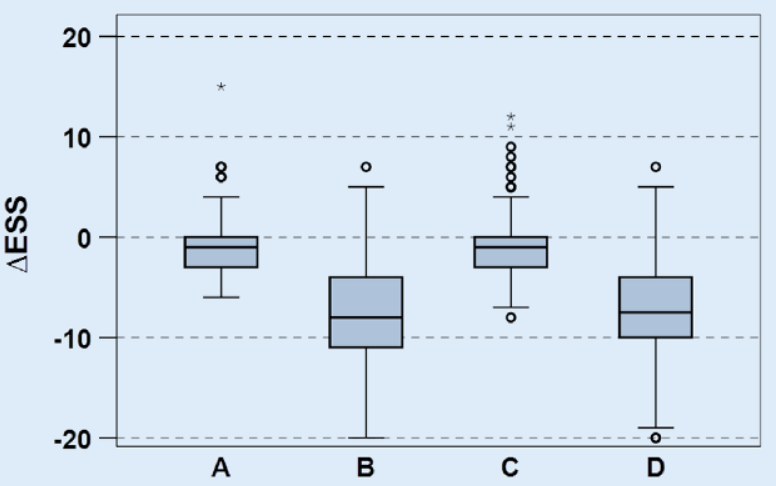

b

Baveno Group

\section{7}

\section{Schlafmedizinische Unterschiede bei Anwendung der Baveno- Klassifikation in der ESADA-Kohorte}

*W. J. Randerath', *S. Herkenrath', M. Treml', L. Grote', J. Hedner', M. Bonsignore', J. L. Pépin', S. Ryan ${ }^{5}$, S. Schiza ${ }^{6}$, J. Verbraecken',

W. McNicholas ${ }^{5}$, A. Pataka ${ }^{8}$, P. Sliwinski ${ }^{9}$, Ö. Basoglu' ${ }^{10}$

'Institut für Pneumologie an der Universität zu Köln, Solingen, Deutschland; ${ }^{2}$ Sahlgrenska University Hospital, Department of Sleep Medicine, Respiratory Medicine and Allergology, Göteborg, Schweden; ${ }^{3}$ PROMISE Department, University of Palermo and IRIB-CNR, Palermo, Italien; ${ }^{4} \mathrm{HP} 2$ Laboratory, INSERM U1042, Grenoble Alpes University and EFCR Laboratory, Thorax and Vessels Division, Grenoble Alpes University Hospital, Grenoble, Frankreich; ${ }^{5}$ St Vincent's University Hospital and School of Medicine, University College Dublin, Department of Respiratory and Sleep Medicine, Dublin, Irland; ' 6 niversity of Crete, Department of Thoracic Medicine, Sleep Disorders Center, Heraklion, Griechenland; ${ }^{7}$ Antwerp University Hospital and University of Antwerp, Multidisciplinary Sleep Disorders Centre, EdegemAntwerp, Belgien; ${ }^{8} \mathrm{G}$. Papanikolaou Hospital, Respiratory Failure Unit, Thessaloniki, Griechenland; ${ }^{9}$ Institute of Tuberculosis and Lung Diseases, 4th Department of Respiratory Medicine, Warschau, Polen; ${ }^{10}$ Ege University School of Medicine, Department of Chest Diseases, Izmir, Türkei

Fragestellung: Neue Erkenntnisse stellen die Bedeutung des Apnoe-Hypopnoe-Index (AHI) für die Klassifizierung der obstruktiven Schlafapnoe (OSA) in Frage. Kürzlich wurde ein neuartiges Klassifikationssystem für den Schweregrad der OSA vorgeschlagen („Baveno-Klassifikation“, - Abb. 1). Es umfasst die Symptombelastung und die Auswirkungen auf die Endorgane, um die Patienten in vier Gruppen zu klassifizieren. In der aktuellen Studie wurde diese Klassifikation in einer großen repräsentativen Patientenkohorte angewandt, um die Fähigkeit der Abgrenzung verschiedener OSA-Phänotypen zu evaluieren und schlafmedizinische Gruppenunterschiede zu prüfen.

Patienten und Methoden: OSA-Patienten aus der Kohorte der European Sleep Apnoea Data Base (ESADA), die ab 2007 in 20 Ländern erhoben wurde, wurden nach Symptomen (Epworth Sleepiness Scale Score $\geq 9$, subjektive Schlafdauer $\geq 11 \mathrm{~h}$, Diagnose Schlaflosigkeit) und den wichtigsten Auswirkungen auf die Endorgane (unkontrollierter arterieller Bluthochdruck, Vorhofflimmern, Herzinsuffizienz, Diabetes und Schlaganfall) kategorisiert.

Ergebnisse: 14.499 Patienten ( $28 \%$ weibl, $54 \pm 12$ Jahre, BMI $33 \pm 7 \mathrm{~kg} / \mathrm{m}^{2}$ ) wurden kategorisiert. Die Baveno-Klasse A-D erlaubt eine Abgrenzung verschiedener OSA-Phänotypen, die sich auch hinsichtlich schlafmedizinische Risikomarker unterscheiden. Die T90 war bei entweder schweren Symptomen oder schwerwiegenden Endorgan-Auswirkungen genauso wie der AHI erhöht. Beide Parameter steigen noch weiter an, wenn Tagesschläfrigkeit und Endorganschäden koexistierten (• Tab. 1). So lässt sich jede einzelne Gruppe durch die verschiedenen klinischen Parameter einerseits und die hypoxische Last andererseits abgrenzen.

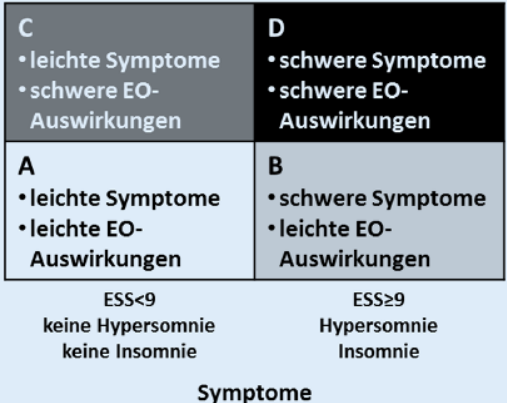

wiederkehrend/ unkontrolliert

Schlussfolgerungen: Die Klassifikation nach Baveno erlaubt tatsächlich die unmittelbare Identifikation jener Patienten, die prognostisch und/oder symptomatisch von einer Therapie profitieren. Da alle Bavenogruppen in den verschiedenen AHI-Schweregraden mit relevantem Anteil vertreten sind zeigt sie umgekehrt aber auch, dass eine Therapiesteuerung alleinig orientiert am AHI jene Patienten mit milder Schlafapnoe der Gruppen B, C, D unbehandelt ließe, die das Potenzial einer symptomatischen und/ oder prognostischen Besserung aufweisen. Die Bavenoklassifikation erlaubt weit besser als der AHI verschiedene OSA-Phänotypen abzubilden und das therapeutische Procedere festzulegen.

Schlüsselwörter: Obstruktive Schlafapnoe, Baveno-Klassifikation, ESADA, Tagesschläfrigkeit, Blutdruck 


\begin{tabular}{|c|c|c|c|c|c|}
\hline Baveno-Gruppe & A & B & C & D & \\
\hline$n / \%$ & $2656 / 18 \%$ & $3562 / 25 \%$ & $3396 / 23 \%$ & $4885 / 34 \%$ & - \\
\hline Alter & $54 \pm 13$ & $52 \pm 12$ & $56 \pm 13$ & $55 \pm 12$ & Alle $<0,05$ \\
\hline REM (\%) & $14 \pm 8$ & $14 \pm 8$ & $13 \pm 8$ & $12 \pm 8$ & Alle $<0,05$ \\
\hline T90 (min) & $35 \pm 61$ & $46 \pm 74$ & $44 \pm 75$ & $67 \pm 88$ & $\begin{array}{l}\text { Alle }<0,001 \text { außer B vs. } \\
C(0,30)\end{array}$ \\
\hline $\mathrm{AHI}(/ \mathrm{h})$ & $32 \pm 22$ & $37 \pm 25$ & $34 \pm 23$ & $42 \pm 28$ & Alle $<0,05$ \\
\hline
\end{tabular}

Schlussfolgerungen: Unsere Daten zeigen, dass die „Baveno“-Klassifikation tatsächlich in der Lage ist, vier verschiedene Phänotypen mit relevanter Häufigkeit in einer repräsentativen europäischen Patientenkohorte abzugrenzen. Unterschiede in der T90, die bekanntermaßen mit schlechten kognitiven, kardiovaskulären und metabolischen Ergebnissen korreliert, lässt ein unterschiedliches Outcome der Bavenogruppen vermuten. Dies sollte in einer prospektiven Multicenter-Studie überprüft werden.

Schlüsselwörter: Obstruktive Schlafapnoe, Baveno-Klassifikation, ESADA, Hypoxische Last, AHI

\section{8}

\section{Schlaf, Wohlbefinden, Arbeit und beruflicher Stress während der COVID-19 Pandemie}

\section{${ }^{*}$ C. Saalwirth}

Universität der Bundeswehr München, Institut für Psychologie:

Entwicklungs- und Gesundheitspsychologie, Neubiberg, Deutschland

Fragestellung: Berufstätige wurden durch COVID-19 vor neue Herausforderungen, wie beispielsweise vermehrtes Home-Office, gestellt. Mit dieser Studie wird daher untersucht, welchen Einfluss Arbeitsstress, HomeOffice, ein variabler Arbeitsbeginn und das Wohlbefinden auf den Schlaf in Corona-Zeiten haben und ob sich dieser im Vergleich zur Zeit vor der COVID-19 Pandemie verändert hat.

Patienten und Methoden: Alle Daten wurden mit einem Online-Fragebogen erhoben, den 320 berufstätige Teilnehmer, die keiner Schichtarbeit nachgingen, bearbeiteten (51,9\% Männer, 48,1 \% Frauen; 19-68 Jahre). Neben soziodemografischen Daten und Angaben zu Home-Office und variablem Arbeitsbeginn $(\mathrm{AB})$ wurden Arbeitsstress mit zwei Subskalen des Trierer Inventars zu chronischem Stress (TICS), Negativer und Positiver Affekt (NA, PA) mit der PANAS sowie die Schlafqualität (SQ) und Schlafdauer (SD) mit adaptierten Items des PSQIs erfasst. Zusätzlich wurde mit jeweils einem Item erfragt, ob sich die SQ bzw. SD im Vergleich zu vor COVID-19 verändert hat. Ergebnisse: $20 \%$ bzw. 13,7\% der Teilnehmer gaben an, seit COVID-19 schlechter bzw. kürzer zu schlafen, $11 \%$ bzw. $24,7 \%$ gaben an besser bzw. länger zu schlafen. Berufstätige im Home-Office weisen einen variableren $\mathrm{AB}$ auf $\left(\mathrm{r}=0,16^{* *}\right)$ und schlafen im Vergleich zur Zeit vor COVID-19 länger $\left(-0,13^{* *}\right)$. Ein variabler $\mathrm{AB}$ ist mit einer schlechteren SQ $\left(r=-0,12^{*}\right)$ verbunden, hat aber keinen Einfluss auf die SD. Die SQ weist zudem Zusammenhänge mit Arbeitsstress $\left(r=-0,30^{* *}\right)$, NA $\left(r=-0,36^{* *}\right)$ und PA $\left(r=0,34^{* *}\right)$ auf, die SD lediglich einen Zusammenhang mit Arbeitsstress $\left(\mathrm{r}=-0,18^{\star *}\right)$. Es zeigt sich weiter, dass eine höhere SQ mit einer längeren SD einhergeht $\left(r=0,27^{* *}\right)$. Bei der linearen Regression zur Vorhersage der SD durch Arbeitsstress und SQ erwies sich nur die SQ als signifikanter Prädiktor (- Abb. 1). Die Ergebnisse der linearen Regression zur Vorhersage der SQ durch PA, NA, Arbeitsstress, variabler AB und SD zeigen, dass alle Prädiktoren, außer der Variable AB, einen signifikanten Einfluss auf die SQ aufweisen (• Abb. 2).

Tab. 1

\begin{tabular}{lcc}
\hline Prädiktor & $\boldsymbol{\beta}$ & $\mathrm{p}-$ Wert \\
\hline Arbeitsstress & -.09 & .10 \\
Schlafqualität & .26 & $.00^{\star \star}$ \\
\hline Anmerkungen: Kriterium $=$ Schlafdauer, $R^{2}=.09,{ }^{*} \mathrm{p}<0.05, \mathrm{p}^{\star \star}<0.01$
\end{tabular}

Tab. 2

\begin{tabular}{lcc}
\hline Prädiktor & $\boldsymbol{\beta}$ & p-Wert \\
\hline Positiver Affekt & .30 & $.00^{\star *}$ \\
Negativer Affekt & -.22 & $.00^{\star *}$ \\
Arbeitsstress & -.20 & $.00^{\star *}$ \\
Variabler Arbeitsbeginn & -.03 & .49 \\
Schlafdauer & .26 & $.00^{\star *}$ \\
\hline Anmerkungen: Kriterium $=$ Schlafqualität, $R^{2}=.31,{ }^{*} \mathrm{p}<0.05, \mathrm{p}^{* \star}<0.01$
\end{tabular}

Abb. $1 \mid 8$ 
Schlussfolgerungen: Die Veränderungen durch COVID-19 weisen im Hinblick auf den Schlaf sowohl positive als auch negative Entwicklungen auf. Beeinflusst wird die subjektive Schlafqualität durch den PA, den NA, den Arbeitsstress und die SD. Die SD wiederum wird nur durch die SQ maßgeblich vorhergesagt.

Schlüsselwörter: Schlafqualität, Schlafdauer, Wohlbefinden, Arbeitsstress, COVID-19

\section{9}

Langzeitwirkungen von Fluglärm auf die subjektive Schlafqualität und die Belästigung von Grundschulkindern: Vergleich der Kinder- und Elternbefragungen aus der Studie MIDAS

*J. Quehl, S. Bartels, D. Aeschbach

Deutsches Zentrum für Luft- und Raumfahrt, Institut für Luft- und Raumfahrtmedizin, Schlaf und Humanfaktoren, Köln, Deutschland

Fragestellung: Da Grundschulkinder zu Zeiten schlafen, zu denen nächtliche Verkehrsströme und Lärmbelastungen noch relativ hoch sind und sie zudem weniger adäquate Lärmbewältigungsstrategien als Erwachsene besitzen, wird ihr Schlaf als gefährdet gegenüber nächtlichen Verkehrslärmwirkungen eingestuft. Bisherige Studien zu chronischen Verkehrslärmwirkungen auf die Schlafqualität und Belästigung bei Kindern basierten im Wesentlichen auf Elternbefragungen und ließen Selbsteinschätzungen von Kindern außer Acht. Patienten und Methoden: In einer Feldstudie am Flughafen Köln/Bonn mit $N=51$ Grundschulkindern (8-10 Jahre, 23 Mädchen) wurden Kinder- und Elternbefragungen zu den Auswirkungen chronischen Fluglärms im häuslichen Wohnumfeld auf die subjektive Schlafqualität von Kindern sowie die resultierende Langzeitbelästigung und allgemeine Wohnzufriedenheit durchgeführt. Die Lärmempfindlichkeit und Einstellungen zum Flugverkehr als wichtige moderierende Einflussgrößen der Belästigung wurden ebenfalls erhoben. Anhand der Befragungsdaten wurde untersucht, inwieweit sich Kinder und Eltern in ihren Einstellungen zum Flugverkehr, der Fluglärmbelästigung und allgemeinen Wohnzufriedenheit unterscheiden bzw. inwieweit die Bewertungen zusammenhängen.

Ergebnisse: Für den kindlichen Schlaf gaben sowohl Kinder als auch Eltern Störungen des Einschlafens als häufigste Beeinträchtigung an. In der Selbsteinschätzung der Fluglärmbelästigung, Lärmempfindlichkeit, Wohnzufriedenheit und des Flugverkehrs unterschieden sich Kinder und Eltern in multiplen t-Tests statistisch signifikant $(p \leq 0,050)$. Die Kinder waren signifikant weniger durch den Fluglärm der vorausgegangenen 12 Monate belästigt und stuften sich signifikant weniger lärmempfindlich ein. Sie bewerteten den Flugverkehr signifikant positiver und waren signifikant zufriedener mit ihrer Wohngegend. Während die Belästigungsurteile mit $\mathrm{r}=0,980(p=0,004)$ zwischen Kinder und Eltern signifikant interkorreliert waren, hingen die Bewertungen der Lärmempfindlichkeit $(\mathrm{r}=-0,235, p=0,097)$ und Wohnzufriedenheit $(\mathrm{r}=-0,065, p=0,651)$ nicht zusammen. Gleiches galt für die Einstellungen zum Flugverkehr.

Schlussfolgerungen: Kinder und Eltern unterscheiden sich in der Wahrnehmung und Bewertung möglicher Auswirkungen von Fluglärm signifikant. Anders als bei der Langzeitbelästigung hängen die Bewertungen des Flugverkehrs und der Wohngegend nicht zusammen. Einstellungen und Erwartungen von Eltern scheinen sich demnach nicht zwangsläufig auf ihre Kinder zu übertragen.

Schlüsselwörter: Nachtfluglärm, Kinder, Langzeitwirkungen, Schlafqualität, Belästigung

\section{Hier steht eine Anzeige.}


10

\section{Schlaftraining für Säuglinge und Kleinkinder (Mini-KiSS) - was zeigen erste Langzeitergebnisse nach einem Jahr in Bezug auf frühkindliche Regulationsproblematiken?}

\author{
*M. Schnatschmidt, F. Lollies, A. A. Schlarb \\ Universität Bielefeld, Klinische Psychologie und Psychotherapie des Kindes- \\ und Jugendalters, Bielefeld, Deutschland
}

Fragestellung: Frühkindliche Regulationsstörungen umfassen im Wesentlichen problematische Verläufe beim Schlafen, Schreien und Essverhalten der Kinder. Schlafen ist lebenswichtig und unentbehrlich für das körperliche und psychische Wohlbefinden sowie eine gesunde Entwicklung. Einschlafstörungen und Durchschlafstörungen sind typische Probleme im Säuglings- und Kleinkindalter, häufig verbunden mit Schreiattacken. Schlafstörungen in diesem Alter neigen zur Chronifizierung und gehen häufig mit elterlicher Belastung einher, weshalb eine Intervention in diesem frühen Alter sehr wichtig ist. Diese Studie evaluiert ein multimodales Trainingsprogramm für Eltern von Kindern zwischen 0,5 und 4 Jahren mit Schlafstörungen (Mini-KiSS) und untersucht im Besonderen die Fragestellung, ob sich das Trainingsprogramm über die Schlafproblematik hinaus auf die frühkindliche Regulationsfähigkeit auswirkt und wie diese Problembereiche sich ein Jahr nach dem Training im follow-up darstellen. Patienten und Methoden: Es werden Daten von Eltern und deren Kindern erhoben. Zur Erfassung des kindlichen Schlafverhaltens führen die Eltern vor und nach dem Trainingsprogramm ein Schlaftagebuch und füllen zu jedem Messzeitpunkt spezifische Fragebögen (CSHQ, SFS) aus, durch die sowohl Schlafverhalten als auch frühkindliche Regulationsstörungen und Aspekte der kindlichen Emotionsregulation erfasst werden.

Ergebnisse: Die Wirksamkeit des Trainings (Mini-KiSS) wird basierend auf den verschiedenen spezifischen Instrumenten detailliert vorgestellt. Beleuchtet werden vor allem Effekte der Intervention auf den kindlichen Schlaf, sowie auf die frühkindliche Regulationsfähigkeit, mit besonderem Fokus auf frühkindliche Regulationsstörungen (wie exzessives Schreien und Fütterstörungen). Es soll ein besonderer Fokus auf die Ergebnisse ein Jahr nach dem Training gelegt werden, um erste Langzeiteffekte darstellen zu können.

Schlussfolgerungen: Wie in bisherigen Studien gezeigt werden konnte, hat das Mini-KiSS Schlaftraining einen positiven Einfluss auf den kindlichen Schlaf. Außerdem konnte eine Pilotstudie zeigen, dass sich über das kindliche Schlafverhalten hinaus auch die Regulationsfähigkeit der Kinder, vor allem das Schreiverhalten wesentlich verbesserte. Ein langfristiger Effekt über ein Jahr nach dem Training hat vor allem im Hinblick auf die Versorgungslage der Säuglinge und Kleinkinder Relevanz, da durch eine Intervention dieser Art schon früh Problematiken begrenzt werden könnten und psychisches Wachstum gewährleistet werden kann.

Schlüsselwörter: Insomnie, Säuglinge und Kleinkinder, Regulationsprobleme, Intervention, 1 Jahres Follow up

\section{1}

\section{Neurophysiologie der NREM Parasomnien}

${ }^{*}$ G. Mayer' ${ }^{1}$ A. Heidbreder ${ }^{2}$, D. Krefting ${ }^{3}$

'Philipps Universität Marburg, Neurologie, Marburg, Deutschland; ${ }^{2}$ Universitätsklinik für Neurologie Innsbruck, Neurologie, Innsbruck, Österreich; ${ }^{3}$ nstitut für Medizinische Informatik, Göttingen, Deutschland

Fragestellung: Die Pathophysiologie der NREM Parasomnien ist nicht ausreichend erforscht. Die Studienziele beinhalteten die Untersuchung der Polysomnographien einer Kohorte von Patienten mit NREM Parasomnien, um Hypothesen zur Pathophysiologie aus den letzten Jahren überprüfen zu können: Fragmentierung der ersten Schlafzyklen, verzögerter Auf- und Abbau des langsamwelligen Schlafs, Zunahme der langsamwelligen Aktivität vor den parasomnischen Ereignissen, Topographie und neuronale Netzwerke, Unterschiede der Ereignisse abhängig von den in der Folge auftretenden Stadienwechseln (Wach oder Schlaf).

Patienten und Methoden: Polysomnographien von 196 Schlafwandlern (gem. ICSD 2/3 Kriterien) wurden mit Polysomnographien von 110 al- ters- und geschlechtsgleichen Kontrollen der SIESTA Gruppe verglichen. Die Schlafstadien Bestimmung erfolgte gem. Rechtschaffen \& Kales. Time delay stability (TDS) wurde zur Bestimmung der Gehirn Konnektivität verwendet.

Ergebnisse: Schlafwandler hatten mehr Tiefschlaf N3, keine Änderung der N3 Latenz, N3 nahm altersabhängig zu, die N3 Phasen waren vermindert aber länger als bei Kontrollen. Die Wachzeit nach Schlafbeginn war in den ersten Schlafzyklen verlängert, die Anzahl der Wachphasen in der Nacht nichtsignifikant vermehrt, der Übergang von N3 zu Wach war vermehrt und von N3 zu N2 verringert. Es fanden sich mehr Transitionen von N2N3-Wach und Wach-N2. Die TDS Konnektivität zeigte bei Schlafwandlern mehr verstärkte „link ratios“ zwischen frontalen und zentralen und zentralen und okzipitalen Regionen. Die TDS Konnektivität 3 und 6 min vor Ereignissen zeigten eine Tendenz zu geringeren „link ratios“ in der Domäne fronto-okzipital niedriger Frequenzen.

Schlussfolgerungen: Unsere Daten bestätigen bei Schlafwandlern eine höhere N3 Stabilität, einen höheren N3 Schlafdruck, eine erhöhte Anzahl von Arousal aus N3, ein dissoziierte fronto-okzipitale Konnektivität und vermehrte Wachphasen im Schlaf als bei Kontrollen.

Schlüsselwörter: Parasomnie, NREM, Physiologie, Zerebrale Konnektivität, Tiefschlaf

\section{2}

\section{Der Einfluss nächtlicher Straßenverkehrslärmbelastung und Arbeitsbelastung auf die Prävalenz von subjektiv schlechtem Schlaf}

*S. Bartels', M. Ögren², J. L. Kim², S. Fredriksson², K. Persson Waye²

'Deutsches Zentrum für Luft- und Raumfahrt, Institut für Luft- und Raumfahrtmedizin, Schlaf und Humanfaktoren, Köln, Deutschland; ${ }^{2}$ Universität Göteborg, Sahlgrenska Academy, Occupational and Environmental Medicine, Göteborg, Schweden

Fragestellung: Nächtlicher Straßenverkehrslärm gilt als eine Quelle für Schlafstörungen. In epidemiologischen Studien zeigte sich wiederholt ein Effekt nächtlicher Verkehrslärmbelastung auf die selbsteingeschätzte Schlafqualität, wobei die Größe des Effekts stark schwankte. Die Arbeitsbelastung ist ebenfalls eine wichtige Determinante der Schlafqualität, ging in bisherigen Verkehrslärmstudien jedoch kaum als zu kontrollierende Variable ein. Die vorliegende Arbeit untersucht den Einfluss von Straßenverkehrslärm und Arbeitsbelastung sowie potenziellen Synergieeffekten auf subjektiv schlechten Schlaf.

Patienten und Methoden: Schlechter Schlaf und dessen potenzielle Determinanten wurden in einer Befragung mit 3955 erwerbstätigen Frauen aus der schwedischen Provinz Västra Götaland erhoben. Arbeitsbelastung wurde durch die Konstrukte Job Strain und Effort-Reward-Imbalance (ERI) operationalisiert. Die nächtliche Straßenverkehrslärmbelastung wurde zum einen durch die Ausrichtung des Schlafzimmerfensters hin zu einer a) ruhigen Fassade ohne Straße oder zu einer Straße mit b) geringem, c) mittlerem oder d) hohem Verkehrsaufkommen und zum anderen durch den modellierten energieäquivalenten Dauerschallpegel vor dem Haus für die Nachtzeit $\left(L_{22-6}\right)$ erfasst. Hierbei wurde unterschieden zwischen niedriger $(<45 \mathrm{~dB}(\mathrm{~A}))$, mittlerer $(45-50 \mathrm{~dB}(\mathrm{~A}))$ und hoher Lärmbelastung $(>50 \mathrm{~dB}(\mathrm{~A}))$. Der Einfluss der Lärm- und Arbeitsbelastung wurde durch multiple logistische Regressionsanalysen unter Kontrolle persönlicher und sozioökonomischer Variablen ermittelt. Synergieeffekte wurden mittels Testung auf additive Interaktion bestimmt.

Ergebnisse: Mit steigender Arbeitsbelastung (Job Strain und ERI) stieg die Prävalenz von subjektiv schlechtem Schlaf. Es bestand kein Zusammenhang zwischen der Verkehrslärmbelastung, ausgedrückt als $L_{22-6}$, und schlechtem Schlaf. Jedoch zeigte sich ein Trend für die Ausrichtung des Schlafzimmerfensters zu einer ruhigen Fassade und ein signifikanter Synergieeffekt zwischen der Schlafzimmerfensterausrichtung und Job Strain. Schlussfolgerungen: Im Gegensatz zur Schlafzimmerfensterausrichtung erscheint der modellierte nächtliche Straßenverkehrslärmpegel nicht als geeigneter Prädiktor für subjektiv schlechten Schlaf. Zukünftig sollte die Arbeitsbelastung und mögliche Synergieeffekte kontrolliert werden, um 
eine Über- oder Unterschätzung des Einflusses der Lärmbelastung zu vermeiden.

Schlüsselwörter: Verkehrslärm, Arbeitsbelastung, Schlechter Schlaf, Ruhige Fassade, Befragung

15

\section{Zentrales Schlafapnoe-Syndrom als Leitsymptom einer lebensbedrohlichen intrakraniellen Drucksteigerung bei Lhermitte-Duclos-Syndrom}

${ }^{*}$ P. Kutz' , B. Schlüter', M. Koch-Hogrebe ${ }^{2}$, A. Wegener-Panzer ${ }^{3}$, C. Roll ${ }^{1}$

'Vestische Kinder-Jugendklinik Datteln, Universität Witten/Herdecke, Abteilung für Neonatologie, Pädiatrische Intensivmedizin, Schlafmedizin, Datteln, Deutschland; ${ }^{2}$ Vestische Kinder- und Jugendklinik Datteln, Neuropädiatrie, Datteln, Deutschland; ${ }^{3}$ Vestische Kinder- und Jugendklinik Datteln, Universität Witten/Herdecke, Abteilung für Kinderradiologie, Sonographie und Magnetresonanztomographie, Datteln, Deutschland

Fragestellung: Tagesmüdigkeit ist ein häufiges Symptom bei Jugendlichen, eine dringende Indikation zu einer polysomnographischen Untersuchung ergibt sich bei zusätzlichen anamnestischen Hinweisen für eine nächtliche Atemstörung. Im Schlaflabor ist eine Differenzierung zwischen den häufigeren obstruktiven und sehr seltenen zentralen Schlafapnoen möglich. Letztere können Anzeichen einer lebensbedrohlichen intrakraniellen Drucksteigerung sein.

Patienten und Methoden: Eine 15-jährige Patientin wird wegen zunehmender Tagesmüdigkeit mit Notwendigkeit eines Mittagschlafes nach der Schule vorgestellt. Die Eltern berichten, dass sie im Rahmen eines gemeinsamen Urlaubs eine erschwerte nächtliche Atmung mit tiefen Seufzern beobachtet haben. Weitere neurologische Symptome werden verneint, ein auswärtiges EEG sei unauffällig gewesen. Eine MRT im Kleinkindalter wurde bis auf eine Kleinhirnasymmetrie als unauffällig beschrieben. Bei der ansonsten asymptomatischen Patientin wird trotz Corona-Beschränkungen eine stationäre Polysomnographie im Schlaflabor durchgeführt. Ergebnisse: Hier zeigt sich ein schweres zentrales Schlafapnoe-Syndrom, Apnoe-Hypopnoe-Index 94,6 n/h, Desaturierungs-Index 112,2 n/h, mittlere Apnoedauer $24 \mathrm{sec}$, längste Apnoedauer $35,5 \mathrm{sec}$, minimale Sättigung: $74 \%$. Die notfallmäßig durchgeführte MRT zeigt ein raumforderndes Gangliozytom der linken Kleinhirnhemisphäre und des Vermis cerebelli (Lhermitte-Duclos-Syndrom; später histologisch bestätigt) mit beginnender Einklemmung auf Höhe des Foramen magnum und konsekutiv supratentoriellem Hydrozephalus internus occlusus sowie eine Syringomyelie in Höhe HWK 4. Die Patientin wird passager mit einer Atemunterstützung versorgt und einer neurochirurgischen Therapie zugeführt. Das Lhermitte-Duclos-Syndrom ist eine seltene harmatomatöse Erkrankung einer oder beider Kleinhirnhemisphären, die mit Mutationen im Tumor-SuppressorGen PTEN in Zusammenhang gebracht wird.

Schlussfolgerungen: Bei Tagesmüdigkeit sollte auch bei ansonsten asymptomatischen Jugendlichen gezielt nach nächtlichen Atemauffälligkeiten, nicht nur nach Schnarchen, gefahndet werden. Wird ein zentrales Schlafapnoe-Syndrom diagnostiziert, ist eine zeitnahe zerebrale Bildgebung indiziert.

Schlüsselwörter: Zentrales Schlafapnoe Syndrom, Lhermitte-Duclos-Syndrom, Polysomnographie, Intrakranielle Drucksteigerung, Gangliozytom

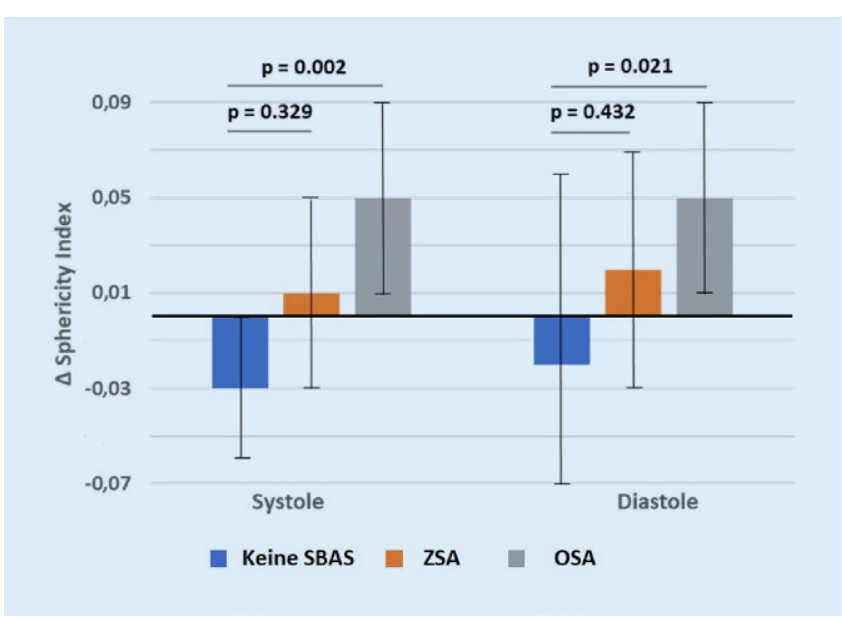

Abb. 1| $16 \Delta$ Balkendiagramm des Sphericity Index von Baseline zu 12-Wochen-Follow-Up in Systole und Diastole. ZSA zentrale Schalafapnoe, OSA obstruktive Schalafapnoe

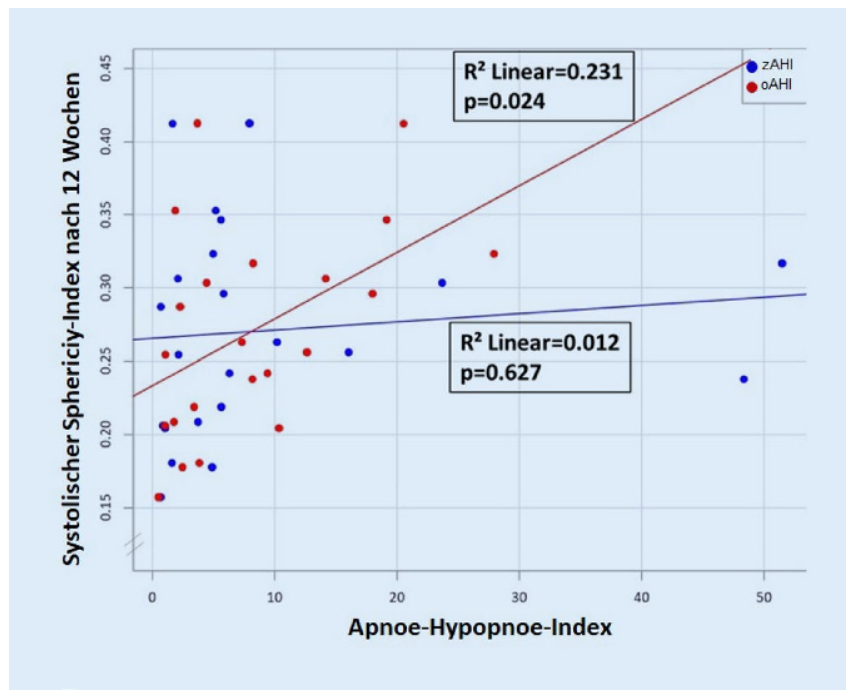

Abb. 2| 16 \ Systolischer Sphericty-Index 12 Wochen nach akutem Myokardinfarkt in Abhängigkeit des Schweregrades der sclafbezogenen Atmungsstörung, unterteilt in obstruktiver Apnoe-Hypopnoe-Index (oAHI) und zentraler Apnoe-Hypopnoe-Index (zAHI)

\section{6}

Die obstruktive Schlafapnoe, aber nicht die zentrale Schlafapnoe ist mit linksventrikulären Remodelling nach akutem Myokardinfarkt assoziiert

${ }^{*}$ C. Fisser', K. Götz', A. Hetzenecker ${ }^{2}$, K. Debl', F. Zeman ${ }^{3}$, O. Hamer', F. Poschenrieder', C. Fellner', S. Stadler', L. Maier', M. Pfeifer', S. Buchner', M. Arzt ${ }^{1}$

'Universitätsklinikum Regensburg, Klinik und Poliklinik für Innere Medizin II, Regensburg, Deutschland; ${ }^{2}$ Krankenhaus Donaustauf, Abteilung für Pneumologie, Donaustauf, Deutschland; ${ }^{3}$ Universitätsklinikum Regensburg, Zentrum für Klinische Studien, Regensburg, Deutschland; ${ }^{4}$ Universitätsklinikum Regensburg, Abteilung für Radiologie, Regensburg, Deutschland; ${ }^{5}$ Krankenhaus Cham, Abteilung für Innere Medizin, Cham, Deutschland

Fragestellung: Die obstruktive Schlafapnoe (OSA) erhöht auf Grund negativer intrathorakaler Druckunterschiede den linksventrikulären transmuralen Druck stärker als die zentrale Schlafapnoe (ZSA). Wir untersuchten 
die Hypothese, dass der Schweregrad der OSA, im Gegensatz zur ZSA, bei Patienten nach akutem Myokardinfarkt mit dem sphärischen linksventrikulären Remodelling assoziiert ist.

Patienten und Methoden: Diese Subanalyse einer prospektiven Observationsstudie untersuchte 24 Patienten mit akutem Herzinfarkt, bei denen eine primäre Koronarintervention erfolgte. Das sphärische linksventrikuläre Remodelling wurde mittels des Sphericity-Index in der kardialen Magnetresonanztomographie 3-5 Tage und 12 Wochen nach akutem Myokardinfarkt analysiert. Die OSA und die ZSA (Apnoe-Hypopnoe-Index [AHI] $\geq 5 / h$ ) wurden mittels Polysomnographie diagnostiziert.

Ergebnisse: Im Vergleich zu Patienten ohne schlafbezogene Atmungsstörungen (SBAS) und zu ZSA Patienten, präsentierten OSA Patienten innerhalb von 12 Wochen nach akutem Myokardinfarkt einen signifikanten Anstieg des systolischen Sphericity-Index (OSA vs. ZSA vs. keine SBAS: $0,05 \pm 0,04$ vs. $0,01 \pm 0,04$ vs. $-0,03 \pm 0,03, p=0,002$, $\bullet$ Abb. 1$)$. Im Gegensatz zur ZSA, war der Schweregrad der OSA mit einem Anstieg des systolischen Sphericity-Index assoziiert (• Abb. 2), auch nach Berücksichtigung von TIMI-Fluss vor Koronarintervention, Infarktgröße, Pain-to-ballontime und systolischem Blutdruck (OSA: B [95\%CI] 0,443 [0,021; 0,816], $p=0,040$; ZSA: $0,193[-0,134 ; 0,300], p=0,385)$.

Schlussfolgerung: Im Gegensatz zur ZSA und keiner SBAS, ist die OSA mit dem sphärischen kardialen linksventrikulären Remodelling innerhalb von 12 Wochen nach akutem Herzinfarkt assoziiert. Die Daten lassen vermuten, dass OSA-bezogene negative intrathorakale Druckunterschiede zu diesem Remodelling nach akutem Myokardinfarkt beitragen.

Schlüsselwörter: Obstruktive Schlafapnoe, Zentrale Schlafapnoe, Kardiales Remodelling, Myokardinfarkt, Kardio-MRT

\section{8}

\section{Limitations of unobtrusive detection of sleep disordered breathing using heart rate variability}

R. Cucu', *F. Braun', J. Van Zaen', P. Renevey', A. Lemkaddem', K. De Jaegere², C. M. Horvath', , C. Roth', A. K. Brill', M. Lemay', S. R. Ott ${ }^{2,4}$

${ }^{1}$ Centre Suisse d'Electronique et de Microtechnique (CSEM), Systems Division, Neuchâtel, Switzerland; ${ }^{2}$ University Hospital (Inselspital) and University of Bern, Department of Pulmonary Medicine, Bern, Switzerland ${ }^{3}$ University Hospital (Inselspital) and University of Bern, Department of Neurology (Sleep-Wake-Epilepsy Centre), Bern, Switzerland; ${ }^{4}$ St. Claraspital, Department of Pneumology, Basel, Switzerland

Objectives: Sleep-disordered breathing (SDB) has a high prevalence in the Swiss population ( $83.8 \%$ in men and $60.8 \%$ in women) [1]. The currently used diagnostics with multichannel sleep studies is often cumbersome and more unobtrusive methods to diagnose SDB are desirable. Single-lead ECG has been investigated for the detection of SDB $[2,3]$. With the emergence of optical heart rate monitoring (PPG) via wrist-worn devices, we investigated whether ECG-based algorithms could be translated to PPG signals for the detection of SDB.

Methods: For the detection of SDB events, we used machine learning algorithms via heart rate variability (HRV). Initially, various algorithms were trained and tested on ECG-derived HRV from 1640 subjects ( 567 without SDB, 525 mild, 310 moderate, 238 severe SDB) from a public dataset of the MESA study [4]. Thereafter, the best performing algorithm was evaluated on 66 subjects ( 35 without SDB, 18 mild, 11 moderate, 2 severe SDB) from a clinical study (NCT03823105) at the Inselspital (Bern, Switzerland). Finally, we used the same algorithm to detect SDB events via HRV obtained from a wrist-worn PPG device from the same subjects.

Results: On the first dataset (MESA) we obtained the following performance using ECG-derived HRV for the detection of SDB events: accuracy $68 \%$, sensitivity $76 \%$, specificity $67 \%$, and precision $21 \%$. On the second dataset (Inselspital) we obtained: accuracy $65 \%$, sensitivity $70 \%$, specificity $65 \%$, and precision $12 \%$. Using PPG-derived HRV from the second dataset resulted in: accuracy of $65 \%$, sensitivity $72 \%$, specificity $64 \%$, and precision $12 \%$.

Conclusions: Compared to previous studies $[3,5]$, our algorithm was trained on a much larger dataset while the resulting performance remains comparable to both ECG- [3] and PPG-based studies [5]. Therefore, we conclude that HRV features alone are not sufficient to reliably detect SDB events. Future work will focus on adding features related to oxygen saturation [6] and PPG-derived respiration [7] to improve SDB diagnostics using a wrist-worn device.

Keywords: Sleep disordered breathing, Heart rate variability, Machine learning, ECG, Wearables

\section{References \\ 1. Heinzer R et al (2015) Lancet Respir Med 3(4):310-318 \\ 2. Stein PK et al (2012) sleep Med Rev 16:47-66 \\ 3. Baty F et al (2020) Sensors $20: 286$ \\ 4. Zhang G-Q et al (2018) J Am Med Inform Assoc 25:1351-1358 \\ 5. Jayawardhana $\mathrm{M}$ et al (2017pp) in. embc:121-124 \\ 6. F. Braun et al. in EMBC 2020, IEEE (2020), im Druck. \\ 7. P. Renevey et al. in EMBC 2018, IEEE (2018), pp. 2861-2864.}

\section{Kurzvorträge}

\section{KV 1 \\ Koffein-sensitive Personen profitieren während chronischem Schlafentzug für zwei Tage von den leistungssteigernden Wirkungen des Kaffees auf die Vigilanz}

${ }^{*}$ E. M. Elmenhorst ${ }^{1}$, D. Elmenhorst ${ }^{2}$, D. Lange ${ }^{1}$, D. Baur ${ }^{3}$, J. Fronczek-Poncelet ${ }^{2}$, E. Hennecke', A. Bauer ${ }^{2}$, H. P. Landolt ${ }^{3}$, D. Aeschbach

'Deutsches Zentrum für Luft- und Raumfahrt, Schlaf und Humanfaktoren, Köln, Deutschland; ${ }^{2}$ Forschungszentrum Jülich, INM-2, Jülich, Deutschland; ${ }^{3}$ Universität Zürich, Institut für Pharmakologie \& Toxikologie, Zürich, Schweiz

Fragestellung: Koffein wird häufig verwendet, um die negativen Wirkungen von Schlafmangel auf die kognitive Leistung zu mildern. Da es bislang wenig wissenschaftliche Erkenntnis zur Frage gibt, für wie lange Koffein die kognitive Leistung bei chronischem Schlafmangel aufrechterhalten kann, untersuchten wir die Wirksamkeit von handelsüblichem Kaffee bei Personen mit genetisch-bedingt hoher Koffein-Sensitivität.

Patienten und Methoden: Im Schlaflabor wurden 71 gesunde Probanden (Alter 20-40 Jahre) untersucht. Das sequentielle Design begann mit einer Adaptations- und zwei Basisnächten mit je 8-stündiger Bettzeit (TIB), an die sich 6 weitere Tage anschlossen, die in 4 Gruppen variiert wurden. Die Kontrollgruppe ( $n=15,5$ Frauen (F)) hatte immer 8 h TIB. Die Restriktionsgruppe $(n=21,9 \mathrm{~F})$ verbrachte 5 Nächte mit $5 \mathrm{~h}$ TIB (experimentelle Studientage E1-E5), denen eine Erholungsnacht (Studientag Rec) mit $8 \mathrm{~h}$ TIB folgte. Die Koffeingruppe ( $n=19,8$ F) hatte dieselben Schlafzeiten wie die Restriktionsgruppe, erhielt aber standardisiert 600 g Kaffee ( 300 mg Koffein) pro Tag während E1-E4 und $400 \mathrm{~g}$ Kaffee ( $200 \mathrm{mg}$ Koffein) an E5. Die entkoffeinierte Gruppe ( $n=16,7 \mathrm{~F})$ durchlief dasselbe Design wie die Koffeingruppe, erhielt jedoch entkoffeinierten Kaffee. Für die beiden Kaffee-Gruppen wurden Träger des C/C-Allels des Adenosin-A2A-Rezeptors (ADORA c.1976) selektiert.

Ergebnisse: Die Gruppen wurden mittels einer gemischten ANOVA mit Bonferroni-Korrektur $(\alpha<0,016)$ hinsichtlich ihrer Verschlechterung in der Tagesleistung (Differenzen zum 2. Basistag in der Reaktionsgeschwindigkeit (1/Reaktionszeit) zum Zeitpunkt 2, 5, 11 und $15 \mathrm{~h}$ wach) in einem 10-minütigen Psychomotorischen Vigilanztest verglichen. Die Restriktionsgruppe war langsamer als die Kontrollgruppe von E1-E5 $(p<0,009)$, aber nicht während $\operatorname{Rec}(p=0,103)$. Die Koffeingruppe war schneller als die entkoffeinierte Gruppe an E1 $(p=0,014)$ und auch schneller als die Restriktionsgruppe während E1 und E2 $(p<0,002)$. Von E3 bis Rec war die Koffeingruppe jedoch langsamer als die Kontrollgruppe $(p<0,014)$. Die entkoffeinierte Gruppe reagierte schneller als die Restriktionsgruppe zum Zeitpunkt $11 \mathrm{~h}$ wach an E1 und E2. Sie war jedoch langsamer als die Kontrollgruppe von E3-E5 $(p<0,006)$, aber nicht während $\operatorname{Rec}(p=0,3710)$. Schlussfolgerungen: Bei Koffein-sensitiven Personen vermochten 300 mg Koffein die kognitive Leistung für zwei Tage auf einem ausge- 
Hier steht eine Anzeige.

\section{算 Springer}


schlafenen Niveau zu halten, führten jedoch zu einer verzögerten Erholung. Auch entkoffeinierter Kaffee entfaltete kurzfristig positive Wirkungen.

Schlüsselwörter: Schlafrestriktion, Koffein, Kognitive Leistung, Adenosin, Erholung

\section{KV 2}

\section{Erweitertes neuronales Netzwerk in den für den Schlaf-Wach- Rhythmus verantwortlichen Hirnzentren}

*F. M. Werner', R. Covenas ${ }^{2}$

${ }^{1}$ Euro Akademie Pößneck, HBFS für Altenpflege, Pößneck, Deutschland; ${ }^{2}$ Institut für Neurowissenschaften von Kastillien und León, Labor 14, Salamanca, Spanien

Fragestellung: In den für Schlaf-Wach-Rhythmus verantwortlichen Zentren stellt sich die Frage, welche Neurotransmitter, Neuropeptide und spezifische Rezeptoren beeinflusst werden können, um eine schlafanstoßende Wirkung zu erzielen. Die bisher publizierten neuronalen Netzwerke werden mit neueren Ergebnisse aktualisiert und erweitert.

Patienten und Methoden: Ein neuronales Netzwerk im Hirnstamm, in dem lateralen Hypothalamus, der Area tegmentalis ventralis und dem präfrontalen Kortex werden dargestellt. Eine Einflussnahme bestimmter Neurotransmitter und Neuropeptide und derer spezifischer Rezeptoren werden abgeleitet, um eine hypnotische Wirkung zu erzielen.

Ergebnisse: Noradrenerge Neurone aus dem Locus coeruleus aktivieren über alpha1-Rezeptoren glutamaterge Neurons, die präsynaptisch serotonergie Neurone aus den dorsalen Raphé-Kernen hemmen. Die serotonergen Neurone aktivieren über 5-HT1A-Rezeptoren GABAerge Neurons, die über nordrenerge Neurons hemmen. Noradrenerge Neurone sind über GABAerge und glutamaterge Neurons mit muscarinergen cholinergen Neurons aus dem mesopontinen Tegmentum in Wechselwirkung. Letztere Neurone sind mit histaminergen Neuronen in Interaktion. Dabei haben H3-antagonistische Medikamente und OX2-Rezeptor-Antagonisten ebenfalls einen hypnotischen Effekt. Im mesolimbischen System interagieren D2-dopaminerge und 5-HT2A-serotonerge Neurons miteinander, wobei dopaminerge Neurone eine aktivierenden und serotonerge Neurone einen beruhigenden Effekt haben. Im präfrontalen Kortex, haben $\mathrm{D} 2$-dopaminerge und muskarinerge cholinerge Neurone eine Interaktion miteinander.

Schlussfolgerungen: Die neuronalen Netzwerke, die aus experimentellen Befunden zur Pharmakodynamik von Agonisten und Antagonisten an spezifischen Rezeptoren und zu Schnittstellen zwischen einzelnen Neurotransmittern und Neuropeptiden abgeleitet sind, sollten weiter optimiert werden. Es ist wichtig, neuere Hypnotika in klinischen Studien zu untersuchen.

Schlüsselwörter: Schlaf-Wach-Rhythmus, Dopamin, Serotonin, Area tegmentalis ventralis, Hypnotika

\section{KV 3}

\section{Aufwandsbegrenztes maschinelles Lernen zur Bestimmung von Schlafstadien}

${ }^{*}$ R. Seepold ${ }^{1,2}$, K. Stuburić', M. Gaiduk ${ }^{1,3}$, N. Martinez Madrid ${ }^{2,4}$, T. Penzel ${ }^{5}$ ${ }^{1}$ HTWG Konstanz, Konstanz, Deutschland; ${ }^{2}$ I. M. Sechenov First Moscow State Medical University, Moskau, Russische Föderation; ${ }^{3}$ University of Seville, Sevilla, Spanien; ${ }^{4}$ Reutlingen University, Reutlingen, Deutschland; ${ }^{5}$ Charité - Universitätsmedizin Berlin, Interdisziplinäres Schlafmedizinisches Zentrum, Berlin, Deutschland

Fragestellung: In diesem Beitrag wird eine Methode des maschinellen Lernens entwickelt, die die Schlafstadienerkennung untersucht. Übliche Methoden der Schlafanalyse basieren auf der Polysomnographie (PSG). Der präsentierte Ansatz basiert auf Signalen, die ausschließlich nicht-invasiv in einer häuslichen Umgebung gemessen werden können. Bewegungs-, Herzschlags- und Atmungssignale können vergleichsweise leicht erfasst werden aber die Erkennung der Schlafstadien ist dadurch erschwert. Die

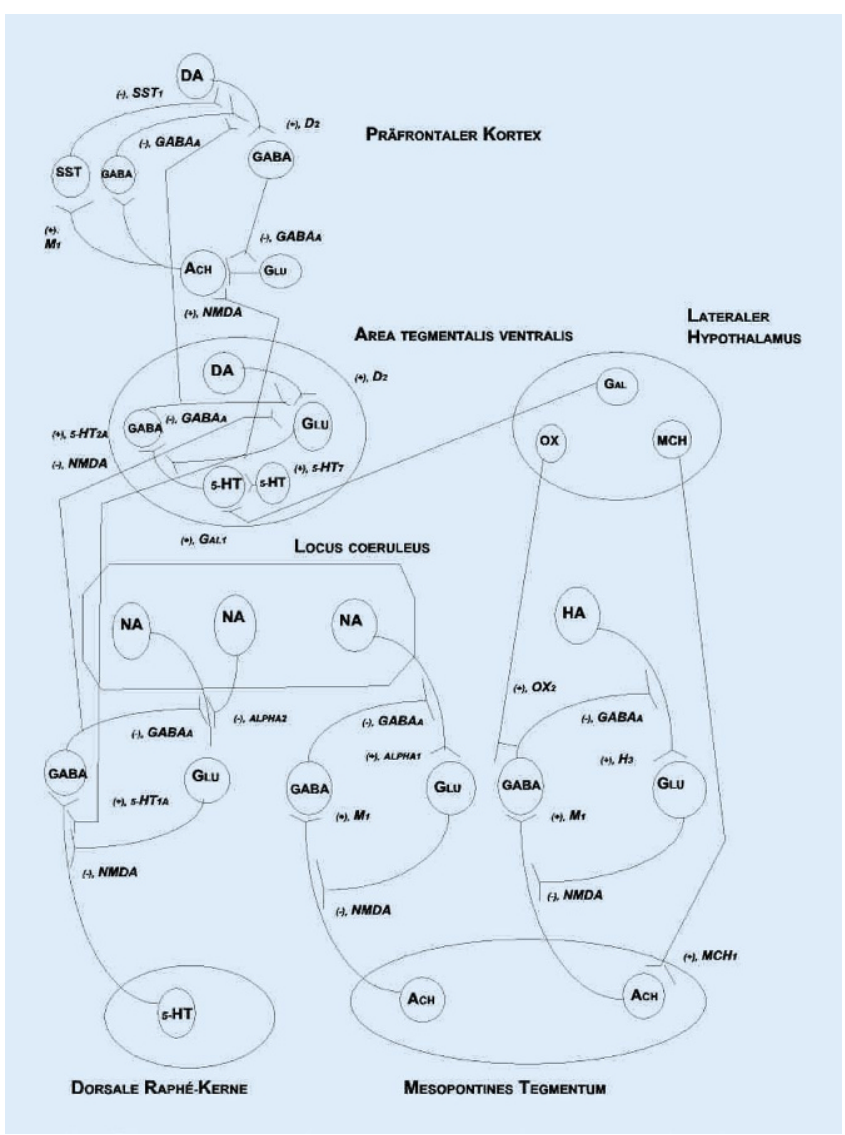

Abb. 1 | KV 2 \ Herzzentren bei der Regulation des Schlaf-Wach-Rhytmus

Signale werden als Zeitreihenfolge strukturiert und in Epochen überführt. Die Leistungsfähigkeit von maschinellem Lernen wird der Polysomnographie gegenübergestellt und bewertet.

Fragestellung: Zwei neuronale Netze wurden getestet: Ein konvolutionäres Netz $(\mathrm{CNN})$ zeichnet sich im Vergleich zu anderen durch geringere Rechenkosten aus und es eignet sich besonders zur Merkmalsextraktion. Gewählt wurden Faltungsschichten mit einer Kerngröße von 80, 40 und 10 sowie zwei vollständig verbundene Schichten, eine mit 100 Neuronen und die andere gleich der Anzahl der Zielklassen.

Das zweite Netzwerk ist ein sog. langes Kurzzeitgedächtnis (LSTM). Jede Klassifizierung einer Stichprobe steht in Relation zu der Vorhergehenden. Da Schlaf einen zyklischen Verlauf hat, erscheint dies sinnvoll. LSTM ist rechenintensiver und es wurde auf 64 Einheiten, gefolgt von drei vollständig verbundenen Schichten begrenzt. Die verwendeten PSG-Daten wurden durch die Charité bereitgestellt. Das Durchschnittsalter der gesunden Patienten lag bei 38,5 Jahren (durchschnittlicher BMI 24,4). Mit 18 Trainingsdaten wurden 5 Klassifizierungen durchgeführt.

Ergebnisse: Für jedes Modell und für jede Klassifikation erreichte die Lerngenauigkeit ca. $80 \%$. Die zunehmende Anzahl von Schichten führte zu einer Erhöhung der Genauigkeit und ggf. auch einer Überanpassung. Um eine Überanpassung zu verhindern, wurden weniger vollständig verbundene Schichten eingefügt. Beim Vergleich der Modelle liefert das CNN bessere Ergebnisse. Dies bedeutet, dass LSTM nicht durch die bisherige Klassifikation profitierte, obwohl das aktuelle Schlafstadium aus den vorhergehenden Stufen relevante und nützliche Informationen ziehen könnte. Der Berechnungsaufwand bei $\mathrm{CNN}$ ist erwartungsgemäß viel geringer als bei LSTM. Die besten Endergebnisse lagen bei ca. $40 \%$ Genauigkeit bei der Klassifizierung in 4 Klassen $\left(\mathrm{F}_{1}\right.$ Score $\left.44 \%\right)$ und bei ca. $55 \%$ bei der Klassifizierung in 3 Klassen ( $F_{1}$ Score $37 \%$ ).

Schlussfolgerungen: Die Erkennung von Schlafstadien wurde als Problem der Zeitreihenklassifikation interpretiert. Es gibt viel Raum für Verbesse- 
rungen und zukünftige Arbeiten, sowohl bei der Modellierung als auch bei der Vorverarbeitung. Eine der Möglichkeiten ist die Datenanreicherung. Durch Datenanreicherung können mehr künstliche Daten generiert werden, die den Lernprozess verbessern können. Eine weitere mögliche Verbesserung ist das Transfer-Lernen, dass bereits für die Klassifizierung des EKG-Signalen eingesetzt wird und dort befriedigende Ergebnisse zeigt. Eine dritte Möglichkeit besteht darin, das Netzwerk zu trennen und beispielsweise drei unabhängige Zweige zu trainieren, und zwar für jedes Signal einzeln. Über eine Ausgabeschicht könnte die Klassifizierung dann wieder zusammengefügt werden.

Schlüsselwörter: Schlafstadien, Maschinelles Lernen, Neuronale Netze, Zeitreihenklassifikation, PSG

\section{KV 4}

\section{Cortisol, Cortison und Dehydroepiandrosteron moderieren den Zusammenhang zwischen Schlafqualität und Burnout}

${ }^{*}$ N. Rothe ${ }^{1}$, K. Schmelzer', S. Vogel', M. Penz ${ }^{1,2}$, M. K. Wekenborg', C. Kirschbaum ${ }^{1}$, A. Walther ${ }^{1,3}$

'Technische Universität Dresden, Lehrstuhl für Biopsychologie, Dresden, Deutschland; ${ }^{2}$ Universitätsklinikum Carl Gustav Carus an der Technischen Universität Dresden, Klinik und Poliklinik für Psychotherapie und Psychosomatik, Dresden, Deutschland; ${ }^{3}$ Universität Zürich, Klinische Psychologie und Psychotherapie, Zürich, Schweiz

Fragestellung: Burnout, definiert als stress-abhängiges Konstrukt, ist mit beeinträchtigter Schlafqualität (SQ) und Schlafstörungen assoziiert (Giorgi et al., 2018; Li et al., 2018). Eine reduzierte SQ ist generell mit psychischem Stress (Rowshan Ravan et al., 2010), als auch mit Veränderungen der stresssensitiven Hypothalamus-Hypophysen-Nebennierenrinden Achsen (HHNA) Aktivität assoziiert (Van Reeth et al., 2000; Rothe et al., 2020). Ungeklärt ist jedoch, ob Hormone der HHNA den Zusammenhang zwischen SQ und dem Burnoutsyndrom beeinflussen.

Patienten und Methoden: Daten zur Untersuchung der Fragestellung stammen aus der vierten Erhebungswelle des Jahres 2018 der Biomarker-Erhebung der Dresdner Burnout Studie (Penz et al., 2018). Insgesamt wurden 462 Proband/innen $\left(M_{\text {Alter }}=43,7 ; 69,0 \%\right.$ weiblich $)$ in die vorliegende Untersuchung eingeschlossen. Allgemeine SQ (inkl. Schlafdauer und Schlafeffizienz) und Burnout Symptomatik wurden durch den Pittsburgh Sleep Quality Index (PSQI) und das Maslach Burnout Inventory - General Survey (MBI-GS) erfasst. Je höher der Gesamtwert des PSQI

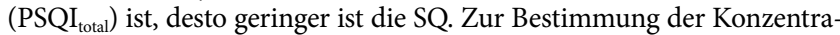
tionen stressassoziierter Hormone (Cortisol, Cortison, Dehydroepiandrosteron [DHEA]), wurden $3 \mathrm{~cm}$ lange Haarproben vom Haaransatz der posterioren vertex Position des Kopfes untersucht und mittels LC-MS/ MS analysiert. Kopfhaar-Hormonkonzentrationen reflektieren langzeitlich eingelagerte Hormonkonzentrationen.

Ergebnisse: Multiple Regressionsanalysen zeigten einen positiven $\mathrm{Zu}-$ sammenhang zwischen PSQI $\mathrm{I}_{\text {total }}$ und Burnout Symptomatik und negative Zusammenhänge zwischen Schlafdauer bzw. Schlafeffizienz und Burnout Symptomatik. Weiterhin wurden Moderationseffekte von Hormonen auf den Zusammenhang zwischen Parametern der SQ und der Burnout Symptomatik identifiziert. Signifikante Interaktionseffekte auf die Burn-

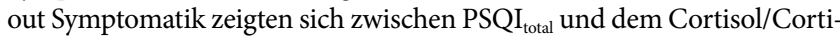
son Ratio, Schlafdauer und DHEA, und Schlafeffizienz und Cortisol bzw. dem Cortisol/Cortison Ratio.

Schlussfolgerungen: Sowohl die allgemeine SQ, als auch die Schlafdauer und Schlafeffizienz sind mit Burnout assoziiert. Stressassoziierte Hormone moderieren den Zusammenhang zwischen SQ und Burnout Symptomatik. Während höhere Cortisol(/Cortison) Konzentrationen eine Verstärkung des Zusammenhangs zeigen, scheint DHEA den Zusammenhang abzumildern. Zukünftige Studien zur Früherkennung oder Prävention von Burnout sollten SQ und endokrine Marker als Risikofaktoren mitberücksichtigen.

Schlüsselwörter: Burnout, Schlafqualität, Cortisol, Cortison, Dehydroepiandrosteron

\section{KV 5}

Wie wach ist die Bundeswehr zu Wasser, Luft und Land? - Erste Ergebnisse eines Fragebogenprojekts

${ }^{*}$ R. Stark' ${ }^{1}$ A. Kashgar ${ }^{1}$, H. Danker-Hopfe ${ }^{2}$, F. Weber ${ }^{3}$, C. Sauter ${ }^{2}$

'Bundeswehr-Krhs Hamburg, Klinik für Neurologie, Hamburg, Deutschland; ${ }^{2}$ Charité Berlin, Kompetenzzentrum Schlafmedizin, Berlin, Deutschland; ${ }^{3}$ Zentrum für Luft- und Raumfahrtmedizin der Luftwaffe, Fürstenfeldbruck, Deutschland

Fragestellung: Schläfrigkeit bei zivilen Pilot*innen, Flugführungspersonal und Wachpersonal auf Schiffen führt zu einem erhöhten Unfallrisiko. Offiziell verfügbare Daten zur Situation in der Bundeswehr fehlen. In diesem Fragebogenprojekt soll daher geklärt werden, ob ein erhöhtes Risiko aufgrund von Tagesschläfrigkeit in der Bundeswehr vorliegen könnte. Patienten und Methoden: Mittels einer anonymen Fragebogenerhebung wurden 1712 Soldat ${ }^{*}$ innen der Marine, der Luftwaffe und einer Kontrollgruppe von Soldat ${ }^{*}$ innen mit regulärem Tagesdienst (KG) kontaktiert. Der Fragebogen setzte sich je nach Einsatzgebiet aus 18 bis 22 Items zur beruflichen Situation und Müdigkeit im Dienstalltag sowie aus der Epworth Schläfrigkeitsskala (ESS), der Fatigue Schweregradskala (FSS) und einem Depressionsfragebogen (PHQ-9) zusammen.

Ergebnisse: Die Gesamtrückläuferquote betrug $n=438$ (25,6\%). Sie variierte je nach Teilstreitkraft: Luftwaffe $14,7 \%(n=163)$, Marine $41,3 \%$ $(n=165)$ und KG $55 \%(n=110)$. Die Soldat ${ }^{*}$ innen der Marine $(46,6 \%)$ zeigten signifikant häufiger auffällige Werte in der ESS als die der Luftwaffe $(31,9 \% ; p=0,009)$, aber nicht im Vergleich zur KG $(34,9 \% ; p=0,061)$. Die Prozentwerte erhöhter FSS-Scores unterschieden sich nicht signifikant $(p=0,174)$ zwischen Marine (47,5\%), Luftwaffe $(53,8 \%)$ und KG (42,2 \%). Schlussfolgerungen: Nahezu die Hälfte der befragten Soldat ${ }^{*}$ innen der Marine wurde als auffallend schläfrig klassifiziert. Die Ergebnisse sind mit einer Studie der US-Marine (Shattuck NL et al. 2016) vergleichbar und am ehesten auf das spezifische Schichtmodell und den Erholungsschlaf beeinträchtigende Faktoren (Lärm, Seegang, Vibrationen) zurückzuführen. Etwa ein Drittel des fliegenden Personals zeigte eine vermehrte Tagesschläfrigkeit und mehr als 50 \% erhöhte Fatigue-Werte. Im Vergleich mit einer zivilen Studie waren diese Werte dennoch niedriger (Reis C et al. 2016), was mit der durchschnittlich kürzeren täglichen Flugdauer mit Rückkehr an den Heimatort am Abend zusammenhängen dürfte.

Zusammenfassend zeigte sich in allen drei Teilstreitkräften bei mindestens einem Drittel eine vermehrte Tagesschläfrigkeit und/oder Fatigue, deren Ursachen nachgegangen werden sollte, um ein erhöhtes Unfallrisiko zu vermeiden.

Schlüsselwörter: Tagesschläfrigkeit, Bundeswehr, Epworth Schläfrigkeitsskala, Piloten, Marine

\section{KV 6 \\ Homeoffice in Zeiten von COVID-19. Wie steht es um die Schlafqualität?}

\section{*M. J. Kater', A. Werner' ${ }^{2}$ A. Lohaus' ${ }^{2}$ A. A. Schlarb}

'Universität Bielefeld, Klinische Psychologie und Psychotherapie des Kindes- und Jugendalters, Bielefeld, Deutschland; ${ }^{2}$ Universität Bielefeld, Entwicklungspsychologie und Entwicklungspathologie, Bielefeld, Deutschland

Fragestellung: Die COVID-19 Pandemie führt zu einer Vielzahl an gesellschaftlichen und arbeitsrelevanten Veränderungen. Eine davon ist das Homeoffice. Laut Statista arbeiteten 2018 etwa $39 \%$ der befragten Arbeitnehmer ganz oder teilweise im Homeoffice, nun unter COVID-19 möchten dies 75,5\%. Neben gesundheitlichen Vorteilen birgt das Homeoffice aber auch Risiken wie Konflikte zwischen Arbeit, Familie und Freizeit sowie einer Auflösung der Tagesstrukturen. Das könnte auch den Schlaf beeinträchtigen, da die Regelmäßigkeit des Schlaf-Wach-Rhythmus wichtig für eine gute Schlafqualität ist. Daher stellt sich die Frage, ob das Arbeiten im Homeoffice aufgrund weniger Tagesstruktur und eines unregelmäßigen Schlaf-Wach-Rhythmus mit einer geringeren Schlafqualität einhergeht. 


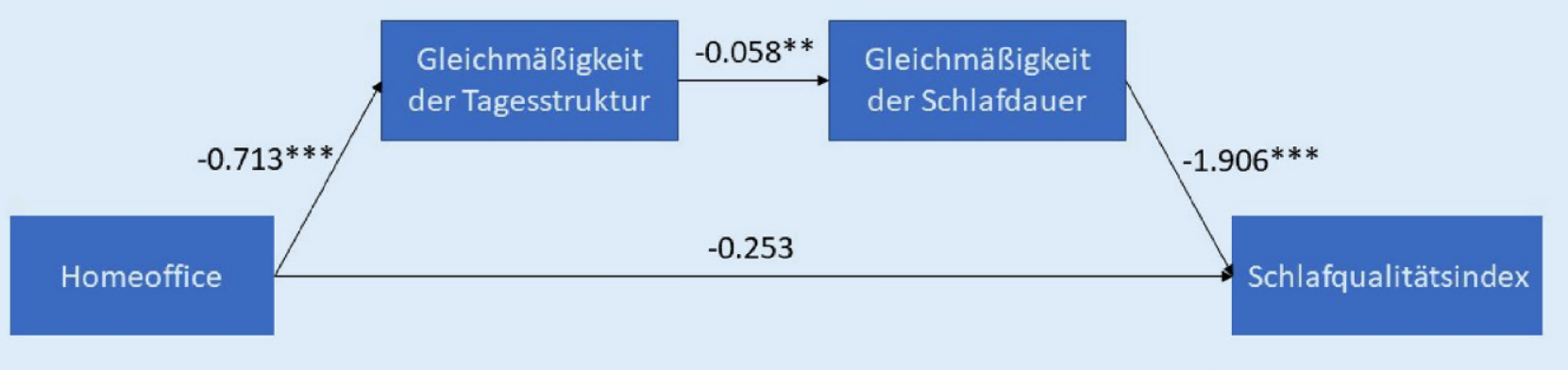

Abb. 1 | KV 6 ॥ Standardisierte Koeffizienten der seriellen Mediationsanalyse. Niedrige Werte in der Gleichmäßigkeit der Tagesstruktur und Schlafdauer deuten auf eine Ungleichmäßigkeit hin sowie ein höherer Schlafqualitätsindex auf eine niedrige Schlafqualität. ${ }^{*} p>.05,{ }^{* *} p>.01,{ }^{* *} p>.001$, ns

Patienten und Methoden: In einer laufenden Online-Befragung wurden die Daten von 438 berufstätigen Personen zwischen 18 und 70 Jahren (127 Männer, xAlter = 36,59 Jahre) erhoben. Davon gaben 213 an, im Homeoffice zu arbeiten. Die Schlafqualität wurde mit dem Pittsburgh Sleep Quality Index erfasst. Zudem wurden die Teilnehmer*innen bezüglich einer Veränderung des Schlaf-Wach-Rhythmus und ihrer Tagesstruktur in der Zeit während COVID-19 und ihrer wahrgenommenen Belastung durch die Einschränkungen von COVID-19 befragt. Mittels serieller Mediationsanalyse wurde untersucht, ob die Verbindung zwischen Homeoffice und Schlafqualitätsindex durch die Variabilität der Tagesstruktur und der Schlafdauer mediiert wird. In dem Modell wird für das Alter, das Geschlecht, die Beeinträchtigung durch COVID-19 kontrolliert.

Ergebnisse: Die beiden seriellen Mediatoren, Tagesstruktur und Schlafdauer, mediieren den Zusammenhang zwischen Homeoffice und Schlafqualitätsindex vollständig, indirekter Effekt $\beta a b c=0,079,95 \%$-KI $[0,008,0,150]$. Dies zeigt, dass Personen im Homeoffice eher eine unregelmäßigere Tagesstruktur aufweisen und dies mit einer höheren Variabilität in der Schlafdauer einhergeht, was wiederum mit einer geringeren Schlafqualität assoziiert ist (s. - Abb. 1).

Schlussfolgerungen: Das Homeoffice stellt somit einen Risikofaktor für einen regelmäßigen Tages- und Schlafrhythmus dar, wodurch auch die Qualität des Schlafes gefährdet werden kann. Als präventive Maßnahme in der COVID-19-Krisensituation sollte eine Empfehlung zur Einhaltung von Tagesstruktur und Work-Life-Balance im Homeoffice verbreitet werden, um den Schlaf der Betroffenen zu verbessern.

Schlüsselwörter: Homeoffice, Schlafqualität, Tagesstruktur, Schlafdauer, COVID-19

\section{KV 7}

\section{Quiet-Sleep Erkennung mit künstlicher Intelligenz bei Säuglingen}

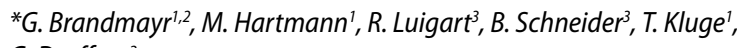
G. Dorffner ${ }^{2}$

'AIT Austrian Institute of Technology $\mathrm{GmbH}$, Center for Health \& Bioresources, Wien, Österreich; ${ }^{2}$ Medizinische Universität Wien, Institut für Artificial Intelligence and Decision Support, Wien, Österreich; ${ }^{3}$ Kinderkrankenhaus St. Marien gGmbH, Zentrum für Kinder- und Jugendmedizin, Landshut, Deutschland

Fragestellung: Die regelmäßige Beobachtung der Schlafarchitektur von Neugeborenen liefert wertvolle, objektive Einblicke in die Gehirnreifung und neurologische Gesundheit. Die visuelle Bewertung von neonatalem EEG in Kombination mit nicht-cerebralen Signalen gilt als klinischer Goldstandard. Die klinische Anwendbarkeit könnte durch Reduktion des Instrumentierungs- und Zeitaufwandes deutlich erleichtert werden. Wir stellen ein auf künstlicher Intelligenz basiertes Verfahren zur Quiet-Sleep Abschätzung vor, das mit nur einem EEG-Kanal auskommt.

Patienten und Methoden: Wir zeigen eine retrospektive Analyse von 27 Säuglingen im Alter von bis zu 6 Monaten, welche im Zentrum für Neuropädiatrie und Schlafmedizin des Kinderkrankenhauses St Marien in Landshut mindestens 1-mal im Schlaflabor untersucht wurden. Die Schlafstadien wurden aus der aufgezeichneten 20-Kanal Polysomnographie gemäß dem Manual von T. Anders (1971) von Somnologen in 30 s langen Epochen bestimmt. Ein „deep convolutional neural net" mit 11 Schichten wurde mit dem frontalen EEG-Kanal F3-A2 trainiert das QuietSleep Schlafstadium zu erkennen. Durch den end-to-end-learning Ansatz werden die Limitierungen von manuell entwickelten Signal-Features überwunden. Die wiederholte, schrittweise Reduktion der zeitlichen Auflösung ermöglicht ein weites, rezeptives Feld von über $20 \mathrm{~s}$ in der letzten Lage des Netzwerks. Die Leistung des Verfahrens wurde mit 27-facher Kreuzvalidierung anhand der Übereinstimmung nach Cohens Kappa bewertet. Ergebnisse: Im epochenweisen Vergleich wurde ein kumulatives Kappa von 0,64 erzielt. Für einzelne Nächte ergibt sich ein medianes Kappa von 0,68 zwischen den Quartilen Q1 =0,54 und Q3 = 0,75. Zwei Nächte wichen mit Kappa 0,14 auffällig ab, und sind nahe am Zufalls-Kappa 0.

Schlussfolgerungen: Der erreichte Kappa Wert von 0,64 entspricht lt. Landis Skala einer „beachtlichen Übereinstimmung“. Verglichen mit der Literatur haben Verfahren, die alle EEG Kanäle verwenden höhere Kappa Werte. Das beste in der Literatur gefundene Verfahren für 1 EEG-Kanal erzielt ein Kappa von 0,60 (Ansari et al., 2020). Die 2 auffälligen Nächte weisen möglicherweise auf fehlerhafte Daten hin. Unter Ausschluss der 2 Nächte ist das vorgestellte Verfahren statistisch signifikant besser (t-Test, $p=0,04$ ).

Die Ergebnisse zeigen, dass bereits ein frontaler EEG Kanal viel Information über das Schlafstadium enthält, und Quiet-Sleep Bestimmung bei Säuglingen mit deutlicher weniger aufwendiger Instrumentierung denkbar ist.

Schlüsselwörter: EEG, Quiet-Sleep, Deep Learning, Säuglinge, Convolutional Neural Net

\section{KV 8}

The impact of treatment of periodic limb movements in sleep on blood pressure

${ }^{*}$ D. Toma', M. Seidel', A. Doevelaar', F. Bauer', F. S. Seibert', B. Rohn', N. Babel ${ }^{1,2}$, N. Büchner ${ }^{3}$, S. Wang' ${ }^{1}$, T. H. Westhoff'

'Universitätsklinikum Marien Hospital Herne, Medizinische Klinik I, Herne, Germany; ${ }^{2}$ Universitätsklinikum Marien Hospital Herne, Centrum für Translationale Medizin, Herne, Germany; ${ }^{3} \mathrm{HELIOS}$-Klinikum Duisburg, Pneumologie, Schlaf- und Beatmungsmedizin, Duisburg, Germany

Objectives: Improving sleep quality in patients with obstructive sleep apnea by positive airway pressure therapy is associated with a decrease of blood pressure (BP). It remains elusive, however, whether treatment of sleep disturbances due to periodic limb movements in sleep (PLMS) affects $\mathrm{BP}$ as well. The present study provides first data on this issue.

Methods: Retrospective study on 114 patients undergoing in-laboratory polysomnography in a German University Hospital. Inclusion criteria were first diagnosis of PLMS with a periodic leg movement index $\geq 15 / \mathrm{h}$ and periodic leg movement arousal index $\geq 5 / \mathrm{h}$ with subsequent initia- 
tion of therapy with dopamine or dopamine agonists. Exclusion criterion was an initiation or change of preexisting positive airway pressure therapy between baseline and follow-up polysomnography. BP and Epworth sleepiness scale (ESS) were assessed at two consecutive in-laboratory polysomnographies. BP was assessed auscultatorily according to Riva-Rocci at admission.

Results: Mean age of the study population was $62.1 \pm 12.1$ years. 90 patients $(78.9 \%)$ suffered from hypertension, $89(78.0 \%)$ had antihypertensive medication. 76 subjects $(66.6 \%)$ had preestablished CPAP therapy. All the patients were diagnosed to have PLMS. 100 patients (87.7\%) were started on dopamine (mean dosage $159.0 \pm 67.8 \mathrm{mg}$ ), 14 patients $(12.2 \%)$ on dopamine agonists after the baseline examination (pramipexole, mean dosage $0.3 \pm 0.1 \mathrm{mg}$, ropinirol $2.5 \pm 1.5 \mathrm{mg}$ ). ESS significantly decreased from baseline to follow-up from 6 (interquartile range (IQR) 3-10.5) to 5 (IQR 3-10, p 0.013). Systolic BP significantly decreased from $132.9 \pm 17.1$ to $127.1 \pm 19.2 \mathrm{mmHg}$ ( $\mathrm{p} 0.004$ ), whereas there was no change of diastolic BP $(75.9 \pm 12.5$ vs. $75.1 \pm 9.2 \mathrm{mmHg}$, p 0.48). The number of antihypertensive drugs remained unchanged with a median of 2 at baseline and follow-up (p 0.27).

Conclusions: Treatment of PLMS by dopamine or dopamine agonists is associated with an improvement of sleep quality and a decrease of systolic but not diastolic BP.

Keywords: Periodic leg movement in sleep, Treatment, Blood pressure, Sleep disturbances, Dopamine

\section{KV 9 \\ Das multidimensionale Dyspnoeprofil (MDP) bei COPD und Schlafapnoe}

*W. Galetke', V. Jansen ${ }^{2}$, A. Tschesche ${ }^{3}$, U. Domanski', M. Schröder', G. Nilius ${ }^{4}$ 'VAMED Klinik Hagen-Ambrock, Pneumologie, Hagen, Deutschland; ${ }^{2}$ Praxis Dr. med V. Jansen, Menden, Deutschland; ${ }^{3}$ Universität Witten-Herdecke, Witten, Deutschland; ${ }^{4} \mathrm{KEM}$ | Kliniken Essen-Mitte, Essen, Deutschland

Fragestellung: Das Multidimensionale Dyspnoe Profil (MDP) ist ein Fragebogen zur multidimensionalen Erfassung des Symptoms Dyspnoe. Dyspnoe gilt als eigenständiger Prädiktor für Mortalität bei älteren Patienten und bei Patienten mit Herzerkrankungen oder chronisch obstruktiver Bronchitis (COPD). Gerade bei der COPD kommen subjektiven Parametern in der Schweregrad-Einteilung eine hohe Bedeutung zu. Luftnot als Folge einer obstruktiven Schlafapnoe ist als Symptom bislang nicht gut in Studien untersucht. Vorstellbar ist hier durchaus ein kausaler Zusammenhang durch Folgeerkrankungen oder durch eine noch am Morgen be-

\begin{tabular}{|l|c|c|c|c|c|c|}
\hline Tabelle 1 & \multicolumn{3}{|c|}{ COPD stabil } & \multicolumn{3}{c|}{ OSA } \\
\cline { 1 - 1 } MDP Skalen (score 0-10): & N & MW & SD & N & MW & SD \\
\hline A1 Atemgefühl & 125 & $\mathbf{2 , 4}$ & 1,9 & 99 & $\mathbf{0 , 6}$ & 1,5 \\
\hline SQ Muskelarbeit / Kraftaufwand & 127 & $\mathbf{1 , 7}$ & 1,8 & 100 & $\mathbf{0 , 4}$ & 1,0 \\
\hline SQ Erstickungsgefühl/Lufthunger & 127 & $\mathbf{1 , 7}$ & 2,3 & 100 & $\mathbf{0 , 4}$ & 1,1 \\
\hline SQ Enge Lungen/Brustkorb & 126 & $\mathbf{1 , 9}$ & 2,3 & 100 & $\mathbf{0 , 6}$ & 1,6 \\
\hline SQ Konzentration auf die Atmung & 126 & $\mathbf{1 , 6}$ & 2,2 & 100 & $\mathbf{0 , 2}$ & 0,8 \\
\hline SQ viel atmen & 127 & $\mathbf{2 , 7}$ & 2,7 & 100 & $\mathbf{0 , 6}$ & 1,6 \\
\hline A2 Deprimiert & 127 & $\mathbf{1 , 4}$ & 2,2 & 100 & $\mathbf{0 , 3}$ & 1,0 \\
\hline A2 Besorgt & 127 & $\mathbf{2 , 3}$ & 2,4 & 100 & $\mathbf{1 , 1}$ & 2,1 \\
\hline A2 Frustriert & 127 & $\mathbf{1 , 6}$ & 2,4 & 100 & $\mathbf{0 , 4}$ & 1,4 \\
\hline A2 Verärgert & 127 & $\mathbf{1 , 7}$ & 2,8 & 100 & $\mathbf{0 , 4}$ & 1,5 \\
\hline A2 Ängstlich & 127 & $\mathbf{2 , 1}$ & 2,7 & 100 & $\mathbf{0 , 7}$ & 1,5 \\
\hline
\end{tabular}

\section{Abb. 1 | KV 9 A}

stehende Hyperkapnie. Die vorliegende Untersuchung ist Teil einer multizentrischen, prospektiven Kohorten-Studie. Ziel ist es die individuelle Beschwerdesymptomatik einer COPD-Patientengruppe zu erfassen und die Aussagefähigkeit des Fragebogens im Vergleich zu Patienten mit einer obstruktiven Schlafapnoe (OSA) als Kontrollgruppe zu validieren.

Fragestellung: Es wurden COPD-Patienten in einer stabilen Phase im Rahmen von elektiven Vorstellungen bei einem niedergelassenen Pneumologen bzw. in einer pneumologischen Fachklinik und OSA-Patienten eines stationären Schlaflabors einmalig mit dem MDP befragt. Der MDP Fragebogen erfasst das Symptom Dyspnoe mit einer Skala zur übergreifenden Quantifizierung (A1 Skala), fünf sensorische Qualitäten (SQ Skalen) und fünf emotionalen Komponenten (A2 Skalen). Die Skalen liefern jeweils Werte von 0-10. Je höher der numerische Wert ist, desto ausgeprägter ist die Komponente.

Ergebnisse: 127 Patienten mit COPD (GOLD II: $N=67$; GOLD III: $N=44$; GOLD IV: $N=16$; weiblich: 54 (42,5\%); Alter: 67,1 $\pm 8,5$; BMI: 26,9 $\pm 5,95$; FEV1: 1,3L $\pm 0,5$ l; mMRC: $1,76 \pm 1,18$ ) wurden verglichen mit 100 OSA Patienten (weiblich: 25 (25\%); Alter: 53,0 $\pm 13,3$; BMI: 33,51 $\pm 7,4$; AHI: 39,6 $\pm 25,4$; mMRC: 0,79 $\pm 0,97)$. Der Unterschied der Scores der A1 Scala zwischen den beiden Kohorten erwies sich als hochsignifikant $(P<0,001)$ Die - Abb. 1 zeigt die Ergebnisse der MDP-Skalen im Überblick.

Schlussfolgerungen: Die Untersuchung konnte zeigen, dass das Symptom Dyspnoe bei COPD Patienten gegenüber einer Gruppe von OSA-Patienten mit erwartungsgemäß geringem Luftnotempfinden mit dem MDP differenziert dargestellt werden kann. Möglicherweise ist der MDP ein geeignetes Instrument, um das Ausmaß des Phänomens Luftnot bei COPD

\section{Hier steht eine Anzeige.}


Patienten besser erfassen zu können. Dazu sind jedoch noch Untersuchungen an weiteren Patientengruppen notwendig.

Schlüsselwörter: Dyspnoe, Luftnotempfinden, OSA, COPD, Fragebogen

\section{KV 10}

\section{A novel algorithm for the estimation of sleep stages based on breathing and movement}

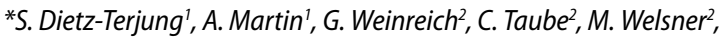 C. Schöbel \\ 'Universitätsmedizin Essen, Ruhrlandklinik, Lehrstuhl für Schlaf- und Telemedizin, Essen, Germany; ${ }^{2}$ Universitätsmedizin Essen, Ruhrlandklinik, Klinik für Pneumologie, Essen, Germany}

Objectives: We tested the diagnostic accuracy of the novel Nox Body Sleep $^{\text {tw }}$ algorithm (Nox medical, Island) for the estimation of sleep stages from polygraphy $(\mathrm{PG})$ sleep recordings based on features extracted from actigraphy and respiratory inductance plethysmography (RIP) belts. Validation was performed against polysomnography (PSG) in a cohort including patients with sleep disordered breathing (SDB).

Methods: Patients received PSG according to clinical routine. The recording was evaluated by the novel algorithm and the results were evaluated by descriptive statistics methods (IBM SPSS Statistics 25.0).

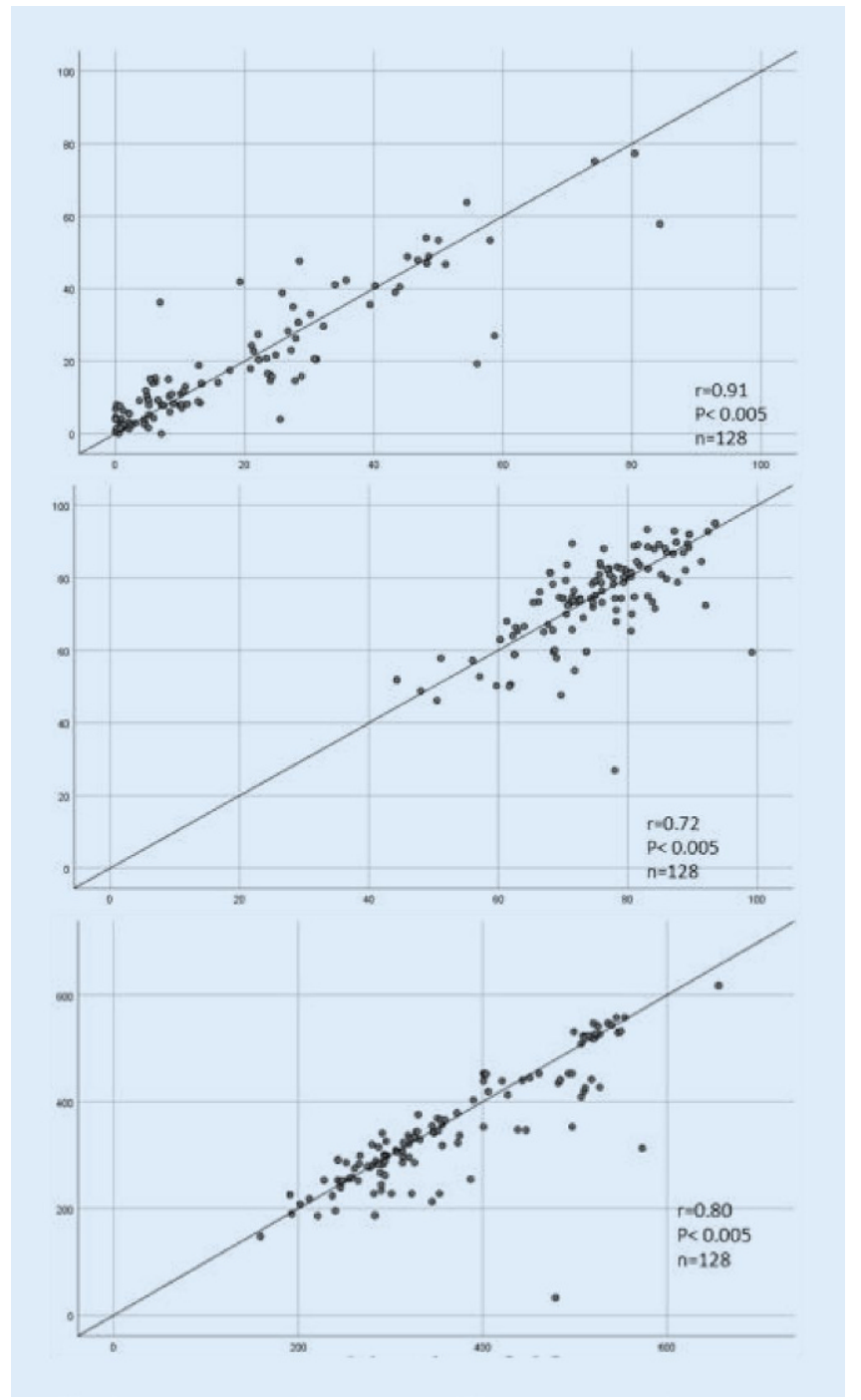

Fig. 1 | KV 10 ॥ Correlation analysis for a AHI, b Sleep Efficiency and c Total Sleep Time
Results: We found a strong Pearson correlation $(\mathrm{r}=0.81)$ and an overestimation of $14 \mathrm{~min}$ for Total Sleep Time. Sleep Efficiency was also valued with a strong Pearson correlation $(\mathrm{r}=0.73)$ and an overestimation of $2.1 \%$. Additionally we found a strong correlation for AHI estimation $(r=0.91)$ and an overestimation of $0.2 / \mathrm{h}$. Wake phases were estimated with a F1 score of 0.62 while REM and Non-REM phases were evaluated with a F1 score of 0.72 and 0.70 , respectively. Additionally, a Cohens kappa of 0.62 were found.

Conclusions: The algorithm shows a strong diagnostic accuracy for the estimation of sleep stages and significant sleep parameters and could therefore used for a more appropriate home-based testing. Further validation on a larger patient collective is necessary to improve the algorithm.

Keywords: Sleep state estimation, Telemedicine, Artificial intelligence, Sleep, Sleep disordered breathing

\section{KV 11}

Towards unobtrusive detection and monitoring of sleep disordered breathing using wearables: validation of watch-based measurement of oxygen saturation, respiration and heart rate

*F. Braun', P. Renevey', P. Theurillat', M. Proença', J. Van Zaen', A. Lemkaddem', D. Ferrario' ', K. De Jaegere', C. M. Horvath ${ }^{2,3}$, C. Roth ${ }^{3}$, A. K. Brill', M. Lemay', S. R. Ott ${ }^{2,4}$

${ }^{1}$ Centre Suisse d'Electronique et de Microtechnique (CSEM), Systems Division, Neuchâtel, Switzerland; ${ }^{2}$ University Hospital (Inselspital) and University of Bern, Department of Pulmonary Medicine, Bern, Switzerland: ${ }^{3}$ University Hospital (Inselspital) and University of Bern, Department of Neurology (Sleep-Wake-Epilepsy Centre), Bern, Switzerlandz; ${ }^{4}$ St. Claraspital, Department of Pneumology, Basel, Switzerland

Objectives: Polysomnography (PSG) or home sleep apnea testing (HSAT) with respiratory polygraphy are the current standard diagnostics for sleep disordered breathing (SDB). These systems require a multitude of sensors, are obtrusive and thus not suited for long-term monitoring. Recent advances in optical heart rate monitoring give promise for wearable devices which are low-cost and might be suitable for the early detection and ambulatory monitoring of SDB. Even though not expected to completely replace PSG or HSAT, such devices could allow for a reliable pre-screening over several consecutive nights in larger populations. We investigated the feasibility of a wearable device to measure oxygen saturation (SpO2), respiratory rate (RR) and cardiac interbeat intervals (IBI).

Methods: We compared a wrist-worn device against PSG on 66 subjects (27 healthy volunteers and 39 patients with sleep disorders) in a clinical study (NCT03823105) at the Inselspital (Bern, Switzerland). In parallel to an overnight PSG, accelerometer and optical heart activity were measured via CSEM's smartwatch device. From the watch-based signals, $\mathrm{SpO} 2$ [1], RR [2], [3] and IBIs [2] were estimated and compared to PSG-derived reference measurements in terms of RMS error (for SpO2) and mean absolute error (for RR and IBI).

Results: Watch-based SpO2 estimates show an RMS error of ARMS $=3.2 \%$ (after the automatic rejection of $15.8 \%$ by our algorithm [4]) which is compliant with the ISO 80601-2-61:2017 standard and FDA guidance for reflectance type sensors. Moreover, RR and IBI were estimated with a subject-wise mean absolute error of $1.55 \pm 1.04$ breaths/min and $6.54 \pm 5.41$ milliseconds.

Conclusions: The present results show promising performances for watchbased estimation of SpO2, RR and IBIs during sleep. Limited by a rather small dataset, this approach should be tested in a larger population, especially on patients with moderate to severe SDB. Future work will focus on combining SpO2, RR and IBI estimates to provide a low-cost solution for the early detection and long-term ambulatory monitoring of SDB.

Keywords: Sleep disordered breathing, Wearables, Oxygen saturation, Respiratory rate, Heart rate

\section{References}

1. F. Braun et al. in $E M B C 2020$, IEEE (2020), im Druck.

2. P. Renevey et al. in EMBC 2018, IEEE (2018), pp. 2861-2864. 
3. Papini GB et al (2020) Physiol Meas : (in press)

4. M. Proenca et al. in EMBC 2018, IEEE (2018), pp. 1502-1505.

\section{KV 12}

\section{Die akustische Analyse von Schnarchmustern zu Identifikation} von Obstruktionsarten in Abhängigkeit vom Body-Mass-Index

${ }^{*}$ H. Bloutian ${ }^{1}$, C. Janott ${ }^{2}$, C. Bergmann ${ }^{3},{ }^{*}$ W. Hohenhorst ${ }^{1}$

${ }^{1}$ Alfried-Krupp-Krankenhaus, Essen Rüttenscheid, HNO, Essen, Deutschland; ${ }^{2}$ Technische Universität München, Berlin, Deutschland; ${ }^{3}$ HNO Praxis Ruhr, HNO, Essen, Deutschland

Fragestellung: Das obstruktive Schlafapnoe Syndrom (OSAS) ist die häufigste schlafbezogene Atmungsstörung mit erhöhter kardiovaskulärer Morbidität. Ein Hauptsymptom ist die Rhonchopathie. Als gemeinsamer und unabhängiger Risikofaktor, sowohl für kardiovaskuläre Erkrankungen als auch für OSAS, konnte unter anderem die Adipositas identifiziert werden. Zielsetzung der Studie ist die Identifikation spezifischer Schnarchmuster adipöser Patienten zur einfachen Detektierung und gegebenenfalls zur Therapieoptimierung dieser besonders gefährdeten Patientengruppe. Patienten und Methoden: In dieser Studie wurde untersucht, in welchem Zusammenhang der Body-Mass-Index (BMI > 30) mit dem endoskopischen Obstruktionsmechanismus und dem Schnarchmuster steht. Die Daten wurden während einer medikamentös-induzierten Schlafendoskopie (MISE) erhoben. Die Auswertung erfolgte anhand der geläufigen VOTEKlassifikation, als auch nach der geräuschkorrelierten ACLTE-Einteilung. Verglichen wurde mittels Chi-Quadrat Test die Häufigkeit des Vorkommens der jeweiligen Schnarchmuster.

Ergebnisse: Es zeigte sich, dass ein statistisch signifikanter Zusammenhang zwischen einem erhöhten BMI und Schnarchmustern auf uvulovelär Ebene anterior-posterior $(p<0,01)$ und der lateralen Oropharynxwand $(p<0,005)$ besteht. Daneben wird deutlich, dass Patienten mit einem erhöhten BMI eine Tendenz zu einem geringeren Apnoe-Hypopnoe-Index (AHI) nach bereits erfolgter Tonsillektomie aufweisen. Im Weiteren zeigt sich unabhängig vom BMI keine Tendenz zu einem spezifischen Schnarchmuster bei bereits tonsillektomierten Patienten.

Schlussfolgerungen: Adipösen Patienten sollte eine Gewichtsreduktion als entscheidender Bestandteil der Therapie empfohlen werden. Basierend auf den vorliegenden Daten sollte bei einem unzureichenden konservativen Therapieansprechen die Tonsillektomie mit gegebenenfalls Uvulopalatopharyngoplastik erwogen werden, da vor allem eine laterale Obstruktionskomponente anzunehmen ist.

Schlüsselwörter: OSAS, VOTE, ACLTE, BMI, Adipositas

\section{KV 13}

\section{Association between habitual physical activity (HPA) and sleep quality in patients with cystic fibrosis}

*S. Dietz-Terjung', W. Gruber' ${ }^{2}$ S. Sutharsan ${ }^{3}$, C. Taube ${ }^{3}$, M. Olivier², U. Mellies', C. Koerner-Rettberg ${ }^{4}$, S. Dillenhöfer', F. Stehling ${ }^{2}$, M. Welsner ${ }^{3}$

'Universitätsmedizin Essen, Ruhrlandklinik, Lehrstuhl für Schlaf- und Telemedizin, Essen, Germany; ${ }^{2}$ Pediatric Pulmonology and Sleep Medicine, Cystic Fibrosis Center, Children's Hospital, University of Duisburg-Essen, Essen, Germany; ${ }^{3}$ Universitätsmedizin Essen, Ruhrlandklinik, Klinik für Pneumologie, Essen, Germany; ${ }^{4}$ Department of Pediatric Pneumology, University Children's Hospital, Ruhr University Bochum, Bochum, Germany

Objectives: Sleep disturbances and poor sleep quality are known to be present in patients with CF. Regular physical activity plays an important role in the treatment of CF patients due to its positive influence on progression of disease and quality of life. The aim of this work is to create a home-based sleep and activity profile and to investigate the influence of habitual physical activity (HPA) on sleep quality of children, adolescents and adults with CF.

Methods: 109 CF patients (64 male, mean age $22.7 \pm 12.0$ years; mean ppFEV1 63.0 \pm 26.7 ) were equipped with an actigraph for home-based collection of activity and sleep data for a total of 4 weeks.
Results: CF patients under the age of 18 show the best sleep efficiency $(92 \pm 3 \%)$, the longest TIB $(545 \pm 71 \mathrm{~min})$ and TST $(504 \pm 72 \mathrm{~min})$ and the lowest WASO $(39 \pm 15 \mathrm{~min})$. With increasing age there is a decrease in SE $(p<0.01)$, TST $(p<0.01)$ and TIB $(p<0.01)$ and an increase in WASO ( $p>0.05)$. In terms of lung function, CF patients with FEV1 values $>70 \%$ pred. have the best SE $(92 \pm 3 \%)$, the highest TST ( $490 \pm 72 \mathrm{~min})$ and TIB $(531 \pm 71 \mathrm{~min})$ and the lowest WASO $(39 \pm 13 \mathrm{~min})$. As FEV1 deteriorates, a change in sleeping behavior can be detected. SE $(p<0.05)$, TST $(p<0.05)$ and TIB $(p>0.05)$ decrease, whereas WASO $(p>0.05)$ increases. Interestingly, there is no difference in the number of awakenings between the different age and FEV1 groups (all $p>0.05$ ).

Conclusions: Besides younger age and higher FEV1, daily activity in higher intensities influences sleep of CF patients in a positive way. Patients with poor sleep quality and sleep disturbances possibly benefit from an intensification of physical activity in the home environment.

Keywords: Out of center testing, Cystic fibrosis, Sleep, Activity, Actigraphy

\section{KV 14}

\section{Frequency and duration of apneas during sleep in infants and} children: A meta-analysis

\section{*. Kelmanson ${ }^{1,2}$}

'Institute for Medical Education of the V. A. Almazov National Medical Research Centre, Department of Children's Diseases, St. Petersburg, Russia; ${ }^{2}$ St. Petersburg State Institute for Psychology and Social Work, Department of Clinical Psychology, St. Petersburg, Russia

Objectives: Much interest has been raised in the apneic events during sleep in infants and children. Reference values on the frequency and duration of different types of apneas during sleep in normal subjects are sparse, most of which are from the 1980s and 1990s. As a rule, they cover limited numbers of observations. The study aimed to perform a meta-analysis of the frequency and duration of different types of apneas in infants and children in sleep based on contemporary publications.

\begin{tabular}{|c|c|c|c|c|c|c|c|c|c|c|}
\hline \multirow[t]{3}{*}{ Study } & \multirow[t]{3}{*}{ Year } & \multirow{3}{*}{$\begin{array}{l}\text { Group } \\
\mathrm{N}\end{array}$} & \multicolumn{6}{|c|}{ Apnea indices per 1 hour of total sleep time } & \multirow{3}{*}{$\begin{array}{l}\text { Mean } \\
\text { age, } \\
\text { mo }\end{array}$} & \multirow{3}{*}{$\begin{array}{l}\text { Percen } \\
\text { boys }\end{array}$} \\
\hline & & & \multicolumn{2}{|c|}{$\begin{array}{l}\text { Central } \\
\text { apneas }\end{array}$} & \multicolumn{2}{|c|}{$\begin{array}{l}\text { Obstructive } \\
\text { apneas }\end{array}$} & \multicolumn{2}{|c|}{$\begin{array}{l}\text { Mixed } \\
\text { apneas }\end{array}$} & & \\
\hline & & & Mean & SD & Mean & SD & Mean & SD & & \\
\hline $\begin{array}{l}\text { Brockmann et } \\
\text { al.-1 }\end{array}$ & 2013 & 37 & 5.500 & 10.850 & 0.800 & 1.650 & 0.300 & 0.300 & 1.000 & 59.000 \\
\hline $\begin{array}{l}\text { Brockmann et } \\
\text { al.-2 }\end{array}$ & 2013 & 37 & 4.100 & 6.525 & 0.800 & 0.575 & 0.100 & 0.200 & 3.000 & 59.000 \\
\hline Coh et al. & 2000 & 10 & NA & NA & 0.000 & 0.100 & NA & NA & 72.000 & 30.000 \\
\hline Daftary et al. & 2019 & 30 & 5.400 & 6.200 & 2.300 & 2.500 & 1.200 & 1.500 & 0.630 & 40.000 \\
\hline Kato et al.-1 & 2000 & 63 & NA & NA & 0.300 & 0.725 & 0.100 & 0.400 & 1.500 & 35.000 \\
\hline Kato et al.-2 & 2000 & 521 & NA & NA & 0.200 & 0.450 & 0.100 & 0.670 & 2.500 & 47.000 \\
\hline Kato et al.-3 & 2000 & 238 & NA & NA & 0.200 & 0.117 & 0.100 & 0.150 & 3.500 & 42.000 \\
\hline Kato et al.-4 & 2000 & 137 & NA & NA & 0.200 & 0.283 & 0.100 & 0.083 & 4.500 & 49.600 \\
\hline Kato et al..-5 & 2000 & 64 & NA & NA & 0.100 & 0.250 & 0.100 & 0.075 & 5.500 & 50.000 \\
\hline $\begin{array}{l}\text { Montgomery- } \\
\text { Downs et al.-1 }\end{array}$ & 2013 & 153 & 0.820 & 0.730 & 0.030 & 0.100 & 0.010 & 0.050 & 58.800 & 54.900 \\
\hline $\begin{array}{l}\text { Montgomery- } \\
\text { Downs et al.-2 }\end{array}$ & 2013 & 388 & 0.450 & 0.490 & 0.050 & 0.110 & 0.010 & 0.060 & 81.600 & 51.500 \\
\hline Moss et al. & 2005 & 50 & 1.500 & 1.100 & 0.100 & 0.200 & 0.010 & 0.040 & 121.200 & 52.000 \\
\hline Sanchez et al. & 2006 & 320 & NA & NA & 0.467 & 0.770 & 0.580 & 1.100 & 4.100 & 55.000 \\
\hline $\begin{array}{l}\text { Scholle et al.- } \\
1\end{array}$ & 2011 & 22 & 2.800 & 1.289 & 0.000 & 0.001 & NA & NA & 16.000 & 55.000 \\
\hline $\begin{array}{l}\text { Scholle et al.- } \\
2\end{array}$ & 2011 & 23 & 1.500 & 2.421 & 0.000 & 0.001 & NA & NA & 36.000 & 43.500 \\
\hline $\begin{array}{l}\text { Scholle et al.- } \\
3\end{array}$ & 2011 & 25 & 1.100 & 1.055 & 0.000 & 0.001 & NA & NA & 60.000 & 48.000 \\
\hline $\begin{array}{l}\text { Scholle et al.- } \\
4\end{array}$ & 2011 & 34 & 0.900 & 0.938 & 0.000 & 0.001 & NA & NA & 96.000 & 50.000 \\
\hline $\begin{array}{l}\text { Scholle et al.- } \\
5\end{array}$ & 2011 & 33 & 0.400 & 0.469 & 0.000 & 0.001 & NA & NA & 138.000 & 39.400 \\
\hline $\begin{array}{l}\text { Scholle et al.- } \\
6\end{array}$ & 2011 & 23 & 0.500 & 0.664 & 0.000 & 0.001 & NA & NA & 150.000 & 30.400 \\
\hline $\begin{array}{l}\text { Scholle et al.- } \\
7\end{array}$ & 2011 & 24 & 0.100 & 0.313 & 0.000 & 0.001 & NA & NA & 182.400 & 50.000 \\
\hline $\begin{array}{l}\text { Scholle et al.- } \\
8\end{array}$ & 2011 & 25 & 0.100 & 0.508 & 0.000 & 0.001 & NA & NA & 202.800 & 56.000 \\
\hline Traeger et al. & 2005 & 66 & & & 0.010 & 0.030 & NA & NA & 79.200 & 64.000 \\
\hline & 2004 & 70 & & & & 0.025 & NA & NA & 94.800 & 43.000 \\
\hline Verhulst et al. & 2007 & 60 & 0.850 & 1.060 & 0.060 & 0.160 & NA & NA & 140.400 & 47.000 \\
\hline Wang et al.-1 & 2016 & 33 & 0.700 & 0.820 & 0.080 & 0.120 & 0.020 & 0.060 & 54.000 & 54.600 \\
\hline Wang et al.-2 & 2016 & 66 & 0.280 & 0.420 & 0.070 & 0.140 & 0.030 & 0.110 & 109.200 & 57.600 \\
\hline Wong et al. & 2004 & 11 & 0.100 & 0.100 & 0.000 & 0.001 & & NA & 67.200 & 63.600 \\
\hline
\end{tabular}

Fig. 1 | KV 14 


\begin{tabular}{|c|c|c|c|c|c|c|c|c|c|c|}
\hline \multirow[t]{3}{*}{ Study } & \multirow[t]{3}{*}{ Year } & \multirow{3}{*}{$\begin{array}{l}\text { Group } \\
\mathrm{N}\end{array}$} & \multicolumn{6}{|c|}{ Mean apnea duration during sleep, sec } & \multirow{3}{*}{$\begin{array}{l}\text { Mean } \\
\text { age, } \\
\text { mo }\end{array}$} & \multirow{3}{*}{$\begin{array}{l}\text { Percent } \\
\text { boys }\end{array}$} \\
\hline & & & \multicolumn{2}{|c|}{$\begin{array}{l}\text { Central } \\
\text { apneas }\end{array}$} & \multicolumn{2}{|c|}{\begin{tabular}{|l|}
$\begin{array}{l}\text { Obstructive } \\
\text { apneas }\end{array}$ \\
\end{tabular}} & \multicolumn{2}{|c|}{$\begin{array}{l}\text { Mixed } \\
\text { apneas }\end{array}$} & & \\
\hline & & & Mean & SD & Mean & SD & Mean & SD & & \\
\hline $\begin{array}{l}\text { Brockmann et } \\
\text { al.-1 }\end{array}$ & 2013 & 37 & 5.200 & 4.200 & 5.200 & 2.975 & 6.900 & 3.575 & 1.000 & 59.000 \\
\hline $\begin{array}{l}\text { Brockmann et } \\
\text { al.-2 }\end{array}$ & 2013 & 37 & 5.100 & 1.600 & 5.100 & 1.400 & 6.800 & 2.825 & 3.000 & 59.000 \\
\hline Coh et al. & 2000 & 10 & NA & NA & NA & NA & NA & NA & 72.000 & 30.000 \\
\hline Daftary et al. & 2019 & 30 & 5.700 & 0.950 & 5.600 & 0.950 & 6.600 & 1.225 & 0.630 & 40.000 \\
\hline Kato et al.-1 & 2000 & 63 & NA & NA & 5.500 & 2.500 & 8.000 & 2.875 & 1.500 & 35.000 \\
\hline Kato et al.-2 & 2000 & 521 & NA & NA & 5.000 & 1.500 & 7.000 & 1.750 & 2.500 & 47.000 \\
\hline Kato et al.-3 & 2000 & 238 & NA & NA & 5.000 & 1.417 & 6.300 & 1.500 & 3.500 & 42.000 \\
\hline Kato et al.-4 & 2000 & 137 & NA & NA & 5.000 & 1.750 & 7.000 & 1.167 & 4.500 & 49.600 \\
\hline Kato et al.-5 & 2000 & 64 & NA & NA & 4.500 & 2.375 & 6.000 & 1.250 & 5.500 & 50.000 \\
\hline $\begin{array}{l}\text { Montgomery- } \\
\text { Downs et al.- } \\
1\end{array}$ & 2013 & 153 & NA & NA & NA & $\mathrm{NA}$ & NA & NA & 58.800 & 54.900 \\
\hline $\begin{array}{l}\text { Montgomery- } \\
\text { Downs et al.- } \\
2\end{array}$ & 2013 & 388 & NA & NA & NA & NA & NA & NA & 81.600 & 51.500 \\
\hline Moss et al. & 2005 & 50 & NA & NA & NA & NA & 13.100 & 3.600 & 121.200 & 52.000 \\
\hline Sanchez et al. & 2006 & 320 & 27.600 & 6.400 & NA & NA & NA & NA & 4.100 & 55.000 \\
\hline $\begin{array}{l}\text { Scholle et al.- } \\
1\end{array}$ & 2011 & 22 & NA & NA & NA & $\mathrm{NA}$ & NA & NA & 16.000 & 55.000 \\
\hline $\begin{array}{l}\text { Scholle et al.- } \\
2\end{array}$ & 2011 & 23 & NA & NA & NA & NA & NA & NA & 36.000 & 43.500 \\
\hline $\begin{array}{l}\text { Scholle et al.- } \\
3\end{array}$ & 2011 & 25 & NA & NA & NA & $\mathrm{NA}$ & NA & NA & 60.000 & 48.000 \\
\hline $\begin{array}{l}\text { Scholle et al.- } \\
4\end{array}$ & 2011 & 34 & NA & NA & NA & NA & NA & NA & 96.000 & 50.000 \\
\hline $\begin{array}{l}\text { Scholle et al.- } \\
5\end{array}$ & 2011 & 33 & NA & NA & NA & NA & NA & NA & 138.000 & 39.400 \\
\hline $\begin{array}{l}\text { Scholle et al.- } \\
6\end{array}$ & 2011 & 23 & NA & NA & NA & NA & NA & NA & 150.000 & 30.400 \\
\hline $\begin{array}{l}\text { Scholle et al.- } \\
7\end{array}$ & 2011 & 24 & NA & NA & NA & NA & NA & NA & 182.400 & 50.000 \\
\hline $\begin{array}{l}\text { Scholle et al.- } \\
8\end{array}$ & 2011 & 25 & NA & NA & NA & NA & NA & NA & 202.800 & 56.000 \\
\hline Traeger et al. & 2005 & 66 & NA & NA & NA & NA & NA & NA & 79.200 & 64.000 \\
\hline Uliel et al. & 2004 & 70 & 11.800 & 3.000 & 10.300 & 2.100 & NA & NA & 94.800 & 43.000 \\
\hline Verhulst et al. & 2007 & 60 & NA & NA & 6.300 & 2.300 & NA & NA & 140.400 & 47.000 \\
\hline Wang et al.- -1 & 2016 & 33 & 11.890 & 6.360 & 4.710 & 6.330 & 2.090 & 5.720 & 54.000 & 54.600 \\
\hline Wang et al.-2 & 2016 & 66 & 9.630 & 7.830 & 6.080 & 9.560 & 2.790 & 7.460 & 109.200 & 57.600 \\
\hline Wong et al. & 2004 & 11 & NA & NA & NA & NA & NA & NA & 67.200 & 63.600 \\
\hline
\end{tabular}

\section{Fig. 2 | KV 14 ॥}

Methods: A systematic literature search in MEDLINE, Web of Science and Google Scholar was performed in February 2020 to identify studies conducted in 2000 through 2020 that described normative values of respiratory events during sleep in infants and children based on polysomnographic investigations. Using the PRISMA reporting guidelines, thirteen studies covering polysomnographic findings of 2563 participants reporting the values of the frequencies and durations of central, obstructive and mixed apneas during sleep in normal infants and children were identified. The mean age of the participants in the studies ranged from 0.6 through 140.4 months. Proportions of boys in the studies ranged between 30 and $64 \%$. A meta-analysis was performed for each of the measurements using OpenMetaAnalyst software (Brown University School of Public Health, Providence RI, US) in random-effect models. Reported or calculated mean values and standard deviations were analysed.

Results: - Fig. 1 summarizes the findings on the mean frequencies of the different types of apneas per $1 \mathrm{~h}$ of sleep time (apnea indices) across the studies. Meta-regression found statistically significant association between mean central apnea index and mean child age $(b=-0.012 ; p<0.001)$, mean obstructive apnea index and mean child age $(b=-0.002 ; p<0.001)$, mean mixed apnea index and mean child age $(b=-0.002 ; p=0.019)$. No statistically significant associations were found between the mean values of the apnea indices and the percent of boys across the studies. 0 Fig. 2 is the summary of the mean apneas duration. Meta-regression failed to find associations between the mean central apnea length $(p=0.982)$, the mean mixed apnea length $(p=0.646)$ and the mean age of children. Significant association was found between the mean obstructive apnea length and the mean age of children $(b=0.019 ; p=0.01)$. No significant associations were found with percent of boys.

Conclusions: The frequencies of apneas diminish with advancing age and do not depend on sex. Apneas lengths are stable across age group, except obstructive apneas that may become longer in older children. Apneas lengths do not depend on sex.

Keywords: Apnea, Children, Infants, Meta-analysis, Reference values

\section{KV 15}

\section{Schlafbezogene Atmungsstörungen bei idiopathischer Lungenfibrose}

*S. Herkenrath, L. Hagmeyer, M. Treml, N. Anduleit, W. J. Randerath

Institut für Pneumologie an der Universität zu Köln/Krankenhaus Bethanien, Solingen, Deutschland

Fragestellung: Schlafbezogene Atmungsstörungen (SBAS) werden bei der idiopathischen Lungenfibrose (IPF) noch wenig beachtet und ihre Bedeutung ist für das Outcome ist unklar. Wir führten diese Studie durch, um die Prävalenz von SBAS und die assoziierte Symptomatik sowie Auswirkungen von SBAS bei IPF auf das Schlafprofil, die hypoxische Last und kardiopulmonale Leistungsfähigkeit zu untersuchen.

Patienten und Methoden: In dieser diagnostischen Querschnittsstudie wurden unselektierte IPF-Patienten ohne Langzeitsauerstofftherapie in stabilem Zustand rekrutiert und einer Polysomnographie zugeführt. Die Symptomatik wurde mittels „Epworth Sleepiness Scale“ (ESS) und „Pittsburgh Sleep Quality Index“ (PSQI) erfasst. Alle Patienten unterzogen sich zudem einer Spiroergometrie, Bodyplethysmographie sowie CO-Diffusionsmessung (single breath). SBAS wurden definiert als ein Apnoe-Hypopnoe-Index (AHI) $\geq 15 / \mathrm{h}$ oder $\geq 5 / \mathrm{h}$ beim Vorliegen einer ESS-Punktzahl von $>9$.

Ergebnisse: Von 45 eingeschlossenen IPF-Patienten wurden bei 22 SBAS diagnostiziert (davon 81 \% OSA). Anthropometrische Daten und Lungenfunktion waren zwischen Patienten mit und ohne SBAS sehr vergleichbar, ebenso ESS und PSQI-Score (• Tab. 1). Patienten mit SBAS zeigten einen geringeren Tiefschlafanteil (• Abb. 1a) und eine höhere hypoxische Last (- Abb. 1b) sowie gemäß Spiroergometrie eine geringere Leistungsfähigkeit nach Watt (• Abb. 2a), eine geringere Sauerstoffaufnahme an der an-

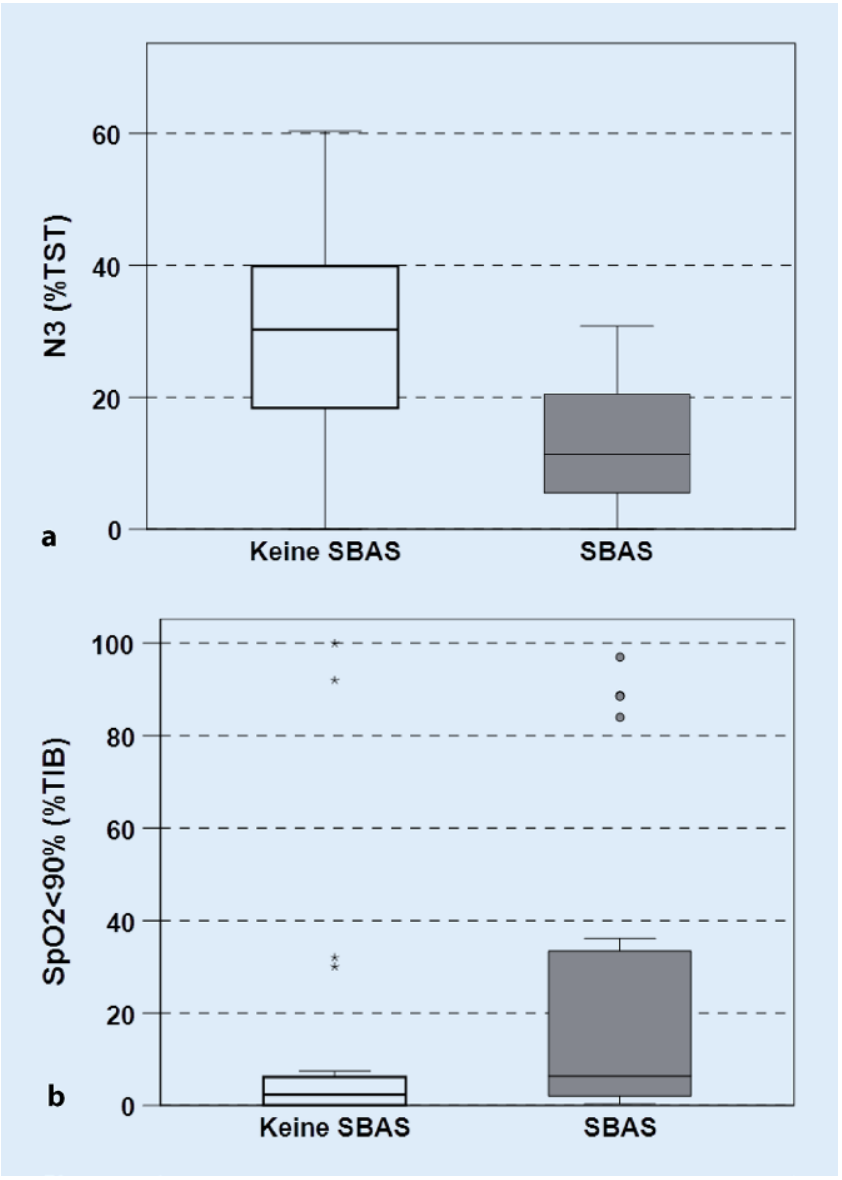

Abb. 1 | KV 15 A 

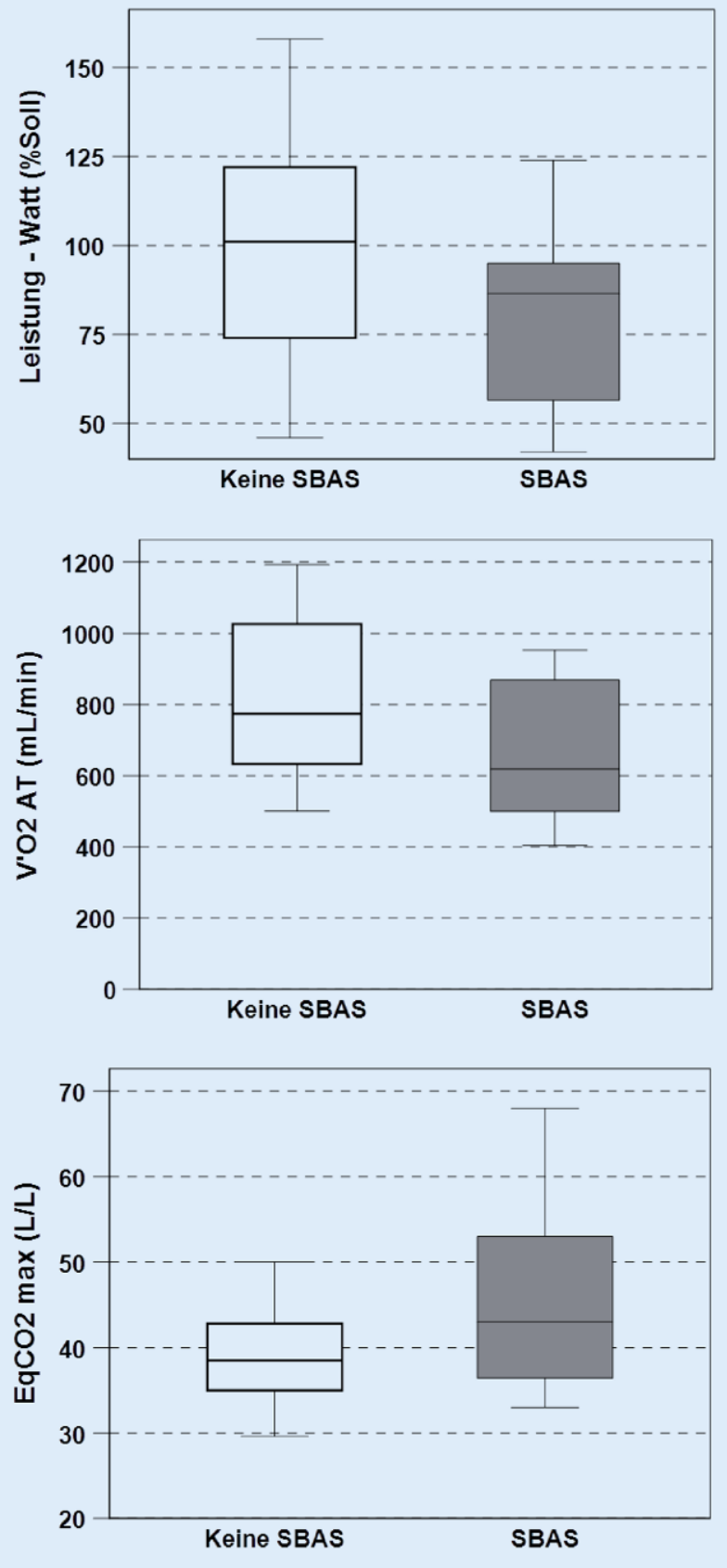

Abb. 2 | KV 15 A

aeroben Schwelle ( $\bullet$ Abb. 2 b) sowie ein höheres CO2-Atemäquivalent (- Abb. 2c).

Schlussfolgerungen: SBAS bei IPF-Patienten sind von hoher Prävalenz. IPF mit SBAS weisen eine kompromittierte globale kardiopulmonale Leistungsfähigkeit und zeigen basierend auf dem erhöhten Atemäquivalent für CO2 Hinweise auf eine pulmonale Hypertonie im Vergleich zur IPF ohne SBAS. Die größere hypoxische Last lässt außerdem ein erhöhtes Mortalitätsrisiko vermuten. Eine SBAS-typische Symptomatik liegt häufig nicht vor, weshalb mindestens eine 2-Kanaldiagnostik im Rahmen von Screeningmaßnahmen erforderlich ist.

Schlüsselwörter: Idiopathische Lungenfibrose, Schlafbezogene Atmungsstöru, Obstruktive Schlafapnoe, Schläfrigkeit, Spiroergometrie

\begin{tabular}{|c|c|c|c|}
\hline & Keine SBAS & SBAS & $\mathrm{p}$ \\
\hline $\begin{array}{l}\text { Geschlecht } \\
(\mathrm{m} / \mathrm{w})\end{array}$ & $6 / 17$ & $5 / 17$ & - \\
\hline Alter (Jahre) & 74 & 77 & 0,75 \\
\hline $\mathrm{BMI}\left(\mathrm{kg} / \mathrm{m}^{2}\right)$ & 27,5 & 26,4 & 0,45 \\
\hline FEV1 (\%Soll) & 78 & 78 & 0,80 \\
\hline FVC (\%Soll) & 74 & 68 & 0,98 \\
\hline ESS & 4 & 5 & 0,73 \\
\hline PSQI & 6 & 8 & 0,65 \\
\hline N3 (\%TST) & 30 & 11 & 0,00 \\
\hline $\begin{array}{l}\mathrm{tSpO2<90 \%} \\
\text { (\%TIB) }\end{array}$ & 2,3 & 6,3 & 0,04 \\
\hline $\begin{array}{l}\text { Max. Watt-Leis- } \\
\text { tung (\%Soll) }\end{array}$ & 101 & 87 & 0,04 \\
\hline $\begin{array}{l}\text { V'O2 an AT (mL/ } \\
\text { min) }\end{array}$ & 774 & 619 & 0,04 \\
\hline EqCO2 (L/L) & 39 & 43 & 0,04 \\
\hline
\end{tabular}

\section{KV 16}

\section{Kontaktloses System zur Erkennung von Apnoe}

${ }^{*}$ M. Gaiduk ${ }^{1,2}$, R. Seepold ${ }^{1,3}$, T. Penzel $^{4}$

${ }^{1}$ HTWG Konstanz, Konstanz, Deutschland; ' 2 University of Seville, Sevilla, Spanien; ${ }^{3}$. M. Sechenov First Moscow State Medical University, Moskau, Russische Föderation; ${ }^{4}$ Charité - Universitätsmedizin Berlin, Berlin, Deutschland

Fragestellung: Die Schlafapnoe ist eine häufig auftretende Schlafstörung, die unterschiedliche Auswirkungen auf unseren Alltag hat; so wurde z. B. über eine Tagesschläfrigkeit von etwa $25 \%$ der Patienten mit obstruktiver Schlafapnoe (OSA) berichtet [1]. Ziel dieser Arbeit ist die Entwicklung eines Systems, das eine nichtinvasive Erkennung der Schlafapnoe in häuslicher Umgebung ermöglichen soll.

Patienten und Methoden: Die Studie wurde mit 20 Patienten (10 männliche und 10 weibliche) durchgeführt. Die Patienten hatten ein Körpergewicht im Bereich von 45-90 kg und eine Körpergröße zwischen $156-183 \mathrm{~cm}$. Das entwickelte System besitzt eine Hardware- und eine Software-Komponente, die Apnoe erkennen und visualisieren kann. Die Hardware besteht aus Drucksensoren, die über Analog/Digital-Wandler, Verstärker- und Filterschaltkreise an eine Recheneinheit angeschlossen sind. Diese überträgt die vorverarbeiteten Messdaten an eine Software, die die weitere Auswertung der Messdaten übernimmt. Die Ergebnisse werden anschließend über ein Display angezeigt.

Ergebnisse: Insgesamt wurden 100 Apnoe-Ereignisse mit einer Länge von je 30 s simuliert. Die erreichte Genauigkeit von Apnoe-Ereignissen liegt bei $91 \%$, wobei die durchschnittlich gemessene Erkennungsverzögerung bei nur etwa $15 \mathrm{~s}$ liegt. Dies ist mit dem Stand der Technik vergleichbar [2] aber das vorgestellte System basiert auf einem neuen Ansatz und hat mehrere bedeutende Vorteile, wie zum Beispiel die Möglichkeit zur langfristig kontaktlosen Anwendung in häuslichem Umfeld bei niedrigen Materialkosten (ca. $110 €$ ). Es bietet auch eine Erweiterbarkeit und Anschlussfähigkeit für externe Geräte an.

Schlussfolgerungen: Die erzielten Ergebnisse bestätigen ein Potenzial für eine zukünftige Nutzung. Das System ist unauffällig zu platzieren und es kann als eigenständiges Gerät eingesetzt werden; die Verbindung zu externen Geräten ist möglich und wird drahtlos implementiert. Neben der Apnoe-Erkennung könnte dieses System auch für die Überwachung der Atmung benutzt werden. Dazu würden zusätzliche Filterverfahren, wie 
z. B. die kontinuierliche Wavelet-Transformation, benötigt. Darüber hinaus könnte der vorgeschlagene Ansatz als Teil eines umfangreicheren Systems z. B. für eine Schlafstudie verwendet werden, indem man den Ansatz in [3] mit diesem System erweitert.

Schlüsselwörter: Apnoe, Kontaktloses Hardware-System, Überwachung der Atmung, Schlafstudie, Nichtinvasives System

\section{Literatur}

1. Tkacova R, Dorkova Z (2010) Clinical presentations of OSA in adults. Eur Respir Monogr 50:86-103

2. Mendonça F, Mostafa SS, Ravelo-García AG et al (2019) A Review of Obstructive Sleep Apnea Detection Approaches. leee J Biom Health Inf 23(2):825-837

3. Gaiduk M, Penzel T, Ortega JA, Seepold R (2018) Automatic sleep stages classification using respiratory, heart rate and movement signals. Phys Meas 39:

\section{KV 17}

\section{Virtual face-to-face triage visits to waitlisted patients-learning lessons from the COVID-19 shutdown}

${ }^{*}$ S. McWilliams ${ }^{1}$, R. Friedlander ${ }^{2,3}$, D. Wensley ${ }^{4,5},{ }^{*}$ O. Ipsiroglu ${ }^{1,6}$

${ }^{1} \mathrm{H}$-Behaviours Research Lab (Previously Sleep/Wake-Behaviours Research Lab), BC Children's Hospital Research Institute, Pediatrics, Vancouver, Canada; ${ }^{2}$ Neuropsychiatry Clinic, BC Children's Hospital, Psychiatry, Vancouver, Canada; ${ }^{3}$ Self Injurious Behaviours Clinic, BC Children's Hospital, Psychiatry, Vancouver, Canada; ${ }^{4}$ Sleep Program, BC Children's Hospital, Pediatrics, Vancouver, Canada; ${ }^{5}$ Respiratory Medicine, BC Children's Hospital, Pediatrics, Vancouver, Canada; ${ }^{6}$ Sleep Program, Sleep/Wake Behaviour Clinic, BC Children's Hospital, Pediatrics, Vancouver, Canada

Objectives: The COVID-19 pandemic is magnifying existing gaps in service delivery and increasing wait lists. The mandate of the Sleep/WakeBehaviour Clinic in Child \& Adolescent Psychiatry is to support referring sub-specialty services and community providers primarily through $\mathrm{s} / \mathrm{w}$ assessments from pediatric mental health perspectives. We analyzed the workload and anticipated wait time for clinical assessment and are suggesting a new triage system for coping with increased patient numbers and waitlist times.

Methods: Prior to the shutdown, we documented the amount of time required for new and person/phone follow-up assessments of children with neurodevelopmental and -psychiatric disorders (incl. team meetings; chart/lab results reviews; dictations) in a $20 \mathrm{hrs} / \mathrm{wk}$ ambulatory service, and reviewed expected challenges in clinical management of the $185 \mathrm{pa}-$ tients currently waitlisted.

Results: 81 (age range: 2-19) patients were scheduled over 5 weeks requiring on average $2.4 \mathrm{~h} /$ patient $(24$ days/40 h), 20/81 patients were "red flagged" (definition: 911-call eligible at any worsening). The current wait list includes 185 new patients, predicting in the best case a 18-24 week wait time, if the clinic runs with $100 \%$ care time commitment every week. Conclusions: The expected 18-24 week wait time calculated from this " 81 patients over 5 weeks" analysis is likely to be much longer, as absences were not taken into account. In a real life scenario, the waitlist would likely be around 8-14 months, which, from a patient perspective, is unacceptable. We suggest a novel triage concept consisting of a structured phonebased pre-screening (by booking clerk) and a virtual face-to-face triage visit to patients (by physician/nurse), including triage support to community health care providers via a written individualized report. Guided by a needs assessment concept, patients are coded red, yellow, and green, the latter standing for problems which could potentially be solved in the community by clinicians with guidance (e.g. providing evidence based interventions). The implementation of first line measures for category "green" (e.g. screening for familial iron deficiency) and harmonizing surveillance measures will enhance knowledge dissemination.

Keywords: Clinical sleep assessments, Wait list, Insomnia, Pediatrics, Child \& Adolescent Psychiatry

\section{KV 18}

Auswirkung der kognitiven Verhaltenstherapie auf die Ausprägung komorbider Insomnien

\section{${ }^{*}$ R. Witt ${ }^{1}$, H. G. Weeß ${ }^{2}$, T. In-Albon ${ }^{3}$}

'Universität Kassel, Klinische Psychologie und Psychotherapie, Kassel, Deutschland; ${ }^{2}$ Pfalzklinikum, Interdisziplinäres Schlafzentrum, Klingenmünster, Deutschland; ${ }^{3}$ Universität Landau, Klinische Psychologie und Psychotherapie des Kindes- und Jugendalters, Landau, Deutschland

Fragestellung: Die Wirksamkeit der kognitiven Verhaltenstherapie für Insomnie (KVT-I) gilt als bestätigt. Viele wiss. Fachverbände betrachten sie als first-line Therapie. Ihr positiver Einfluss ist auch für komorbide Insomnien belegt. Einige Studien gehen von einem moderierenden Einfluss der Emotionsregulation aus. Diese Studie widmet sich der Evaluation einer

Table 1 | KV 17

\begin{tabular}{|c|c|c|c|c|c|c|c|}
\hline & \multirow[t]{2}{*}{ New patients } & \multicolumn{2}{|l|}{ Follow ups } & \multirow[t]{2}{*}{ Transition } & \multirow[t]{2}{*}{ No show } & \multirow{2}{*}{$\begin{array}{l}\text { Total \# of schedulded } \\
\text { assessments/week }\end{array}$} & \multirow{2}{*}{$\begin{array}{l}\text { Meetings (Sleep/Neuro- } \\
\text { psychiatric Pharmacist) } \\
\text { Total \# meetings/week }\end{array}$} \\
\hline & & 1 hour & $1 / 2$ hour & & & & \\
\hline Week 1 & 1 & 10 & 3 & 1 & 1 & 16 (7 red flags) & 2 \\
\hline Week 2 & 1 & 11 & 7 & & & 19 (3 red flags) & 3 \\
\hline Week 3 & 5 & 3 & 1 & 1 & & 10 (2 red flags) & 2 \\
\hline Week 4 & 8 & 5 & 1 & & & 14 (5 red flags) & 2 \\
\hline Week 5 & 5 & 6 & 10 & & 1 & 22 (3 red flags) & 4 \\
\hline Total \# assessments & 20 (30 hrs) & 35 (35 hrs) & 22 (11 hrs) & 2 (2 hrs) & 2 (2 hrs) & $\begin{array}{l}81 \text { ( } 20 \text { red flags) } \\
\text { assessments ( } 80 \mathrm{hrs})\end{array}$ & 13 meetings ( $24 \mathrm{hrs}$ ) \\
\hline \multicolumn{8}{|c|}{ Additional hours outside of the assessment time } \\
\hline $\begin{array}{l}\text { Chart review/lab } \\
\text { results (hrs) }\end{array}$ & 20 & 17.5 & 2.75 & 0.75 & 1 & & \\
\hline $\begin{array}{l}\text { Dictation/Assess- } \\
\text { ment review (hrs) }\end{array}$ & 20 & 17.5 & 2.75 & 0.75 & 0.5 & & \\
\hline $\begin{array}{l}\text { Total time (hrs) for } \\
\text { assessment + review }\end{array}$ & 70 & 70 & 16.5 & 3.5 & 3.5 & $\begin{array}{l}163.5 \mathrm{hrs} / 81 \text { patients } \\
\text { over } 5 \text { weeks }\end{array}$ & $\begin{array}{l}30 \mathrm{hrs} / 81 \text { patients over } 5 \\
\text { weeks }\end{array}$ \\
\hline
\end{tabular}

Total hours spent for 81 schedulded appointments over a 5 week period, including assessment/meeting/chart review/dictation = 193.5 hours 
KVT-I-Kurzzeitintervention, wie sie am Pfalzklinikum angeboten wird. Neben der Veränderung der Insomnieschwere wird in dieser Studie der Einfluss der KVT-I auf begleitende Depressions- und Angstsymptome untersucht, sowie die Emotionsregulation als moderierender Faktor berücksichtigt. Es wird angenommen, dass die KVT-I nicht nur die Insomnie, sondern auch Depressivität und Angst signifikant reduzieren kann. Die Hypothese ist, dass adaptive Emotionsregulationsstrategien zu einer stärkeren Reduktion der Insomnie mit komorbiden Erkrankungen führen, als maladaptive Emotionsregulationsstrategien.

Patienten und Methoden: Insgesamt 40 Teilnehmer (21w, Alter 54,2 $\pm 12,6$ Jahre) wurden in drei KVT-I Maßnahmen akquiriert und erhielten in einem Prä-Post Design den Insomnie Severity Index (ISI), den Emotion Regulation Questionnaire (ERQ), sowie das Beck-Depressions Inventar (BDI) und das State-Trait-Anxiety Inventory (STAI). Der Einfluss der Emotionsregulation wurde mithilfe eines korrelativen Ansatzes untersucht.

Ergebnisse: Im Prä-Post Vergleich konnte die Insomnieausprägung $(d=0,99)$ und Depressivität $(d=0,63)$ signifikant reduziert werden, während sich bei der Trait- und State Angst keine signifikanten Veränderungen zeigten. Die Emotionsregulation stellt in dieser Studie keine signifikante Einflussgröße auf die Reduktion von Insomnie, Depressivität und Angst dar. Trotz fehlender statistischer Signifikanz lässt sich ein Unterschied zwischen der adaptiven und maladaptiven Emotionsregulation erkennen. Hierbei ging eine erhöhte adaptive Emotionsregulation tendenziell mit geringeren Prä-Post Differenzen einher.

Schlussfolgerungen: Die Befunde sprechen für eine positive Beeinflussung der Insomnie mit komorbiden Erkrankungen durch die KVT-I. Dieser Effekt scheint unabhängig von der Ausprägung der Emotionsregulation aufzutreten. In zukünftigen Studien sollten größere Stichproben mithilfe von regressionsanalytischen Ansätzen in einem Längsschnittdesign untersucht werden. Weiterführend empfiehlt es sich den Einfluss der adaptiven Emotionsregulation durch einen experimentellen Ansatz von der maladaptiven Emotionsregulation abzugrenzen und differenziert zu betrachten. Schlüsselwörter: KVT-I, Insomnie, Depression, Angst, Emotionsregulation

\section{KV 19 \\ „Gesunder Schlaf durch innere Ordnung" - Entwicklung, Implementierung und Evaluierung eines 3-wöchigen Programms zur Sekundärprävention bei nicht organisch bedingten Schlafstörungen}

M. Stier-Jarmer, ${ }^{*} D$. Frisch

Ludwig-Maximilians-Universität München, Lehrstuhl für Public Health und Versorgungsforschung, Fachbereich Kurortmedizin und Prävention, München, Deutschland

Fragestellung: Ein- und Durchschlafstörungen, die mit Tagesmüdigkeit sowie sozialen und beruflichen Beeinträchtigungen einhergehen, zählen heute zu den häufig vorkommenden Beschwerden in der Bevölkerung der modernen Industriestaaten. Schlafstörungen sind mit körperlichen und psychischen Gesundheitsstörungen und daraus resultierenden volkswirtschaftlichen Kosten verbunden. Ziel dieser Studie war es, ein Präventionsprogramm zum Thema „nicht organisch bedingte Schlafstörungen“ $\mathrm{zu}$ entwickeln, bei betroffenen Personen anzuwenden und zu evaluieren. Patienten und Methoden: Das Programm wurde auf Basis einer ambulanten Kneippkur mit den Schwerpunkten Ordnungstherapie, Hydrotherapie und Bewegungstherapie erstellt. Es verknüpft Psychoedukation und Entspannungsverfahren mit Elementen der Hydrotherapie und aktivitätsfördernden Bewegungskomponenten. Ziel ist es, seit längerem anhaltende, nicht organisch bedingte Ein- und/oder Durchschlafstörungen zu reduzieren bzw. langfristig zu beheben.

Die Evaluierung des Programms erfolgte im Rahmen einer randomisierten kontrollierten Studie mit Wartegruppendesign und 6-monatiger Nachbeobachtung. Primäre Zielgröße ist die Schlafqualität (PSQI) nach 6 Monaten. Sekundäre Zielgrößen sind u.a. Wohlbefinden (WHO-5), Subjektiver Gesundheitszustand (EQ-5D-5L), Chronischer Stress (TICS),
Dysfunktionale Überzeugungen und Einstellungen zum Schlaf (DBAS16), psychische Symptome (ISR) und Gesundheitsverhalten.

Ergebnisse: Die 100 Studienteilnehmer (Interventionsgruppe - IG =49; Wartekontrollgruppe - WG $=51$ ) waren im Mittel 56 Jahre alt und meist weiblich (84\%). Der Anteil der schlechten Schläfer (PSQI 6-10) und der Personen mit chronischer Schlafstörung (PSQI > 10) betrug zu Beginn $98 \%$ in beiden Studiengruppen. Nach 6 Monaten war deren Anteil auf 71,7\% (IG) bzw. 85,7\% (WG) gesunken. Insbesondere die chronische Schlafstörung war in der IG nur noch bei $28,3 \%$ (WG 40,8\%) der Teilnehmer zu finden. $83 \%$ der IG und 67,3\% der WG Teilnehmer zeigten nach 6 Monaten verbesserte PSQI-Werte (IG - 3,53; WG - 1,28). In der WG wurde allerdings zu keinem Zeitpunkt eine klinisch relevante Verbesserung (mind. 3 Punkte) erreicht. Insgesamt zeigten sich in der IG im Vergleich zur WG signifikante Verbesserungen bei Schlafqualität, Wohlbefinden und chronischem Stress, die über einen Zeitraum von bis zu 6 Monaten anhielten.

Schlussfolgerungen: Zusammengefasst zeigt das 3-wöchige Programm "Gesunder Schlaf durch innere Ordnung" in Füssen sichtlich positive Effekte bei Schlafqualität, Wohlbefinden, chronischer Stressbelastung und weiteren Zielgrößen und sollte für Menschen mit nicht organisch bedingten Schlafstörungen weiter angeboten werden. Zur nachhaltigen Stabilisierung des Behandlungserfolgs und zur langfristigen Aufrechterhaltung der erreichten Verhaltens- und Lebensstiländerungen könnte in Zukunft die Durchführung und Evaluation von Auffrischungsprogrammen in Erwägung gezogen werden. Zukünftige Studien sollten zudem die Langzeiteffekte des Programms untersuchen.

Schlüsselwörter: Insomie, Kneippkur, Hydrotherapie, Lebensstil, Kurort

\section{KV 20}

Die obere Atemwegsstimulation bei obstruktiver Schlafapnoe - Ein interkontinentaler Vergleich der Ergebnisse unter Berücksichtigung des BMls

\section{*A. Steffen', B. Hofauer', J. T. Maurer', J. U. Sommer', T. Verse ${ }^{4}$, C. Heiser ${ }^{5}$}

'Universität zu Lübeck, Klinik für HNO-Heilkunde, Lübeck, Deutschland; 2Universität Freiburg, Klinik für HNO-Heilkunde, Freiburg, Deutschland; ${ }^{3}$ Universitätsmedizin Mannheim, Klinik für HNO-Heilkunde, Mannheim, Deutschland; ${ }^{4}$ Helios-Universitätsklinikum Wuppertal, Universität Witten/ Herdecke, Klinik für HNO-Heilkunde, Wuppertal, Deutschland; ${ }^{5}$ Klinikum rechts der Isar, Technische Universität München, Klinik und Poliklinik für Hals-Nasen-Ohrenheilkunde, München, Deutschland

Fragestellung: Die selektive obere Atemwegsstimulation (UAS) ist eine etablierte Therapieoption bei obstruktiver Schlafapnoe (OSA) und PAPIntoleranz in Europa und den USA. Das internationale ADHERE-Register dokumentiert die 1-Jahresnachsorge (M12). Unser Ziel ist es, die Ergebnisse der UAS zwischen US-amerikanischen und europäischen Versorgungsstrukturen zu vergleichen und insbesondere das Übergewicht zu berücksichtigen.

Patienten und Methoden: Die Einschlusskriterien waren ein AHI $\geq 15 / \mathrm{h} \leq 65 / \mathrm{h}$ mit vorliegenden Ergebnissen zur M12. Die Daten wurden nach Kontinenten (EU vs. USA) sowie bzgl. des Übergewichts (BMI $\leq 32$ vs $32<\mathrm{BMI} \leq 35 \mathrm{~kg} / \mathrm{m}^{2}$ ) gruppiert. Als Äquivalenzgrenzen wurde für den AHI -5 bis 5 sowie für den ESS -2 und 2 festgelegt.

Ergebnisse: Etwa 1900 Patienten sind in das Register eingeschlossen (5/2020) und 840 absolvierten die M12. In den EU-Zentren (Deutschland, Belgien, Niederlande) wurden $38 \%$ und in den USA $62 \%$ rekrutiert. Beide Gruppen ähneln sich im initialen AHI und M12-AHI (EU

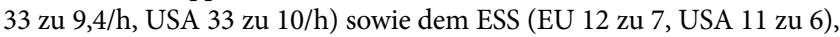
aber auch in den Sher-Kriterien (EU $72 \%$, USA $68 \%$ ). Die durchschnittliche AHI und ESS-Differenz verblieb innerhalb der Äquivalenzgrenzen (AHI: $0,3, \mathrm{CI}[-1,10,1,70]$, ESS $\left.6,8 \mathrm{e}^{\wedge}-5, \mathrm{CI}[-1,08,-1,00]\right)$. Überwiegend war der BMI $\leq 32 \mathrm{~kg} / \mathrm{m}^{2}$; bei $15 \%$ lag er zwischen 32 und $35 \mathrm{~kg} / \mathrm{m}^{2}$; EU zu USA kein Unterschied. Die AHI-Reduktion unterschied sich nicht, aber das Therapieansprechen nach Sher (72 zu $60 \%$; $p=0,02)$. Der ESS zu M12 unterschied sich nicht. 
Schlussfolgerungen: Die Ergebnisse des ADHERE-Registers zeigen eine hohe Therapieeffektivität der UAS sowohl in europäischen als auch USamerikanischen Gesundheitsstrukturen. Während sich das Therapieansprechen nach Sher in den BMI-Gruppen unterscheidet, sind die AHIReduktionen und die finalen Tagesschläfrigkeitswerte gleich.

Schlüsselwörter: Schlafapnoe, CPAP-Intoleranz, Hypoglossus-Stimulation, Zungenschrittmacher, Schlafchirurgie

\section{KV 21}

Pitolisant evaluation in obstructive sleep apnea patients treated by nasal continuous positive airway pressure but still complaining of excessive daytime sleepiness

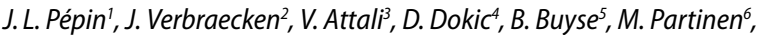
T. Saaresranta ${ }^{7}$, I. Fietze ${ }^{8}$, O. Georgiev ${ }^{9}$, G. Belev ${ }^{10}$, R. Tamisier ${ }^{11}$, I. Lecomte ${ }^{12}$, J. M. Lecomte ${ }^{12}$, P. Lévy ${ }^{11}$, J. C. Schwartz ${ }^{13}$, Y. Dauvilliers ${ }^{14},{ }^{*}$ C. Caussé12 'Grenoble University Hospital, ADTSAS, Grenoble, France; ${ }^{2}$ Universtiair Multidisciplinair Slaap Centrum, Slaap Centrum, Edegem-Antwerp, Belgium; ${ }^{3}$ Sorbonne Université, INSERM, UMRS1158 Neurophysiologie Respiratoire Expérimentale et Clinique, Paris; AP-HP, Groupe Hospitalier Universitaire APHP-Sorbonne Université, Pitié-Salpêtrière, Service des Pathologies du Sommeil (Département R3S), Paris, France; ${ }^{4}$ Medical University, Department of Pulmology and Allergy,,Mother Teresa', Skopje, Mazedonia; ${ }^{5} \mathrm{UZ}$ Leuven/Campus Gasthuisberg, Slaapcentrum, Leuven, Belgien; ${ }^{6} \mathrm{Helsinki}$ Sleep Clinic, Vitalmed Research Centre, Helsinki, Finland; ${ }^{7}$ University of Turku, Sleep Research Unit Lemminkäisenkatu, Turku, Finland; ${ }^{8}$ Charité Universitätsmedizin Berlin, Interdisziplinäres Schlafmedizinisches Zentrum, Berlin, Germany; ${ }^{9}$ Alexandrovska Hospital Medical University, Department of Internal Medicine, Pulmonology, Sofia, Bulgaria; ${ }^{10} \mathrm{St}$. George Hospital Medical University, Department of Internal Medicine, Pulmonology, Plovdiv, Bulgaria; ${ }^{11}$ Grenoble University Hospital, University Hospital Thorax et Vaisseaux, Laboratoire EFCR et Sommeil, Grenoble, France; ${ }^{12}$ Bioprojet, Medical, Paris, France; ${ }^{13}$ Bioprojet Biotech, Saint Grégoire, France; ${ }^{14}$ Montpellier University Hospital, Unité des Troubles du sommeil et de l'éveil, Département de Neurologie, Montpellier, France

Objectives: Residual Excessive Daytime Sleepiness (rEDS) occurs in up to $12 \%$ of Obstructive Sleep Apnea (OSA) patients despite optimal continuous positive airway pressure (CPAP) therapy. This study assessed the efficacy and safety of pitolisant, a selective histamine H3-receptor antagonist waking agent in moderate to severe OSA treated by CPAP but exhibiting rEDS.

Methods: In a multicentre, double-blind, randomized, placebo-controlled trial, Pitolisant was individually titrated up to $20 \mathrm{mg}$ /day over 12 weeks. Primary endpoint was the Epworth Sleepiness Scale (ESS) score change in intention to treat. Key secondary endpoints were maintenance of wakefulness (Oxford Sleep Resistance Test (OSleR)), clinical global impressions of severity (CGI), patient's global opinion (PGO) and safety.

Results: 244 OSA patients (mean age: 53.1 years, mean Apnoea+hypopnea index under CPAP: 4.18/hour, baseline ESS score: $14.7( \pm 2.9))$ were randomized to Pitolisant $(n=183)$ and placebo $(\mathrm{pl})(n=61)$. ESS was significantly reduced with Pitolisant compared to $\mathrm{pl}-2.6$ (95\% CI: [-3.9; -1.4$]$ ) $(p<0.001)$. The ratio of increase in mean latency during OSleR Tests significantly improved with Pitolisant vs $\mathrm{pl}(1.44$ vs $1.22, p=0.05)$. The overall impact of Pitolisant was confirmed by physicians (CGI-C) and patients (PGO). Adverse event incidence was higher with Pitolisant vs pl (47.0\% and $32.8 \%, p=0.03$ ), the difference was mainly due to a higher proportion of headache, and insomnia. No cardiovascular or other significant safety concerns were reported.

Conclusions: Pitolisant as add-on to CPAP therapy for rEDS in OSA patients significantly reduced EDS and improved both patient and physician disease severity assessment.

Disclosure: The study was sponsored by bioprojet pharma. J. L. Pépin,

J. Verbraecken, V. Attali, D. Dokic, B. Buyse, M. Partinen, T. Saaresranta, I. Fietze, O. Georgiev, G. Belev, R. Tamisier, P. Levy, Y. Dauvilliers: clinical investigators of the study. I. Lecomte: employee of bioprojet pharma J.M. Lecomte, J.C. Schwartz: founder of bioprojet pharma.
Keywords: Pitolisant, Obstructive sleep apnea, Excessive daytime sleepiness, Positive airway pressure, Epworth Sleep Scale

\section{KV 22}

Long-term effects of solriamfetol on quality of life in participants with excessive daytime sleepiness associated with narcolepsy or obstructive sleep apnoea

A. Malhotra', J. L. Pépin'2, R. Schwab ${ }^{3}$, C. Shapiro 4 , J. Hedner', M. Ahmed N. Foldvary-Schaefer ${ }^{7}$, P.J. Strollo ${ }^{8},{ }^{*}$ G. Mayer, ${ }^{, 10}, K_{\text {K. Sarmiento }}{ }^{11}$, M. Baladi ${ }^{12}$, M. Bron ${ }^{12}$, P. Chandler ${ }^{12}$, L. Lee ${ }^{12}$, T.E. Weaver ${ }^{13}$

'Division of Pulmonary, Critical Care and Sleep Medicine, University of California San Diego Medical Center, La Jolla, United States; ${ }^{2}$ Grenoble Alpes University Hospital, Grenoble, France; ${ }^{3}$ University of Pennsylvania Medical Center, Philadelphia, United States; ${ }^{4}$ University of Toronto, Toronto, Canada; ${ }^{5}$ Sahlgrenska University Hospital, Gothenburg University, Gothenburg, Sweden; ${ }^{6}$ Cleveland Sleep Research Center, Cleveland, United States; ${ }^{7}$ Cleveland Clinic Lerner College of Medicine, Cleveland, United States; ${ }^{8}$ University of Pittsburgh/Veterans Administration Pittsburgh Health System, Pittsburgh, United States; ${ }^{\circ}$ Hephata Klinik, Schwalmstadt, Germany; ${ }^{10}$ Philipps University, Marburg, Germany; ${ }^{11}$ San Francisco Veterans Administration Healthcare System, San Francisco, United States; ${ }^{12} \mathrm{Jazz}$ Pharmaceuticals, Palo Alto, United States; ${ }^{13}$ College of Nursing, University of Illinois at Chicago, Biobehavioral Health Science, Chicago, United States

Objectives: Solriamfetol is a dopamine/norepinephrine reuptake inhibitor approved in the EU and US to treat excessive daytime sleepiness (EDS) associated with narcolepsy $(75-150 \mathrm{mg} / \mathrm{d})$ or obstructive sleep apnoea (OSA; $37.5-150 \mathrm{mg} / \mathrm{d})$. Solriamfetol demonstrated improvements on quality-oflife (QoL) measures in 12-week, placebo-controlled studies in participants with narcolepsy or OSA. The current study (NCT02348632) evaluated long-term effects (up to 52 weeks) of solriamfetol on QoL.

Methods: Participants from the US (85\%), EU (11\%), and Canada (4\%) with narcolepsy or OSA who completed previous solriamfetol studies were eligible for the long-term study (safety population, $\mathrm{N}=643$; narcolepsy, $n=226$; OSA, $n=417$ ). A 2-week titration period was followed by a maintenance phase of $\leq 50$ weeks (stable doses of 75,150 , or $300 \mathrm{mg} / \mathrm{d}$ ). Changes from baseline on the Functional Outcomes of Sleep Questionnaire short version (FOSQ-10), Work Productivity and Activity Impairment Questionnaire: Specific Health Problem (WPAI:SHP), and 36-Item Short Form Health Survey version 2 (SF-36v2) were summarised for the overall population and for the OSA and narcolepsy subgroups. No formal statistical testing was performed. Data are reported for participants who enrolled directly from one of the 12-week phase 3 solriamfetol trials; baseline refers to parent study baseline.

Results: Improvements in FOSQ-10 Total score (mean [SD] change of 3.7 [3.0]) were sustained for the duration of solriamfetol treatment; changes were similar between narcolepsy and OSA. On WPAI:SHP, mean reduction (improvement) was $\geq 25 \%$ for $\%$ activity impairment outside of work, \% impairment while working (presenteeism), and \% overall work impairment; results were generally similar for each subgroup. For SF-36v2, physical and mental component summary scores showed improvements in participants" health state (mean [SD] changes of 3.1 [6.9] and 4.3 [8.4], respectively); these improvements were maintained for the study duration. Common adverse events (AEs; $\geq 5 \%$ ) included headache, nausea, insomnia, nasopharyngitis, dry mouth, anxiety, decreased appetite, and upper respiratory tract infection, and were similar in narcolepsy and OSA; 27 participants $(4.2 \%)$ had $\geq 1$ serious AE.

Conclusions: Solriamfetol demonstrated sustained improvements on functionality and work productivity measures for up to 52 weeks in participants with EDS associated with narcolepsy or OSA. Safety was similar in the narcolepsy and OSA subgroups.

Keywords: JZP-110, Narcolepsy, OSA, Questionnaire, Clinical trial 


\section{KV 23}

\section{Effects of solriamfetol on 24-hour blood pressure patterns in participants with excessive daytime sleepiness associated with obstructive sleep apnoea}

*P. J. Strollo', A. Malhotra' ${ }^{2}$, K. Strohß', J. L. Pépin ${ }^{4}$, P. Schweitzer ${ }^{5}$,

G. J. Lammers ${ }^{6,7}$, J. Hedner ${ }^{8}$, M. Baladi ${ }^{9}$, L. Carter ${ }^{9}$, S. Bujanover ${ }^{9}$, Y. Dauvilliers ${ }^{10}$

'University of Pittsburgh/Veterans Administration Pittsburgh Health System, Pittsburgh, United States; ${ }^{2}$ University of California San Diego Medical Center, Division of Pulmonary, Critical Care and Sleep Medicine, San Diego, United States; ${ }^{3}$ Case Western Reserve University, Cleveland, United States; ${ }^{4}$ Grenoble Alpes University Hospital, Grenoble, France; ${ }^{5}$ Sleep Medicine and Research Center, St. Luke's Hospital, Chesterfield, United States; ${ }^{6}$ SleepWake Centre SEIN, Heemstede, Netherlands; ${ }^{7}$ Leiden University Medical Centre, Leiden, Netherlands; ${ }^{8}$ Sahlgrenska University Hospital, Gothenburg

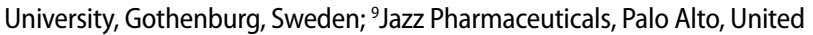
States; ${ }^{10} \mathrm{Gui}$-de-Chauliac Hospital, Chumontpellier, France

Objectives: Solriamfetol, a dopamine/norepinephrine reuptake inhibitor, is approved in the EU and US to improve wakefulness in adults with excessive daytime sleepiness associated with obstructive sleep apnoea (OSA; $37.5-150 \mathrm{mg} /$ day). Previous solriamfetol studies reported small mean increases in blood pressure (BP) in participants with OSA. These analyzes evaluated the time course of these effects and effects on nocturnal decreases in BP ("dipping"; "non-dipping" is a risk factor for adverse cardiovascular outcomes) in participants with OSA.

Methods: Twenty-four-hour ambulatory BP monitoring was conducted at baseline and Week 8 in a 12-week randomized controlled trial in participants treated with placebo or solriamfetol $(37.5,75,150$, or $300 \mathrm{mg} /$ day) (NCT02348606; EudraCT 2014-005514-31). Mean BP in 2-hour intervals across the day and the percentage of participants with non-dipping BP profiles were summarized (no formal statistical analyzes performed). Results: The safety population included 474 participants. At baseline, $50 \%$ (placebo) and $49 \%$ (combined solriamfetol) of participants had hypertension, and $51 \%$ and $49 \%$, respectively, used antihypertensive medications. At Week 8, mean increases in BP were observed in the 75 and $300 \mathrm{mg}$ groups from $6 \mathrm{AM}$ until $8 \mathrm{PM}$; increases in the 37.5 and $150 \mathrm{mg}$ groups were minimal. At baseline, $58 \%$ (placebo) and $55 \%$ (combined solriamfetol) of participants were non-dippers (defined as $<10 \%$ decrease in mean arterial pressure during sleep). There was no increase in the percentage of non-dippers at Week 8 relative to baseline (placebo, $56 \%$; combined solriamfetol, $53 \%$ ). Results were similar when dipping was defined by changes in mean systolic or diastolic BP. During the study, no placebo-treated participants and $1 \%$ of solriamfetol-treated participants initiated antihypertensive medication. One participant (receiving $150 \mathrm{mg}$ solriamfetol) discontinued due to an adverse event (AE) of increased BP.

Conclusions: At baseline, approximately half the study population had hypertension and/or used antihypertensive medications. Effects of solriamfetol on BP at the highest approved dose $(150 \mathrm{mg} /$ day $)$ were transient across the day. The percentage of participants with a non-dipping BP profile did not increase with solriamfetol at any dose studied. Few participants initiated new antihypertensive medication or discontinued due to AEs of hypertension/increased BP.

Keywords: JZP-110, Hypertension, Cardiovascular disease, Diastolic pressure, Systolic pressure

\section{KV 24}

A multi-center, two-stage, single-arm, prospective, first-night order cross-over, evaluator-blind study to evaluate the efficacy, safety and tolerance of the iNAP ${ }^{\oplus}$ sleep therapy system in adults with obstructive sleep apnea

${ }^{*}$ G. Nilius', M. Farid-Moayer', C. M. Lin', L. Knaack', Y.P. Wang ${ }^{5}$, D. Dellweg ${ }^{6}$, R. Stoohs ${ }^{7}$, J. H. Ficker ${ }^{8}$, W. J. Randerath ${ }^{9}$, M. B. Specht ${ }^{10}$, W. Galetke ${ }^{11}$, H. Schneider ${ }^{12}$

${ }^{1}$ Kliniken Essen-Mitte, Essen, Germany; ${ }^{2}$ PENINSULA SLEEP CENTER, Burlingame, United States; ${ }^{3}$ Shing-Kong Wu Ho-Su Memorial Hospital, Taipei City, Taiwan; ${ }^{4}$ INTERSOM Köln, Köln, Germany; ${ }^{5}$ Mackay Memorial Hospital, Taipei, Taiwan; ${ }^{6}$ Fachkrankenhaus Kloster Grafschaft GmbH, Schmallenberg, Germany; ${ }^{7}$ Somnolab Schlaflabor am, Essen, Germany; ${ }^{8}$ Klinikum Nürnberg, Nürnberg, Germany; ${ }^{9}$ Krankenhaus Bethanien gGmbH, Solingen, Germany; ${ }^{10}$ DKD Helios Klinik, Wiesbaden, Germany; ${ }^{11}$ HELIOS Klinik Hagen-Ambrock, Hagen, Germany; ${ }^{12}$ American Sleep Clinic Frankfurt, Frankfurt a. M., Germany

Objectives: Obstructive sleep apnea (OSA) occurs during sleep when the upper airway collapses during inspiration and remains closed despite continued inspiratory effort. Intraoral devices, with or without applied negative oral pressure, stabilize the oropharynx and have reduced the severity of OSA in selected patients. We are reporting results from a multi-center, prospective, first-night-order cross-over, study of the efficacy, safety and tolerance of the iNAP ${ }^{\circledR}$ Sleep Therapy System, a system that applies negative oral pressure.

Methods: One hundred thirty (130 patients) fulfilled all the inclusion criteria and none of the exclusion criteria and completed a baseline and 1st treatment polysomnographic study (Tx PSG). Sixty-three (63) patients, who had a Total Sleep Time (TST) $\geq 4 \mathrm{~h} /$ night on the Baseline PSG Study, an oral negative vacuum time maintained by iNAP ${ }^{\oplus} \geq 4 \mathrm{~h} /$ night $^{2}$ and TST $\geq 4 \mathrm{~h} /$ night (1st Tx PSG Study), formed the Primary Endpoint (PE) cohort, and 54 of 63 patients completed a 2nd Tx PSG at least 28 days after the 1st Tx PSG.

Results: Sixty-three adults (18 75 years, body-mass index $<33 \mathrm{~kg} / \mathrm{m} 2$, oxygen desaturation index between 10 60 events/hr, and apnea hypopnea index (AHI) between 15 55 events/hr) formed the PE cohort. Thirty-three (33) of these patients $(52 \%$; $95 \% \mathrm{CI}=40-65 \%, p<0.001)$ responded to iNAP Tx 1st PSG (had a $>50 \%$ reduction in AHI compared to baseline and treated AHI $\leq 20$ events/hr). The reduction in AHI was durable; a similar number of patients maintained a response to iNAP on the 2nd Tx PSG 28 days later. iNAP therapy was associated with a significant increase in the average oxy-hemoglobin saturation during sleep, and a significant reduction in the average percent desaturation associated with apneas and hypopneas and the total sleep time spent with an oxy-hemoglobin saturation below $90 \%$. The incidence of adverse events was low, and all adverse events were self-limited. Patients tolerated the iNAP device well and used the device on average $5.6 \mathrm{~h}$ per night during the 28 -day study period.

Conclusions: The iNAP ${ }^{\oplus}$ Sleep Therapy System achieved a significant, durable clinical effect in a significant fraction of selected OSA patients with moderate to severe OSA with an excellent comfort and safety profile. Thus, approximately half of appropriately selected patients with moderate to severe OSA may be expected to benefit from treatment with the iNAP ${ }^{\oplus}$ Sleep Therapy System.

Keywords: OSA, Oral negative pressure, Apnea hypopnea index, Therapy, Oxy-hemoglobin saturation

\section{KV 25}

Komorbide Insomnie bei chronischen Schmerzen: Schlaftraining in der orthopädischen Rehabilitation

*L. Feustel', L. Ende', C. Onderka'2, C. Franze ${ }^{3}$, N. Tscharaktschiew ${ }^{1}$

${ }^{1} T U$ Chemnitz, Institut für Psychologie - Allgemeine \& Biopsychologie,

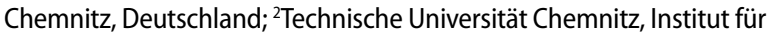
Psychologie, Chemnitz, Deutschland; ${ }^{3}$ Dr. Ebel Klinik Bad Brambach, Bad Brambach, Deutschland 
Fragestellung: Zwischen chronischen Schmerzen und Ein- und Durchschlafstörungen (Insomnien) besteht ein bidirektionaler Zusammenhang. Mangelnder Tiefschlaf kann sich negativ auf die Schmerzstärke auswirken und Schmerzen können die Schlafqualität reduzieren. Das Schlaftraining für Patienten mit chronischen Schmerzen und komorbider Insomnie basiert auf Methoden der kognitiven Verhaltenstherapie bei Insomnie (KVT-I; z. B. Schlafhygiene, Schlafrestriktion, kognitives Umstrukturieren) sowie der Psychologischen Schmerztherapie. Die Methoden werden gleichermaßen auf Schlaf und Schmerz angewendet. So wird u. a. ein Schlaf-Schmerz-Protokoll eingesetzt und der Zusammenhang zwischen Schlaftiefe und Schmerzwahrnehmung erörtert. Das Training wurde in drei Settings (ambulante Physiotherapie, ambulante orthopädische Rehaklinik, stationäre orthopädische Rehaklinik) auf seine Wirksamkeit geprüft. Patienten und Methoden: Die Stichprobe umfasst $N=53$ Patienten mit chronischen Schmerzen, mit einer Trainingsgruppe (TG) aus drei Settings (ambulante Physiotherapie: $n=12$, ambulante orthopädische Reha: $n=14$, stationäre orthopädische Reha: $n=9$ ) und einer gemeinsamen Kontrollgruppe (KG: $n=18$ ). Das Training besteht aus 4 Modulen mit einer Behandlungsdauer von 3 Wochen. Es wurden gemischte Varianzanalysen und $\mathrm{t}$-Tests berechnet.

Ergebnisse: Schlafqualität, Schlafeffizienz, Fokussing, maximale und durchschnittliche Schmerzstärke in der Nacht, durchschnittliche Schmerzstärke am Tag, schmerzbezogene Hilflosigkeit/Depression und Ängste verbesserten sich in der Gesamt-TG stärker als in der KG, mit Effekten von $\eta^{2}=0,01$ (Hilflosigkeit/Depression) bis 0,07 (Schlafqualität) für die Interaktion zwischen Zeit (prä vs. post) x Gruppe (TG vs. KG) (alle $p<0,05)$. Für die maximale Schmerzstärke am Tag und Grübeln fanden sich keine Interaktionseffekte. Die einzelnen TG erreichten signifikante Effekte von $\mathrm{d}=-0,62$ (max. Schmerzstärke Nacht, ambulante Reha) bis -2,96 (Schlafqualität, stationäre Reha). Für Fokussing (stationäre Reha, $\mathrm{d}=-0,57$ ) und maximale Schmerzstärke am Tag (ambulante Physiotherapie, $\mathrm{d}=-0,71$; ambulante Reha, $\mathrm{d}=-0,53)$ zeigten sich keine signifikanten Effekte.

Schlussfolgerungen: Das Schlaftraining wurde erfolgreich im ambulanten und stationären Bereich eingesetzt. Unterschiede der jeweiligen Effektstärken in den drei Settings werden diskutiert. Das Training sollte an größeren Stichproben weiter evaluiert werden.

Schlüsselwörter: Chronische Schmerzen, Schlaftraining, Insomnie, komorbid, KVT-I

\section{KV 26}

\section{Einfluss verschiedener PAP-Therapie-Versorgungsmodelle auf mittelfristige Therapieabbruchsraten}

Holger Woehrle', Joachim H. Ficker ${ }^{3,4}$, Andrea Graml', Juergen Schnepf ${ }^{2}$, Ingo Fietze $^{5}$, Peter Young ${ }^{6}$. Christoph Schoebel', Michael Arzt ${ }^{8}$

${ }^{1} S c h l a f-$ und Beatmungszentrum Blaubeuren, Lungenzentrum Ulm, Ulm, Deutschland; ${ }^{2}$ ResMed Science Center, Martinsried, Deutschland; ${ }^{3}$ Klinikum Nürnberg, Nürnberg, Deutschland; ${ }^{4}$ Paracelsus Medizinische Privatuniversität Nürnberg; ${ }^{5}$ Charité-Universitätsmedizin, Berlin, Deutschland; ${ }^{6}$ Medical Park, Abteilung für Neurologie, Bad Feilnbach, Deutschland; ${ }^{7}$ Pneumologie, Ruhrlandklinik, Universität Duisburg-Essen, Essen, Deutschland; ${ }^{8}$ Schlafmedizinisches Zentrum, Innere Medizin II, Regensburg, Deutschland

Hintergrund: Durch Telemonitoring (TM) unterstützte proaktive Patientenversorgung (Telemonitoring guided care (TM-GC (Airview))) bei Patienten mit Schlafapnoe (SA) mit positivem Atemwegsdruck (PAP) ist mit reduzierten Therapieabbruchraten innerhalb des ersten Behandlungsjahres assoziiert und der zusätzliche Einsatz eines Patienten-Engagement-Tools (PEng-Tool (MyAir)) verbessert die PAP-Nutzung innerhalb der ersten 180 Tage. Es liegen jedoch bisher keine Daten zu mittelfristigen PAP-Therapie-Abbruchraten vor.

Methoden: Die Daten eines deutschen Gesundheitsdienstleisters (ResMed Healthcare, Deutschland) wurden retrospektiv analysiert. Patienten, die zwischen 2004 und 2016 mit einer PAP-Therapie begonnen hatten, wurden eingeschlossen. Wir untersuchten die mittelfristigen Therapieab-

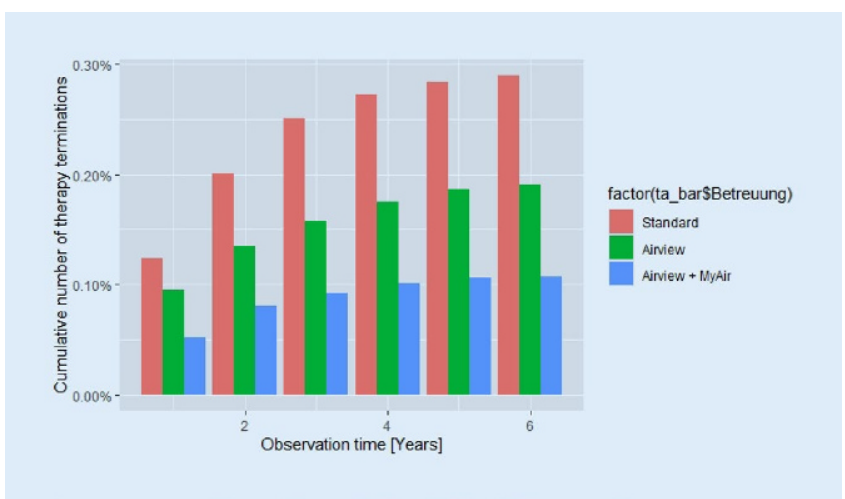

\section{Abb. $1 \mid$ KV 26 A}

bruchsraten bei Patienten, die Standardversorgung, TM-GC und zusätzlich ein Peng-Tool erhielten. Cox Proportional Hazard Modelle wurden entwickelt.

Ergebnisse: 69.444 Patienten wurden analysiert (Alter $63 \pm 13$, männlich $73 \%$, versichert $86 \%$, APAP+CPAP $90 \%$, Bilevel/ASV $10 \%$, Nasenmaske $49 \%$, Nasenpolstermaske $13 \%$ und Vollmaske $38 \%$ ). Die durchschnittliche Behandlungsdauer betrug 3,7 $\pm 1,8 \mathrm{~h}$ Jahre, die Therapieabbruchraten waren $29 \%$ bei Standardversorgung, $19 \%$ bei TM-GC und $11 \%$ bei PENg-Tool $(p<0,001)$. Die Dynamik der Therapieabbrüche sind in - Abb. 1 aufgeführt. Schlussfolgerungen: Die Standardversorgung ist mit deutlich höheren mittelfristigen PAP-Therapie-Abbruchraten im Vergleich zur proaktiven TM-GC assoziiert und der zusätzliche Einsatz eines PE-Werkzeugs ist mit einer weiteren Reduktion der Therapieabbruchraten assoziiert.

Unterstützt durch ResMed Germany Inc.

\section{Poster}

\section{P 1}

\section{Ohrnahe EEG-Sensoren aus flexiblen Leiterbahnen zur Schlafphasenbestimmung}

${ }^{*}$ C. F. da Silva Souto', *W. Pätzold', I. Wolf', M. Paul', I. Matthiesen ${ }^{3}$, M. G. Bleichner ${ }^{3}$, S. Debener ${ }^{3}$

'Fraunhofer-Institut für Digitale Medientechnologie IDMT, Institutsteil Hör-, Sprach- und Audiotechnologie HSA, Oldenburg, Deutschland; ${ }^{2}$ PSGAuswertungs-Service, Stadtlohn, Deutschland; ${ }^{3}$ Universität Oldenburg, Neuropsychology Lab, Oldenburg, Deutschland

Fragestellung: Eine komfortable, diskrete und robuste Erfassung des Schlaf-EEGs ist bisher schwierig zu realisieren. Wir untersuchen, wie gut gedruckte, flexible Elektroden für das Monitoring im heimischen Umfeld geeignet sind. Das ohrnahe cEEGrid (Debener et al., 2015; Bleichner \& Debener, 2017) ist für die Messung des Nachtschlafs im Labor bereits erprobt (Sterr, et al., 2018).

Patienten und Methoden: 10 Probanden wurden mit einem cEEGrid und einem tragbaren Verstärker (mBrainTrain, Serbien) ausgestattet. Zusätzlich wurde das EEG von Fpz, EOG1 und EOG2 aufgezeichnet. Die Datenübertragung erfolgte drahtlos auf ein Smartphone. Im Durchschnitt lieferte jeder Proband Daten über $M=7,48$ h Eine Schlafexpertin erstellte Hypnogramme und annotierte Grapho-Elemente gemäß AASM. Basierend auf diesen Annotationen wurden Signalausschnitte der konventionellen Elektroden mit denen der cEEGrids korreliert um optimale Elektrodenkonfigurationen zu ermitteln.

Ergebnisse: Der Schlafexpertin gelang es anhand des ohrnahen EEGs Hypnogramme zu erstellen. Erste Ergebnisse deuten hier auf eine beachtliche Übereinstimmung hin $(\kappa=0,79)$. Die Korrelationsanalyse der Grapho-Elemente ergab für die passendste Elektrodenkonfiguration des cEEGrids im 


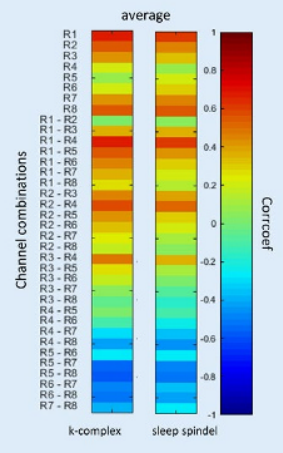

a

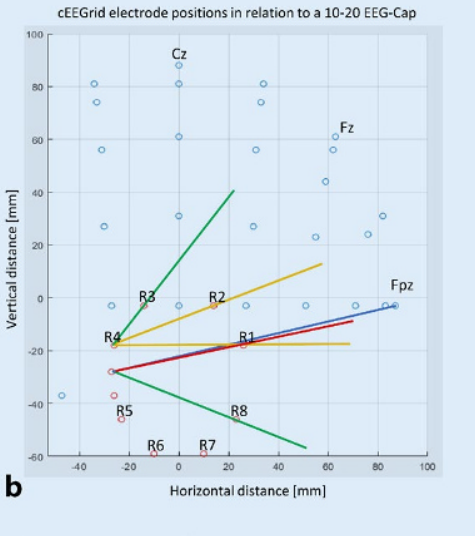

Abb. $1 \mid \mathrm{P} 1 \mathbf{4}$

Mittel einen Korrelationskoeffizienten von 0,65 (s. • Abb. 1a). Weiter zeigten die Ergebnisse, dass die Grapho-Elemente gut durch die frontal orientierten Elektrodenkombinationen repräsentiert werden ( $\bullet$ Abb. 1b). Schlussfolgerungen: Die Ergebnisse deuten darauf hin, dass auch ein reduziertes Elektrodenset ausreicht, um Schlafphasen, K-Komplexe und Spindeln zu detektieren. Dies eröffnet Entwicklungsmöglichkeiten in Richtung eines minimiertem, vom Nutzer selbst anzulegenden Systems mit hohem Tragekomfort.

Schlüsselwörter: Mobiles EEG, cEEGrid, Schlafphasenbestimmung, KKomplex, Schlafspindel

\section{Literatur}

1. Bleichner MG, Debener S (2017) Concealed, unobtrusive ear-centered EEG acquisition: CEEGrids for transparent EEG. Front Hum Neurosci 11:

2. Debener S, Emkes R, Vos MD, Bleichner M (2015) Unobtrusive ambulatory EEG using a smartphone and flexible printed electrodes around the ear. Sci Rep 5(1):

3. Sterr A et al (2018) Sleep EEG derived from behind-the-ear electrodes (CEEGrid) compared to standard polysomnography: A proof of concept study. Front Hum Neurosci 12:

\section{P 2}

Modulation of the neurothrophic activity by Ballota nigra $L$. Crataegus oxycantha L., Passiflora incarnata L. and Valeriana officinalis $L$. and their Combination in vitro

\section{A. Shcherbakova', C. Kolb², O. Kelber', G. Ulrich-Merzenich ${ }^{* 1}$}

'Universitätsklinikum Bonn, Medizinische Klinik III, AG Synergieforschung, Bonn, Germany; ${ }^{2}$ Steigerwald Arzneimittelwerk GmbH, R\&D

Phytomedicines Development Center, Consumer Health Medical \& Clinical Affairs Phytomedicine, 64295 Darmstadt, Germany

Objectives: Neurotrophins such as the brain derived neurotrophic factor (BDNF) as well as cytokines play an important role in neuroprotection, the control of the central nervous system, and change with different sleep patterns. We questioned whether the plant extracts of Ballota nigra $\mathrm{L}, \mathrm{Cra}$ taegus oxycantha L, Passiflora incarnata L. and Valeriana officinalis L. alone or in combination can modulate the pro-inflammatory cytokines, neurothrophines or neurotransmitters in vitro.

Methods: Synchronized human neuroblastoma SH-SY5Y cells were treated with the different aequeous plant extracts $(5-400 \mu \mathrm{g} / \mathrm{ml})$ alone and in combination $(1 / 1 / 4 / 1)$. As reference drug Lorazepam (LZ) was used. Resazurin was used to determine cell viability. TNF-alpha and IL-1ß were estimated in the supernatant by ELISA. An unbiased transcriptomic approach was used to determine the overall gene expressions induced by the different plant extracts/combination. Deepsequencing data were evaluated with different tools including Principal Component Analysis (PCA) and differential gene expressions.
Results: Neither the single plant extracts nor the combination or the reference drug LZ reduced the cell viability in the tested concentrations. The treatments did not stimulate TNF-a or IL-1b releases. The gene expression profiles of the four plant extracts, their combination and LZ could be clearly distinguished in the PCA-Analyses. The subsequent detailed gene expression analyses revealed that cytokines, neurotrophins (e. g. BDNF) and neurotransmitters were among the 2.500 genes with the most variable expression. The four plant extracts and the combination exhibited different expression profiles, also in comparison to LZ.

Conclusions: The four plant extracts and their combination target in $\mathrm{SH}-$ SY5Y cells relevant genes for the modulation of the neurotrophic activity. Keywords: Sleep, Neurotrophines, Phytomedicine, SH-SY5Y Cells, Geneexpression-Analyses

\section{P 3}

Aktivierung dilatierender Muskeln des oberen Atemwegs durch Elektrostimulation des Ramus internus des Nervus laryngeus superior als potentieller Therapieansatz bei obstruktiver Schlafapnoe - Vorläufige Ergebnisse

\section{*J. T. Maurer}

Klinik für Hals-Nasen-Ohrenheilkunde, Kopf- und Hals-Chirurgie, Universitätsmedizin Mannheim, Sektion Schlafmedizinisches, Mannheim, Deutschland

Fragestellung: Die Verbindung von reduzierter Genioglossus (GG) Muskelaktivität und dem Auftreten einer obstruktiven Schlafapnoe (OSA) ist gut belegt. In einer Studie mit 18 Hunden konnte eine erhöhte GG Aktivität nach Stimulation des R. internus des N. laryngeus superior (iSLN) beobachtet werden. Das Ziel dieser klinischen Studie ist, zu bestimmen, ob die direkte iSLN Elektrostimulation die Aktivität des GG und weiterer Muskeln zur Öffnung des oberen Atemwegs auch beim Menschen erhöhen kann.

Patienten und Methoden: 10 Patienten, bei denen im Zuge einer Laryngektomie unter Sevofluran-Anästhesie eine zumindest unilaterale Freilegung des iSLN durchgeführt werden kann, werden in die Studie eingeschlossen. Mittels einer bipolaren Sonde wird der freigelegte iSLN einseitig stimuliert. Die Muskelaktivität des ipsi- und kontralateralen GG und des Palatoglossus wird anhand von Elektromyografie-Aufzeichnungen bewertet.

Ergebnisse: Erste Ergebnisse von 3 Patienten zeigten, dass die unilaterale iSLN Stimulation bei $40 \mathrm{~Hz}$ mit Stimulationsströmen höher als 3,6 mA eine spezifische tonische EMG-Aktivität des ipsilateralen GG auslösen konnte. Die EMG-Aktivität hielt über die Stimulationsdauer hinaus an bis zu einer maximalen Dauer von 66,3 s. Es wurde auch eine erhöhte Aktivität des ipsilateralen Palatoglossus beobachtet.

Schlussfolgerungen: Erste Ergebnisse lassen vermuten, dass die Elektrostimulation des iSLN eine spezifische Reaktion des GG hervorrufen kann. Bei Anwendung an schlafenden OSA Patienten könnte dadurch eine ausreichende Stabilität der Atemwege im Rachen erzielt werden, um dem krankheitstypischen Kollaps der oberen Atemwege effizient entgegenzuwirken.

Schlüsselwörter: Neurostimulation, Obstruktive Schlafapnoe, Afferente Stimulation, Muskeltonus, EMG

\section{P 5}

\section{Auswirkungen von Motivation auf den Pupillenunruheindex unter} Berücksichtigung des Geschlechts

${ }^{*}$ T. Eggert, S. Mazzini, M. Götsch, L. Weigt, M. Guenther, K. Koss, K. Schmieder, H. Dorn, C. Sauter, H. Danker-Hopfe

Charité - Universitätsmedizin Berlin, Kompetenzzentrum Schlafmedizin, CC15, Berlin, Deutschland

Fragestellung: Eine Zwischenauswertung hatte ergeben, dass der Pupillenunruheindex (PUI) als Hauptergebnisparameter des Pupillographischen Schläfrigkeitstests (PST) motivationalen Aspekten zu unterliegen 
scheint. Dabei zeigte sich, dass der PUI willentlich in Richtung eines höheren Schläfrigkeitsgrades manipuliert werden konnte. In der vorliegenden Auswertung wurde überprüft, ob dieser Befund in der Gesamtstichprobe weiterhin Bestand hat und ob dieser Effekt möglicherweise geschlechtsspezifisch ist.

Patienten und Methoden: Insgesamt haben 50 Studienteilnehmer/Innen (10 Frauen) im Alter zwischen 18 und 30 Jahren die Studie komplett durchlaufen. Unter anderem umfasste die Studie drei Termine unter kontrolliertem Schlafentzug [ein Kontrollschlafentzug (K-SE) gefolgt von zwei Experimentalschlafentzügen mit den Motivationsbedingungen „Wachheit wird belohnt“ (W-SE) oder „Schläfrigkeit wird belohnt“ (S-SE)]. An allen drei Terminen erfolgten sowohl am Abend direkt nach dem Eintreffen im Schlaflabor als auch am Morgen nach dem Schlafentzug zu individuell konstant gehaltenen Zeitpunkten 11-minütige PST-Messungen. Schlafentzugsbedingte Veränderungen des PUI unter Baselinebedingung aber auch unter Berücksichtigung einer in Aussicht gestellten finanziellen Belohnung wurden für Männer und Frauen getrennt mittels Wilcoxon-VorzeichenRang-Test auf statistische Signifikanz geprüft.

Ergebnisse: Für beide Geschlechter konnte nach dem K-SE im Mittel (Median) eine statistisch signifikante Zunahme des PUI über Nacht beobachtet werden $\left(\delta^{\Uparrow}: 2,27, \mathrm{IQR}:-0,62,4,57, p=0,0086\right.$; 우 2,34 , IQR: 1,07, 3,17, $p=0,0039$ ). Während der PUI auch nach dem S-SE für beide Geschlechter am Morgen durchschnittlich signifikant größer als am Abend war ( $\delta$ : 3,80, IQR: $1,67,8,50, p<0,0001$; + : 3,41, IQR: $2,46,6,04, p=0,0059)$, erwies sich die PUI-Veränderung über Nacht nach dem W-SE nur bei den Männern als statistisch signifikant ( $\delta: 1,80, \mathrm{IQR}:-0,77,4,52, p=0,0059$; ; : $0,29$, IQR: $-1,21,4,86, p=0,6250)$. Ein statistisch signifikanter Unterschied zwischen den Schlafentzugsbedingungen konnte bei den Männern nur für den Vergleich S-SE vs. K-SE ( $p=0,0053$; W-SE vs. K-SE: $p=0,7013$ ) beobachtet werden. Bei den Frauen konnten dagegen keine Unterschiede zwischen den Schlafentzugsbedingungen festgestellt werden (S-SE vs. K-SE: $p=0,3223$; W-SE vs. K-SE: $p=0,6953$ ).

Schlussfolgerungen: Für das männliche Geschlecht konnte der Befund aus der Zwischenauswertung hinsichtlich einer motivationalen Beeinflussung des PUI bestätigt werden. Für die Teilstichprobe der Frauen ließ sich dieser Effekt hingegen nicht beobachten, was in der geringen Fallzahl begründet liegen könnte.

Schlüsselwörter: PST, PUI, Tagesschläfrigkeit, Motivation, Schlafentzug

\section{P 6}

\section{Studium und Mutterschaft = Doppelbelastung? - Gruppenvergleiche hinsichtlich Stress, Schlaf, psychischer Gesundheit und Coping}

*J. Faber, D. Hertel, A. A. Schlarb

Universität Bielefeld, Klinische Psychologie und Psychotherapie des Kindesund Jugendalters, Bielefeld, Deutschland

Fragestellung: Die Zahl der Studierenden, die während des Studiums ein Kind erwarten bzw. bereits ein Kind haben, ist in den letzten Jahren angestiegen. Dabei zeigen Studien in beiden Bereichen, Studium und Mutterschaft, ein erhöhtes Stresslevel und vermehrte Schlafprobleme. Das Ziel dieser Studie ist die Untersuchung, inwieweit eine Doppelbelastung bei Studierenden vorherrscht, die ein Kind erwarten bzw. ein Kind betreuen. Patienten und Methoden: In einer Onlineumfrage wurden weibliche Studierende in Deutschland mittels Fragebögen zu Stress, Schlaf, psychischer Gesundheit (Depression, Angst) und ihren Copingstrategien befragt. Bisher haben 395 Studierende an der Umfrage teilgenommen. Davon gaben 12 Personen an momentan schwanger zu sein, 84 Personen gaben an Kinder zu haben und 299 Personen ordneten sich der Gruppe der Studierenden ohne Kinder zu.

Ergebnisse: Zunächst sollen die Gruppen (Studierende ohne Kinder, schwangere Studierende und Studierende mit Kindern) auf den genannten Variablen verglichen werden. Zudem sollen die Zusammenhänge von Stress, Schlafqualität, depressiver und ängstlicher Symptome, sowie Coping für die verschiedenen Gruppen dargestellt werden.
Schlussfolgerungen: Der Einfluss von Stress, Schlaf und angewandten Copingstrategien, sowie der Vergleich mehrerer Studierendengruppen ermöglicht eine differenziertere Integration in präventive und beratende Maßnahmen, um die Gesundheit von Studierenden langfristig zu verbessern und die Abbruchquote des Studiums zu verringern.

Schlüsselwörter: Stress, Schlaf, Psychische Gesundheit, Studierende, Mutterschaft

\section{P 7}

\section{Neurophysiologische Auswirkungen obstruktiver Schlafapnoe:} ein Review

\section{K. Tziridis, C. Fischer, M. Traxdorf, *H. Schulze}

Friedrich-Alexander University Erlangen-Nürnberg (FAU), Department of

Otorhinolaryngology, Head and Neck Surgery, Erlangen, Deutschland

Fragestellung: Ziel der vorliegenden Arbeit war es, einen Überblick über neurophysiologische Auswirkungen, Pathogenese und klinische Manifestationen obstruktiver Schlafapnoe (OSA) zu geben.

Patienten und Methoden: Es wurde eine Datenbankrecherche auf den Plattformen PUBMED sowie GOOGLE SCHOLAR (Stand 01.06.2018) durchgeführt, sowie Fachinformationen schlafmedizinischer Gesellschaften (DGSM, AASM) und der Cochrane Collaboaration einbezogen. Insgesamt wurden 222 Studien und 6 Fachinformationen einbezogen. Alle Studien wurden anhand eines nach den Empfehlungen des Cochrane Instituts zur Literaturbewertung selbst erstellten Punktesystems nach folgenden Kriterien bewertet: 1) Größe des Patientenkollektivs, 2) Größe der Kontrollgruppe, Abstimmung der Fälle und Kontrollen auf 3) Alter, 4) Geschlecht und 5) Body Mass Index, Polysomnographie zur 6) OSA-Diagnose bei Patienten bzw. zum 7) OSA-Ausschluss bei Kontrollen, Ausschluss von Studienteilnehmern mit 8) potentiell beeinflussenden Begleiterkrankungen und 9) Medikation und 10) nur Einschluss von Studienteilnehmern, die noch keine OSA-Therapie erhalten haben.

Ergebnisse: Hauptursache zerebraler Anomalitäten und neurophysiologischer Defizite bei OSA-Patienten sind intermittierende Hypoxie und Schlaffragmentierung.

Beide Faktoren führen über molekulare und zelluläre Signalwege zu anatomisch-strukturellen, funktionellen, biochemisch-metabolischen und elektrokortikalen Veränderungen im Gehirn, zB lokale Volumenveränderungen, lokal veränderte Metabolitkonzentrationen, anomale Aktivierung und Vernetzung bestimmter Hirnregionen, Abweichungen in EEGRhythmus und Schlafarchitektur.

Hieraus resultiert als Hauptsymptom die extreme Tagesschläfrigkeit, daneben Störungen der Sinneswahrnehmung sowie Assoziationen mit Alzheimer, Parkinson, Epilepsie, Kopfschmerzen, Depressionen sowie kognitive Defizite.

Schlussfolgerungen: OSA kann zu weitreichenden neurophysiologischen Auswirkungen auf molekularer und zellulärer Ebene, als auch struktureller, funktioneller, metabolischer und elektrokortikaler Ebene der Betroffenen führen. haben. Daher ist eine frühzeitige Behandlung der kausal zugrundeliegenden intermittierenden Hypoxie und Schlaffragmentierung on essenzieller Bedeutung.

\section{P 8}

\section{Circadian sensitivity of hearing thresholds and tinnitus development to noise trauma in the Mongolian gerbil-an update}

\section{*K. Tziridis, J. Grimm, M. Traxdorf, H. Schulze}

Universitätsklinikum Erlangen, HNO-Klinik, Kopf- und Halschirurgie, Erlangen, Germany

Objectives: Hearing is essential for communication in humans as well as other social mammals like mice or gerbils. Hearing loss cannot only lead to social deprivation but may also cause co-morbidities like tinnitus, an auditory phantom percept of a sound without physical source. It is known that noise induced hearing loss has a circadian component: In mice, hearing thresholds are significantly stronger affected when the acoustic trauma 
is given during the night compared to rather mild effects on hearing when the trauma is applied during daytime. Last year we reported different circadian effects of an acoustic trauma in gerbils when it was applied during different time of day, now we added the effects during nighttime trauma. Methods: We investigate the effect of acoustic traumata $(2 \mathrm{kHz}, 115 \mathrm{~dB}$ SPL, $75 \mathrm{~min}$ ) applied either at $9 \mathrm{AM}, 1 \mathrm{PM}, 5 \mathrm{PM}$ or $12 \mathrm{PM}$ on the hearing thresholds and tinnitus development of 31 male Mongolian gerbils (Meriones unguiculatus). Hearing thresholds were measured by acoustic brainstem response (ABR) audiometry at $10 \mathrm{AM}$ one day before and one week after the trauma and possible tinnitus percepts were behaviorally assessed by the gap prepulse inhibition of the acoustic startle (GPIAS) at 9 AM one day before and one week after the trauma.

Results: We find daytime dependent changes of the trauma on mean hearing thresholds in a frequency dependent manner by one- and two-factorial ANOVAs with the maximum hearing loss (HL) one octave above the trauma frequency $(\mathrm{F}(3,104)=3.42, p=0.02)$, and-comparable to the results in mice-significant time dependent effects at the trauma frequency. We find a minimal $\mathrm{HL}$ after the $9 \mathrm{AM}$ trauma and the maximal $\mathrm{HL}$ after the $5 \mathrm{PM}$ and the $12 \mathrm{PM}$ traumata $(\mathrm{F}(6,46)=2.79, p=0.02)$. Additionally to the HL effects we find correlations of these threshold changes with the strength of the tinnitus percept (effect size of GPIAS) in the 5 PM trauma group $(p=0.02)$ and a trend in the $12 \mathrm{PM}$ trauma group $(p=0.07)$, i. e., animals with stronger tinnitus percepts showing better hearing thresholds than animals with light or no phantom percepts.

Conclusions: Taken together, also in gerbils circadian sensitivity of the hearing thresholds to noise trauma can be found and tinnitus strength correlates with it only when the trauma is applied at the most sensitive time, namely in the evening and night.

Keywords: Grundlagenforschung, Nager, Verhaltensmessungen, BERA, Hörsystem

\section{P 9}

\section{Eine Subgruppenanalyse zu den Unterschieden in polysomnographischen Parametern zwischen Schicht- und Tagarbeitenden mit Insomnie.}

\section{*J. Ell, B. Feige, D. Riemann, K. Spiegelhalder \\ Universitätsklinik Freiburg, Psychiatrie und Psychotherapie, Freiburg, Deutschland}

Fragestellung: Schichtarbeiter/innen leiden häufig unter insomnischen Symptomen. Dabei berichten sie subjektiv ähnliche Schlafparameter wie Arbeitnehmer/innen mit Insomnie, welche nicht in Schichtarbeit tätig sind. Um ein umfassendes Bild der Insomnie im Kontext Schichtarbeit zu erhalten, wurden in dieser Studie objektive Schlafparameter von Schichtarbeitenden mit denen von Tagarbeitenden verglichen.

Patienten und Methoden: Für die Analyse wurden Daten aus dem Zeitraum zwischen 1999 und 2020 des Schlaflabors der Abteilung Psychiatrie und Psychotherapie am Universitätsklinikum Freiburg gesichtet. In die Analyse wurden Personen eingeschlossen, welche die Kriterien einer Insomnie nach DSM-5 Kriterien erfüllten, keine den Schlaf beeinflussende Medikamente einnahmen sowie an keiner komorbiden psychischen oder körperlichen Erkrankung litten. Die polysomnographischen Daten von 11 Nachtschichtarbeitenden ( $18,2 \%$ weiblich; $\mathrm{M}_{\text {Alter }}=40,7$ Jahre $)$ und 12 Schichtarbeitenden ohne Nachtschicht (50,0\% weiblich; $\mathrm{M}_{\text {Alter }}=42,9 \mathrm{Jah}-$ re) wurden erfasst und mit den Daten von 23 Tagarbeitenden (gematcht für Alter und Geschlecht) verglichen. Hierzu wurden multivariate ANCOVAs mit der Kontrollvariable Geschlecht berechnet.

Ergebnisse: Es konnten keine signifikanten Unterschiede zwischen den Gruppen bezüglich ihrer Schlafkontinuität gefunden werden. Die Gruppen unterschieden sich somit nicht in der Dauer ihres Schlafes $\left(\mathrm{F}_{2,42}=1,46\right.$, $p=0,244)$, der Schlafeffizienz $\left(\mathrm{F}_{2,42}=1,27, p=0,304\right)$, der Einschlaflatenz $\left(\mathrm{F}_{2,42}=0,64, p=0,530\right)$, der Häufigkeit des Aufwachens während der Nacht $\left(\mathrm{F}_{2,42}=0,64 ; p=0,531\right)$, der Dauer der Wachphasen nach dem Einschlafen $\left(\mathrm{F}_{2,42}=1,36, p=0,267\right)$ sowie im Arousal Index $\left(\mathrm{F}_{2,40}=2,26 ; p=0,117\right)$. Auch bei Betrachtung der Schlafarchitektur zeigten sich keine signifikanten Unterschiede bei gleichen Anteilen von N1 $\left(\mathrm{F}_{2,42}=0,35 ; p=0,710\right), \mathrm{N} 2$
$\left(\mathrm{F}_{2,42}=1,06 ; p=0,355\right), \mathrm{N} 3\left(\mathrm{~F}_{2,42}=0,81 ; p=0,450\right)$ und REM $\left(\mathrm{F}_{2,42}=0,83\right.$; $p=0,443)$. Ferner unterschieden sich die Gruppen nicht signifikant in ihrer Schlafwahrnehmung, also der Differenz zwischen objektiv gemessenen und der subjektiv erlebten Schlafdauer $\left(\mathrm{F}_{2,37}=1,30 ; p=0,286\right)$.

Schlussfolgerungen: Schichtarbeitende mit Insomnie scheinen ein ähnliches polysomnographisches Schlafprofil aufzuweisen wie Tagarbeitende mit Insomnie. Zukünftige Forschungsarbeiten sollten mögliche Effekte der Methode der Wahl zur Behandlung der Insomnie, der kognitiven Verhaltenstherapie, auch bei Schichtarbeitenden systematisch untersuchen. Schlüsselwörter: Schichtarbeit, Insomnie, Polysomnographie, Schlaflabor, Schlafprofil

\section{P 10}

Null Bock auf meine Arbeit! - Der Zusammenhang von Schlafqualität, innerer Kündigung und dem Arbeitsverhalten, der Gesundheit und Lebenszufriedenheit

\section{*J. Faber, A. Czellnik, A. Meyer, J. Schwarze, A. A. Schlarb}

Universität Bielefeld, Klinische Psychologie und Psychotherapie des Kindesund Jugendalters, Bielefeld, Deutschland

Fragestellung: Die innere Kündigung ist ein Zustand der kognitiven, emotionalen und behavioralen Distanzierung von der Arbeit, der sich v.a. durch mangelnde Eigeninitiative und Interesse auszeichnet. Forschungsergebnisse zu dem Konstrukt der inneren Kündigung liegen nur vereinzelt vor, da man sich bei der Untersuchung von Zusammenhängen mit gesundheitlichen Problemen vermehrt auf die Arbeitszufriedenheit und Burnout fokussiert hat. Ziel dieser Studie war es daher, den aktuellen Forschungsstand hinsichtlich der inneren Kündigung und ihren Zusammenhängen mit der Schlafqualität, depressiver Symptomatik, somatischer Beschwerden, Stress, Ressourcen und Lebensqualität zu erweitern.

Fragestellung: In einer Onlineumfrage wurden nicht-selbstständige ArbeitnehmerInnen befragt, die mindestens $17,5 \mathrm{~h}$ pro Woche arbeiteten. Die TeilnehmerInnen ordneten sich basierend auf einer vorgegebenen Definition entweder der Gruppe der innerlich Gekündigten oder der nicht innerlich Gekündigten zu. Die obengenannten Konstrukte wurden mittels Fragebögen erfasst. Die Stichprobe umfasste 252 ArbeitnehmerInnen (weiblich $=174$, männlich $=76$, divers $=2$ ), wobei sich 52 Personen der Gruppe der innerlich Gekündigten und 200 Personen der Gruppe der nicht innerlich Gekündigten zuordneten.

Ergebnisse: Personen, die innerlich gekündigt hatten, berichteten über eine signifikant geringere Schlafqualität, mehr depressive Symptome, mehr somatische Beschwerden, mehr Stress und eine geringere Lebenszufriedenheit. Darüber hinaus zeigten die Selbstauskünfte signifikant geringere Level an Hilfsbereitschaft, Gewissenhaftigkeit, Unkompliziertheit und Eigeninitiative im Vergleich zu den Personen, die nicht innerlich gekündigt hatten. Ein signifikanter Unterschied bzgl. des Arbeitsverhaltens konnte nicht gezeigt werden.

Schlussfolgerungen: Die Studienergebnisse zeigen, dass auch hinsichtlich der Schlafqualität die Thematik der inneren Kündigung von hoher Relevanz ist, da jeder Fünfte der Befragten betroffen ist und erhebliche psychische und physische Beeinträchtigungen berichtet werden. Die Erkenntnisse können von ArbeitnehmerInnen als auch Unternehmen genutzt werden, um die Sensibilisierung und Relevanz präventiver Maßnahmen zu betonen.

Schlüsselwörter: Innere Kündigung, Schlaf, Depression, Stress, Lebensqualität

\section{P 11}

Zusammenhänge zwischen Schlafhygienewissen, Schlafhygiene und Schlafqualität in Abhängigkeit von Nachtschicht - eine

\section{Querschnittstudie}

${ }^{*}$ B. Klofat ${ }^{1,2}$, P. Gerlach ${ }^{1}$

${ }^{1}$ Hochschule Fresenius, Psychologie, Hamburg, Deutschland; ${ }^{2}$ Heinrich-

Heine-Universität, Klinische Psychologie, Düsseldorf, Deutschland 


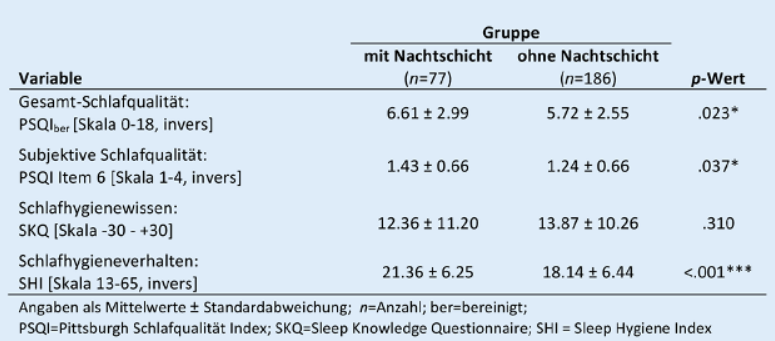

Abb. 1 | P 11 ॥ Mittelwertvergleiche (t-Tests) der Subgruppen bzgl. Schlafqualität, Schlafwissen, Schlafhygiene

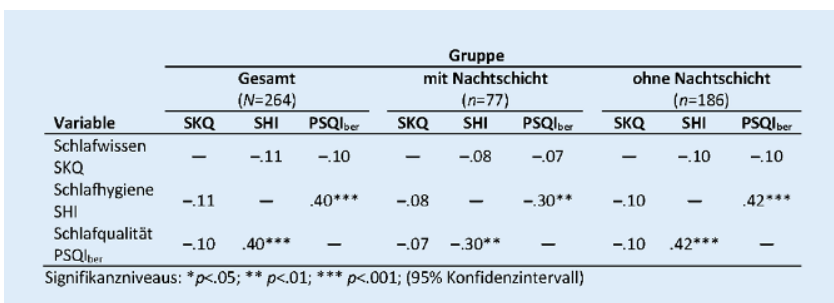

Abb. 2 |P 11 A Pearson Produkt-Moment-Korrelationen zwischen Schlafvariablen nach (Sub-)Gruppe

Fragestellung: Nachtschichtarbeitern werden Schulungen zu Schlafhygiene (SH) empfohlen, obwohl Zusammenhänge von Schlafhygienewissen (SHW), Schlafhygieneverhalten (SHV) und Schlafqualität (SQ) bisher wenig erforscht sind. Zwar moderiert SHV den Effekt von SHW auf SQ, SHW und SHV hängen aber bei schlechten Schlaf nicht unbedingt zusammen. Ziel der Studie war, diese Zusammenhänge in Abhängigkeit von Nachtdienst (ND) zu beleuchten.

Patienten und Methoden: Hierzu wurden in einer Querschnittstudie Berufstätige mit $(N=77)$ und ohne $(N=186)$ Nachtschichtdienst online mittels SKQ (Sleep Knowledge Questionnaire), SHI (Sleep Hygiene Index) und PSQI (Pittsburgh Schlafqualität Index) befragt.

Ergebnisse: Probanden (Pbn) mit ND beurteilten die SQ schlechter als Pbn ohne ND (oND). Der PSQI wurde um die Skala Schlafeffizienz bereinigt, die bei Tagschlaf nicht valide war. Es zeigt sich eine schlechtere SQ bei ND im Vergleich zu oND. Im SKQ ergab sich kein Unterschied, jedoch zeigt sich eine geringere Umsetzung von SHV bei Pbn mit ND (• Abb. 1). Nur in der Gesamtstichprobe korrelieren SHW und SHV schwach marginal signifikant, ebenso SKQ und PSQI. SHI und PSQI korrelieren mittelstark. Für ND-Pbn zeigt sich weder ein Zusammenhang zwischen SKQ und SHI, noch SKQ und PSQI. SHI und PSQI korrelieren moderat (- Abb. 2).

Nur $4 \%$ der PBn mit ND gaben an, vom Arbeitgeber schlafbezogene Informationsangebote erhalten zu haben.

Schlussfolgerungen: Trotz Limitationen bestätigt sich der negative Einfluss von ND auf SHV und SQ.

SHW scheint allenfalls marginal mit SHV und SQ zu korrelieren. Gesundheitsverhalten hängt von Wissen, aber auch Selbstwirksamkeitserwartung ab, die bei ND bzgl. SH gering sein kann. Regeln wie Rhythmik, Aktivitätsreduktion, Koffeinkarenz, leise Schlafumgebung sind bei ND kaum umsetzbar.

Die moderate Korrelation zwischen SHV und SQ korrespondiert mit anderen Studien und erklärt sich über multiple Einflußfaktoren, die nur z.T. beeinflußbar sind. Dass sich über Schulungen SHW verbessern lässt, bei ND aber nicht SQ, fanden auch andere Studien.

Angesichts Arbeitgeberfürsorgepflicht erstaunt, dass nur $4 \%$ der ND-Pbn Informationsangebote bejahten.

Wenn SHW allenfalls marginal zu SQ beiträgt, stellt sich die Frage, ob SHSchulungen im Kontext von ND sinnig sind. Aus gesundheitspsychologi- scher Sicht sollte ohnehin Verhältnisprävention Vorrang haben z. B. Hinterfragen von ND-Systemen oder NDe passend zum Chronotyp. Schlüsselwörter: Schlafhygiene, Nachtschicht, Schlafqualtät, Schlafwissen, Schlafverhalten

\section{P 12}

\section{Weiterentwicklung und Anwendung eines normbasierten Index zur Messung objektiver Schlafqualität}

\section{*T. A. Thomas, F. E. Gümüsdagli, R. Pietrowsky}

Heinrich-Heine-Universität Düsseldorf, Abteilung für Klinische Psychologie, Düsseldorf, Deutschland

Fragestellung: Eine Messung objektiver Schlafqualität in Forschung und Praxis erfolgt zumeist über die Betrachtung objektiver Schlafparameter. Thomas (2019) hat dazu einen Index entwickelt, der ökonomisch die Abweichung von altersnormwertiger Schlafqualität erfassen kann. Für jeden Schlafparameter wird eine eigene Kategorisierung mit einem Wertebereich von 1-10 relativ zu einem Normwert der entsprechenden Altersgruppe gebildet. Basierend darauf wird ein Summenwert für positive (PAS; N3 $\%$, REM \%, TST, SE \%) und negative (NAS; N1 \%, N2 \%, SL, Wachdauer) Schlafqualität gebildet und daraus ein Quotient (Index) berechnet. Es wird geprüft, ob dadurch eine Differenzierung zwischen Patienten mit obstruktivem Schlafapnoe-Syndrom (OSAS) und Gesunden erfolgen kann. Patienten und Methoden: Zur Anwendung des Index wurden aus der repräsentativen Stichprobe von Roizenblatt et al. (2015) randomisiert 313 Personen (57\% weiblich) mit und ohne OSAS ausgewählt. Die errechneten Scores für Patienten mit OSAS und Gesunden wurden verglichen. Ergebnisse: OSAS-Patienten $(M=21,66, S D=5,15)$ weisen eine höhere Ausprägung im NAS-Summenscore auf als Gesunde $(M=20,06$, $S D=5,07), t(311)=2,66, p=0,008, d=0,31$. Unter den etablierten Maßen der Schlafqualität war nur die Wachdauer nach Einschlafen bei OSASPatienten $(M=65,84, S D=46,79$ min) länger als bei Gesunden $(M=54,51$, $S D=44,49 \mathrm{~min}), t(311)=2,12, p=0,035, d=0,25$. Dabei ist die Effektstärke des NAS-Summenscores größer als für jeden anderen der in ihn einfließenden Schlafparameter selbst.

Schlussfolgerungen: Der weiterentwickelte Index wurde auf eine repräsentative Stichprobe angewendet und konnte besser zwischen OSAS-Patienten und Gesunden differenzieren als etablierte Maße, bei denen sich nur ein Parameter unterschieden hat. Der Vorteil kann dabei auf die globale Erfassung der Schlafqualität im NAS-Score zurückgeführt werden. Auf Basis der Ergebnisse werden Erweiterungen des Index wie um Mikrostrukturvariablen des Schlafs geprüft, um den Index auch für andere Anwendungen zu verbessern.

Schlüsselwörter: Schlafqualität, OSAS, Polysomnographie, Schlafindex, Schlafdiagnostik

\section{Literatur}

1. Roizenblatt S, Souza AL, Palombini L, Godoy LM, Tufik S, Bittencourt LRA (2015) Musculoskeletal pain as a marker of health quality. Findings from the epidemiological sleep study among the adult population of Sao Paulo city. PLOS ONE 10(11):e142726

2. Thomas, Tobias A. (2019). Entwicklung eines normbasierten Index zur Messung der objektiven Schlafqualität. Poster auf der Jahrestagung der Nordrhein-Westfälischen Gesellschaft für Schlafmedizin, Essen.

\section{P 13}

\section{Die Rolle der strukturierten Befunderhebung in der Diagnostik} und Therapieplanung schlafbezogener Atmungsstörungen

*K. Bahr, H. Gouveris, B. Ernst

Universitätsmedizin Mainz, Hals-, Nasen-, Ohrenklinik und Poliklinik, Mainz, Deutschland

Fragestellung: In der Diagnostik schlafbezogener Atemstörungen ist eine ausführliche Anamnese sowie Befunderhebung der anatomischen Besonderheiten der Kopf-Hals-Region für die Einleitung einer adäquaten Dia- 
gnostik und Therapie unabdingbar. Schlafmedizinische Anamnese und Befunderhebung haben sich in der Vergangenheit in ihrer Durchführung und Dokumentation als äußert zeitaufwändig herausgestellt. Die Verwendung einer strukturierten Befunderfassung (SR) von diagnostischen und therapeutischen Maßnahmen erhöht in vielen Bereichen nachweislich die Befundqualität und die zeitliche Effizienz. Ziel der vorliegenden Studie ist es, die Befundqualität von Freitextbefunden (FTR) und SR im Hinblick auf Vollständigkeit, Effizienz und Benutzerzufriedenheit zu vergleichen. Patienten und Methoden: SR wurde bei schlafmedizinischen Patienten zur Erhebung von Anamnese, klinischer und technischer Untersuchungsbefunde sowie zur Erstellung korrespondierender Arztbriefe durch Assistenzärzte in fortgeschrittener Weiterbildung benutzt. Korrespondierende FTR und SR wurden anschließend bezüglich Befundqualität und Zeitaufwand ausgewertet. Ferner erfolgte eine Evaluation der Benutzerzufriedenheit mittels eines Fragenbogens mit visueller Analogskala (VAS).

Ergebnisse: Die SR zeigten eine signifikant höhere Befundvollständigkeit in allen Teilaspekten ( $92,8 \%$ vs. $52,6 \%, p<0,001)$. Die benötigte Zeit bei SR war dabei signifikant geringer als bei FTR (10,2 min vs. 16,8 min, $p<0,001$ ) und die Benutzerzufriedenheit signifikant höher (VAS 8,3 vs. $2,2, p<0,01)$

Schlussfolgerungen: SR in der somnologischen Sprechstunde liefert im klinischen Alltag eine überlegene Befundqualität, eine höhere zeitliche Effizienz und eine gesteigerte Benutzerzufriedenheit. Aufgrund dessen sollte SR in der schlafmedizinischen Befunderstellung empfohlen werden Schlüsselwörter: SBAS, Schlafapnoe, Digitalisierung, Strukturierte Befunderfassung, Diagnostik

\section{P 14}

Eine retrospektive Studie zur Etablierung des Lausanne NoSAS (Neck, Obesity, Snoring, Age, Sex) Score als Identifikator des obstruktiven Schlafapnoesyndrom

\section{*M. Krol, E. Cauza, A. Ecker}

Herz Jesu Krankenhaus Wien, Schlaflabor, Wien, Österreich

Fragestellung: Die vorliegende Arbeit beschäftigt sich mit der Frage, ob die beiden Screeningscores, der Epworth Sleepiness Scale und der NoSAS Score, Unterschiede hinsichtlich der Korrelation zwischen Score und der Diagnose eines obstruktiven Schlafapnoesyndroms aufweisen. Dazu wird der NoSAS Score dem ESS gegenübergestellt, um zu erkennen, welcher der beiden Screeningscores das Vorhandensein beziehungsweise das Fehlen des obstruktiven Schlafapnoesyndroms exakter zuordnet und somit als Identifikator besser geeignet ist. Aufgrund der geringen Anzahl an Schlaflaboratorien und der hohen Kosten, die mit einer Polysomnographie verbunden sind, soll derjenige Screeningscore etabliert werden, welcher möglichst wenige Patient ${ }^{\star}$ innen ohne OSAS einer Polysomnographie unterzieht, jedoch dennoch alle OSAS-Patient*innen detektiert. Demnach ist das Ziel die Erhöhung der Effizienz dieser Voruntersuchung.

Patienten und Methoden: Für die Studie wurden die Daten von Patient ${ }^{*}$ innen verwendet, welche im Jahr 2018 sowie im Jahr 2019 einer Polysomnographie im Schlaflabor des Herz-Jesu Krankenhauses in Wien unterzogen wurden. Eine Polysomnographie fand bei jenen Patient*innen statt, bei denen aufgrund eines positiven Screeningscore eine weitere Untersuchung erforderlich war beziehungsweise bei Patient ${ }^{*}$ innen, welche trotz negativen ESS-Score Beschwerden zeigten. Insgesamt haben 2317 Patient ${ }^{*}$ innen an der Studie teilgenommen, von denen 1097 aus dem Jahr 2018 und 1220 aus dem Jahr 2019 stammen. Mithilfe dieser Kontingenztabellen wurden für den ESS und den NoSAS Score der positiv-prädiktive Wert, der negativ-prädiktive Wert, die Sensitivität, die Spezifität sowie die Accuracy berechnet. Der wichtigste Parameter und somit die Hauptzielgröße für diese Arbeit ist die Accuracy. Im Anschluss an die deskriptivstatistische Auswertung erfolgt die Hypothesenprüfung mithilfe des McNemar-Test.

Ergebnisse: Für den Epworth Sleepiness Scale wurden eine Sensitivität von $36,5 \%$, eine Spezifität von $72,8 \%$, ein positiv-prädiktiver Wert von $94,3 \%$ und ein negativ-prädiktiver Wert von 8,5\% ermittelt. Die Accuracy des ESS beträgt 39,2\%. Für den NoSAS Score wurde eine Sensitivität von
$81,9 \%$, eine Spezifität von $67,6 \%$, ein positiv-prädiktiver Wert von $96,9 \%$, ein negativ-prädiktiver Wert von 23,2\% sowie eine Accuracy von 80,9\% berechnet. Durch die Gegenüberstellung der beiden Screeningscores ist ersichtlich, dass der NoSAS Score im Vergleich zum ESS eine höhere Sensitivität sowie höheren positiv-prädiktiven Wert aufweist.

Schlussfolgerungen: Die Studie ergab, dass der NoSAS Score im Gegensatz zum Epworth Sleepiness Scale eine höhere Sensitivität, einen höheren positiv-prädiktiven Wert, einen höheren negativ-prädiktiven Wert sowie eine deutlich höhere Accuracy aufweist. Durch die Hypothesenprüfung konnte die Alternativhypothese angenommen werden. Es besteht somit ein signifikanter Unterschied zwischen den Anteilen der richtig erfassten Testergebnisse beim Epworth Sleepiness Scale und dem NoSAS Score. Die Verwendung des NoSAS Scores in der Routine wäre somit ein effektives und einfach etablierendes Hilfsmittel zur Identifizierung von Patient*innen mit obstruktiven Schlafapnoesyndrom. In weiteren Studien könnte untersucht werden, welcher Cut-off-Wert des NoSAS Scores am geeignetsten ist und die Effizienz dieser Voruntersuchung weiter erhöht.

Schlüsselwörter: NoSAS, ESS, OSAS, Präambulanz, Accuracy

\section{P 15}

Eine retrospektive Studie zur Etablierung des ET-AHI

(Effectiveness of treatment Apnea Hypopnea Index) als Parameter für den CPAP (continuous positiv airway pressure) Therapieerfolg

*M. Krol, E. Cauza, J. Cicak

Herz Jesu Krankenhaus Wien, Schlaflabor, Wien, Österreich

Fragestellung: Da der gängige Complianceparameter in Prozent (=Therapietreue) nicht die effektive reale Veränderung des AHI zeigt und dieser prozentuelle Wert von der Nutzung der Therapie abhängt, ist es notwendig ein quantitatives Maß für die Einhaltung und gleichzeitig den Erfolg der CPAP-Therapie im Schlaflabor zu etablieren. Zusätzlich würde dieser Parameter sowohl die Einhaltung als auch den Erfolg der Therapie wiedergeben. Ziel der vorliegenden retrospektiven Studie ist die Etablierung des ETAHI als Faktor für die Messung des Erfolgs und gleichzeitig die Einhaltung der Therapie, für PatientInnen, welche eine CPAP-Therapie verwenden. Es soll ein mögliche Korrelation zwischen Compliance-Prozentwert und ET-AHI analysiert werden. Der ET-AHI soll im besten Fall als Parameter für die Kontrolle der Therapie in Schlaflaboratorien eingesetzt werden.

Patienten und Methoden: Die Kohorte wurde retrospektiv so ausgewählt, dass bei allen PatientInnen, innerhalb der Jahre 2018 und 2019, eine Kontrolle nach vier Monaten CPAP-Behandlung stattfand. Der Stichprobenumfang der vorliegenden Studie beinhaltet Datensätze von 1174 PatientInnen, 503 aus dem Jahr 2018 und 671 PatientInnen aus dem Jahr 2019. Für die Erhebung des ET-AHI wurden AHI-Werte von Polysomnografiensowie AHI- und Compliance-Werte während der Therapie durch die häusliche Überwachung (Auslesung der Speicherkarte), aus der klinischen Routineversorgung, verwendet und mittels folgender Formel berechnet: $\mathrm{ET}-\mathrm{AHI}=(\mathrm{AHI}$ mit Therapie $\mathrm{x} \%$ Compliance $)+($ Diagnose-AHI $\mathrm{x} \%$ Non-Compliance)

Da es sich bei den Datensätzen der vorliegenden Arbeit um keine Normalverteilung handelte, wurde die Korrelation zwischen Compliance Prozentwert und ET-AHI mittels Korrelationskoeffizient Spearman's rho statistisch berechnet und in einem Scatterplot dargestellt.

Ergebnisse: Über $50 \%$ aller PatientInnen zeigen bei Verwendung des Kontroll-AHIs einen Therapieerfolg. Jedoch spiegelt der Kontroll-AHI nur die Ereignisse während der Verwendung der CPAP-Therapie wieder. Hierbei muss eine tägliche Nutzung der Therapie von zumindest $4 \mathrm{~h}$ erfolgen. Der ET-AHI zeigt bei 288 PatientInnen, das sind rund $24 \%$ der Studienkohorte, einen Therapieerfolg an. Je kleiner der Compliance Prozentwert ist, desto mehr streuen die Werte des ET-AHI. Aufgrund des linearen Zusammenhangs konnte der Korrelationskoeffizient Spearman's rho zur Bewertung der Korrelation von ET-AHI und Compliance angewendet werden. Bei der vorliegenden Studie wurde ein Wert von $-0,82$ berechnet. Somit zeigt sich, dass zwischen ET-AHI und Compliance \% eine negativer Zusammenhang besteht: Je niedriger die Compliance \%, desto höher der ET-AHI. Weiters wurde 
berechnet, ob ein signifikanter Unterschied zwischen dem errechneten Korrelationskoeffizienten und 0 herrscht. Dies ergab einen $p$-Wert von $<2,2 \mathrm{e}-16$. Schlussfolgerungen: In dieser Studie wurde ersichtlich, dass es sich beim ET-AHI um einen Parameter handelt, der einen plausiblen Ist-Zustand der PatientInnen mit CPAP-Therapie wiedergibt. Der ET-AHI könnte nicht nur ein wichtiger Parameter für den CPAP-Therapieerfolg sein, sondern auch für andere Beatmungstherapien und alternative Behandlungen. $\mathrm{Zu}$ künftig könnten unterschiedliche Therapiearten anhand des ET-AHI miteinander vergleichen werden, um anschließend die für die PatientInnen effektivste Therapie zu wählen. In nachfolgenden Studien sollte eine längere Verwendung der Therapie angesetzt werden, um gegebenenfalls eine höhere Aussagekraft des Langzeitparameters zu zeigen.

Schlüsselwörter: Polysomnografie, ET-AHI, Compliance, CPAP Therapie, Überdrucktherapie

\section{P 16}

\section{Vergleich der Verteilung der Schlafpositionen bei zwei etablierten Verfahren zur ambulanten schlafmedizinischen Diagnostik}

*R. Birk, B. Sophia, C. E. Müller, H. Li, B. A. Stuck

Universitäts HNO-Klinik Marburg, HNO, Marburg, Deutschland

Fragestellung: Bei der obstruktive Schlafapnoe (OSA) sind die Atmungsstörungen häufig in der Rückenlage-verstärkt und manchmal sogar nur auf diese beschränkt. Zur Diagnostik der OSA wird in Deutschland initial i.d.R. eine Polygraphie durchgeführt. Eine weitere validierte diagnostische Möglichkeit ist die auf peripherer arterieller Tonometrie (PAT) basierende Untersuchung (WatchPAT ${ }^{\oplus}(\mathrm{WP})$ ). Unsere Hypothese ist, dass die ambulante Polygraphie durch die relativ umfangreichen Kabelverbindungen zu einer verstärkten Rückenlage führt. Dies kann insbesondere im Fall einer lagebezogenen Schlafapnoe systematisch überschätzte Schweregrad-Indices zur Folge haben. Postuliert wird ferner, dass der subjektive Schlafkomfort von Patienten, welche eine Polygraphie erhalten, im Gegensatz zur WatchPAT ${ }^{\oplus}$-Untersuchung schlechter und diese anfälliger für technische Ausfälle ist.

Patienten und Methoden: 43 Patienten erhielten in randomisierter sowohl eine Polygraphie als auch eine WatchPAT ${ }^{\oplus}$ Untersuchung an zwei aufeinanderfolgenden Nächten. Anschließend werden die schlafmedizinischen Daten ausgewertet und vergleichen. Die Patienten erhalten außerdem Fragebögen zum Schlafkomfort. Zusätzlich wurden Signalverluste und die Notwendigkeit der Wiederholung der Untersuchung dokumentiert.

Ergebnisse: Die Daten zeigen einen besseren subjektiven Schlafkomfort bei der WP-Untersuchung (86\%). Auch bevorzugen $95 \%$ der Patienten das WP im Gegensatz zur Polygraphie. Die WP Untersuchung musste insgesamt $3 \times$ wiederholt werden, die Polygraphie $5 \mathrm{x}$. Bezüglich der Lageverteilung zeigt sich eine Tendenz zu mehr Rückenlage bei der WP Untersuchung. Bisher wiesen 12 Patienten mehr Rückenlage mit der Polygraphie auf, während 23 Patienten mit dem WP mehr Rückenlage zeigten. Bei 8 Patienten könnte kein Unterschied nachgewiesen werden.

Schlussfolgerungen: Die Daten unserer Studie zeigen einen subjektiv besseren Schlafkomfort und eine Bevorzugung des WatchPAT ${ }^{\circledR}$ im Vergleich zur Polygraphie durch die Patienten. Unsere Hypothese, dass die ambulante Polygraphie zu einer verstärkten Rückenlage führt, könnte bisher nicht bestätigt werden. Es zeichnet sich eher eine gegenteilige Tendenz ab. Schlüsselwörter: OSA, Rückenlage, Schlafkomfort, WatchPAT, Polygraphie

\section{P 17}

Prävalenz, Inzidenz und Charakteristika der CPAP behandelten OSA-Patienten mit residualer EDS - Eine bevölkerungsrepräsentative Krankenkassendaten-Studie in Deutschland

${ }^{*}$ G. Nilius', U. Kallweit ${ }^{2}$, D. Trümper ${ }^{3},{ }^{*}$ T. Vogelmann ${ }^{4},{ }^{*}$ T. Schubert ${ }^{4}$ ${ }^{1} \mathrm{KEM}$ | Evang. Kliniken Essen-Mitte gGmbH, Pneumologie, Essen, Deutschland; ${ }^{2}$ Universität Witten/Herdecke, Institut für Immunologie, Witten, Deutschland; ${ }^{3}$ Jazz Pharmaceuticals Germany $\mathrm{GmbH}$, München, Deutschland; ${ }^{4}$ LinkCare GmbH, Stuttgart, Deutschland
Fragestellung: Residuale exzessive Tagesschläfrigkeit (R-EDS) ist ein Symptom bei Patienten mit obstruktiver Schlafapnoe (OSA). Bislang beruhten epidemiologische Schätzungen zu R-EDS bei OSA in Deutschland auf internationalen Studien. Das Ziel dieser Studie war daher (i) die Schätzung der alters- und geschlechtsspezifischen Prävalenz und Inzidenz und (ii) die Beschreibung der Charakteristika der OSA-Patienten mit residualer EDS unter CPAP-Therapie in Deutschland.

Patienten und Methoden: Diese Kohortenstudie nutzte die InGef-Forschungsdatenbank, einen anonymisierten repräsentativen Datensatz von 4 Mio. gesetzlich versicherten Personen in Deutschland. OSA-Patienten in CPAP-Therapie mit Anzeichen für eine residuale EDS, oder für eine EDS, die durch eine primäre OSA-Therapie nicht zufriedenstellend behandelt wurde, wurden anhand eines detaillierten Diagnostikschemas (u. a. Facharztbesuche, CPAP-Verordnung, erneute Polygraphie oder Polysomnographie und diagnostische Maßnahmen) zur Beantwortung der Fragestellung (i) im Jahr 2016 und zur Beantwortung der Fragestellung (ii) von 20142018 aufgegriffen. Eingeschlossen wurden 18 bis 80 Jahre alte Patienten. Ergebnisvariablen waren Prävalenz, Inzidenz und Patientencharakteristika sowie diagnostische Maßnahmen.

Ergebnisse: In der Datenbank wurden 1298 Patienten identifiziert, hochgerechnet auf Deutschland entspricht das 34.107 prävalenten OSA Patienten mit residualer EDS nach Einleitung einer CPAP-Therapie. 67,9\% der Patienten waren männlich. Das Durchschnittsalter lag bei 61 Jahren, $85 \%$ der Patienten waren $\geq 50$ Jahre alt. Die Jahresinzidenz lag in der Datenbank bei 203, hochgerechnet auf Deutschland bei 5327 Patienten.

Die häufigsten Komorbiditäten waren art. Hypertonie (77\%), Rückenschmerz (62\%) und depressive Episoden (57\%). $46 \%$ erhielten Antidepressiva und $45 \%$ waren in psychotherapeutischer Behandlung. Von den Beschäftigten waren im Jahr vor Diagnose $58 \%$ arbeitsunfähig mit einer mittleren Dauer von 71 Tagen. Die Patienten hatten im Jahr vor Diagnose 10 Facharztkonsultationen.

Schlussfolgerungen: Bei etwa $6,4 \%$ aller initial mit CPAP behandelten OSA-Patienten konnten Anhaltspunkte für eine residuale EDS gefunden werden. Die Hälfte dieser Patienten befand sich ich Psychotherapie oder wurde mit Antidepressiva behandelt, was auf eine hohe psychische Krankheitslast hinweisen könnte.

Schlüsselwörter: EDS, OSA, real-world evidence, Prävalenz, Abrechnungsdaten

\section{P 18}

\section{Sleep gets under your skin}

${ }^{*}$ C. Mann', P. Staubach', J. Roth', H. G. Weeß', J. Wegner'

'Universitätsmedizin, Hautklinik, Mainz, Germany; ${ }^{2}$ Pfalzklinikum, Schlafmedizin, Klingenmünster, Germany

Objectives: Erythropoietic protoporphyria is a rare dermatologic disease associated with acute phototoxicity and limited therapy options. Its far reaching impact on various aspects in life, including sleep, is yet to be fully unraveled.

Methods: In our validated-questionnaire-based study we asked 12 patients with Erythropoietic protoporphyria (EPP) to rate their insomnia (insomnia severity index; ISI), their daytime sleepiness (Epworth Sleepiness Scale, ESS), their quality of life (DLQI), their anxiety and depression (hospital anxiety and depression scale), and their general well-being (WHO questionnaire and general health questionnaire) before treatment with afamelanotide and at 3 consecutive visits during spring, summer and winter time. Furthermore, we asked them to name possible reasons for their sleeping problems.

Results: All of the patients reported pain and burning as a major problem during sleep. We saw a significant impact on quality of life, anxiety and depression, as well as an increase in the ISI score and in the ESS. We saw an improvement under therapy, but nonetheless season-dependent differences.

Conclusions: Sleep plays an important role even in rare dermatologic diseases and has a significant impact on quality of life. Adequate disease therapy is essential for healthy sleep.

Keywords: Sleep, Insomnia, Dermatology, Quality of life, Skin disease 


\section{P 19}

\section{Long-term sleep variability of patients with cystic fibrosis}

${ }^{*}$ C. M. Linker 1,2, S. Straßburg' ${ }^{2}$ S. Dietz-Terjung ${ }^{2}$, M. Welsner' ${ }^{2}$ S. Brato ${ }^{4}$, C. Taube ${ }^{2}$, C. Schöbel', J. Götze', G. Weinreich ${ }^{2}$

${ }^{1}$ Technische Universität Dortmund, Dortmund, Germany; ${ }^{2}$ Ruhrlandklinik Essen, Essen, Germany; ${ }^{3}$ SWG Sportwerk GmbH \& Co. KG, Dortmund, Germany

Objectives: Poor sleep in patients with cystic fibrosis (CF) is often observed but rarely over a long period due to cost-expensive polysomnography (PSG). Therefore, alternative technical approaches are welcome in order to analyze long-term sleep variability at home. To control measurement circumstances as much as possible, we first aimed to determine sleep variability of CF patients during their entire hospital stay.

Methods: Patients used a single-channel, minimal-contact device that measures motion by utilizing the piezo-electric effect (VitaLog SWG Sportwerk GmbH \& Co. KG, Dortmund, Germany). To analyze sleep variability the devices were used during the whole hospital stay.

Results: Forty-five CF patients (19 female, age $30.6 \pm 10.5$ y) were included. Patients" hospitalisation was initiated due to prophylactic antibiotics therapy or acute exacerbation. Altogether 298 nights has been analyzed. Total sleep time (TST) was $7.1 \pm 2.6 \mathrm{~h}$ on day 1 and $6.1 \pm 2.1 \mathrm{~h}$ at the last day $(p=0.03)$. Time in bed (TIB) was $9.6 \pm 3.1 \mathrm{~h}$ at the first day and $8.8 \pm 2.3 \mathrm{~h}$ at the last day $(p=0.07)$. There was a decrease in TST in 25 patients $(55.6 \%)$ and in TIB in $22(48.9 \%)$ during the hospital stay.

Conclusions: Significant, but only slight changes in sleep parameters can be found during hospital stay in CF patients. Longer measurement periods at home are required in order to gain profound knowledge on natural sleep behaviour and quality. This study showed that VitaLog is an appropriate tool for long-term examination of sleep.

Keywords: Cystic fibrosis, Sleep variability, Hospitalisation, Telemedicine, Minimal-contact device

\section{P 20}

Untersuchung von Schlafstoerungen als moeglicher praedisponierender Faktor fuer Anfaelle bei Patienten mit Neuromyelitis Optica

\section{*S. Rezaali, A. Naser Moghadasi, M. A. Sahraian}

MS Research Center, Sian Hospital, Teheran, Iran

Fragestellung: Neuromyelitis Optica (NMO oder Devic) ist eine chronische und neurologische Autoimmunerkrankung mit staendigen Rueckfaellen und Abklingen, deren Anfaelle zur Entkraeftung des Patienten und folglich zur Qualitaetsminderung seines Lebens fuehren koennen. Die taeglich Muedigkeit und der Mangel am geeigneten Schlaf in der Nacht kann den Rueckfall der Anfaelle in der Krankheit verursachen. Das Wissen und die Erkenntnis der Risikofaktoren, die zu den Anfaellen fuehren, kann eine wichtigen Beitrag leisten sowie eine wichtige Rolle spielen, um die entkraeftenden Anfaelle zu verhindern und die Lebensqualitaet der Betroffenen zu verbessern. Deswegen befasst man sich in diser Studie mit der Erforschung der Schlafstoerung als eine moegliche Ursache, die die Patienten anfaellig fuer die Anfaelle in dieser KrankheitNMO) macht Patienten und Methoden: In dieser Case-Controle Studie wurden $60 \mathrm{Neu}$ romyelitis Optica Erkrankten als Experimentalgruppe/Kontrollgruppe mit dem Kriterium 2015 erforscht, von denen 30 Personen in der Genesungsphase (Experimentalgruppe) und 30 Personen in der Anfallphase (zeugen/ Kontrollgruppe) waren. Die Schlafqualitaet im letzten Monat bei beiden Gruppen wurde nach dem Fragebogen PSQ1 erforscht und die Noten/Faelle mehr als 5 wurden als ungeeignete Schlafqualitaet mit einander verglichen. Alter, Geschlecht und Antikoerper-Situation (NMO) und MRIStatus vor dem Anfall, Begleitung der Autoimmunerkrankung, die Dauer der Infizierung und die frueheren Symptome der Patienten wurden erforscht und aufgezeichnet. Zudem wurde der Zusammenhang zwichen der Schlafqualitaet und jedem dieser Parameter geforscht

Ergebnisse: In der Experimentalgruppe hatten 16 Personen (53,3\%) und in der Kontrollgruppe 5 Personen (16,6\%) Schlafstoerungen. Das zeigt einen bedeutenden Unterschied zwischen beiden Gruppen $(P=0,001)$. Faktoren wie aelter sein, wieblich sein, wenn Antikoerper NMO positive Funde im MRI, die Begleitung der Autoimmunerkrankung, laenger Erkrankung und die frueheren Symptome der Patienten in der Experimentalgruppe hatten Auswikungen auf die Schlafstoerungen $(P<0,05)$ Schlussfolgerungen: Insgesamt wird aus allen Ergebnissen so schlussfolgert, dass die Schlafstoerung ein moeglicher Ausloeser ist, der die Patienten anfaellig fuer die Anfaelle in dieser Krankheit (Neuromyelitis Optica) macht. Aus diesem Grund kann der Verbesserung der Schlafqualitaet die Reduzierung der Rueckfaelle der Krankheitssymptome verursachen. Schlüsselwörter: Neuromyelitis Optica, Schlafstoerungen, Devic, NMORisikofaktor, NMO-Anfall

\section{P 21}

Die Ballistokardiographie misst die Herzfrequenz anhand der mechanischen Körperschwingungen aus der Herzbewegung. Ein neuartiges nicht-invasives Gerät unter der Matratze schätzt die Herzfrequenz durch Ballistokardiographie. Vier Algorithmen wurden entwickelt und in einer kleinen Studie getestet

${ }^{*}$ R. Seepold ${ }^{1,2}$, M. Conti ${ }^{3}$, N. Martinez Madrid ${ }^{2,4}$, C. Aironi ${ }^{3}$, S. Orcioni ${ }^{3}$, M. Gaiduk $k^{1,5}$

${ }^{1}$ HTWG Konstanz, Konstanz, Deutschland; ${ }^{2}$ I. M. Sechenov First Moscow State Medical University, Moskau, Russische Föderation; ${ }^{3}$ Università Politecnica delle Marche, Ancona, Italien; ${ }^{4}$ Reutlingen University, Reutlingen, Deutschland; ${ }^{5}$ University of Seville, Sevilla, Spanien

Fragestellung: Für die Überwachung des Schlafs zu Hause sind nichtinvasive Methoden besonders gut anwendbar. Die Signale, die häufig überwacht werden, sind Herzfrequenz und Atemfrequenz. Die Ballistokardiographie (BCG)ist eine Technik, bei der die Herzfrequenz aus den mechanischen Schwingungen des Körpers bei jedem Herzzyklus gemessen wird. Kürzlich wurden Übersichtsarbeiten veröffentlicht [1,2]. Die Untersuchung soll in einem ersten Ansatz bewerten, ob die Herzfrequenz an-

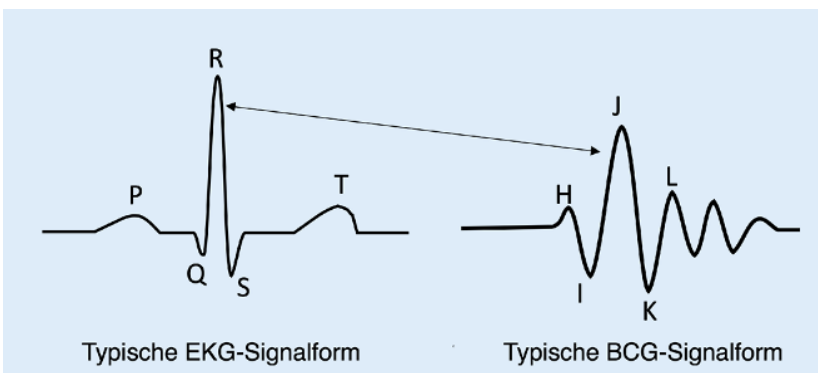

Abb. 1 | P 21 ॥ Typische Form des EKG- und BCG-Signals

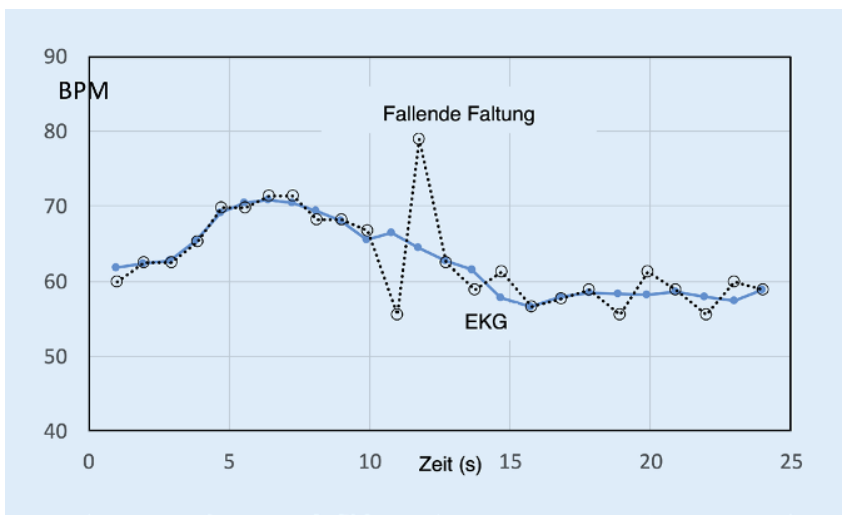

Abb. 2 | P $21 \Delta$ BPM mit fallender Faltung und EKG-Signal 
hand von BCG erkannt werden kann. Die wesentlichen Randbedingungen sind, ob dies gelingt, wenn der Sensor unter der Matratze positioniert wird und kostengünstige Sensoren zum Einsatz kommen.

Fragestellung: Es wurde ein BCG-System entwickelt und in einer kleinen Studie mit 21 Patienten in je 20-minütigen Sitzungen getestet. Als Referenz diente ein 12-Kanal EKG. Die Abtastfrequenz der EKG- und BCG-Signale betrug $1 \mathrm{kHz}$ bzw. $50 \mathrm{~Hz}$. Es wurden mehrere Beschleunigungssensoren evaluiert und eine Hardware für die Datenerfassung und -speicherung entwickelt. Die Signalverarbeitung für die Berechnung der Herzfrequenz aus dem Beschleunigungssignal wurde in MATLAB entwickelt und dann in Python kodiert. Der QRS-Komplex im EKG-Signal entspricht dem IJKSpitzenwert im BCG-Signal (s. • Abb. 1). Das BCG-Signal wurde in einem Zeitfenster von $10 \mathrm{~s}$ durch zwei Referenzmuster (steigend und fallend) normalisiert. Er wurden vier Algorithmen verwendet, um die Periode des Signals und damit die Herzfrequenz in Schläge pro Minute (BPM) zu schätzen. Die Algorithmen basieren auf Autokorrelation oder Faltung mit dem Referenzsignal.

Ergebnisse: Es wurden drei Beschleunigungssensoren untersucht: MPU6050 von InvenSense für $2 €$, ADXL355Z von Analog Devices für $40 €$ und PCB 353-B43 von PCB Piezotronics als Referenz für $1000 €$. Ausgewählt wurde ADXL355Z, der das beste Preis-Leistungs-Verhältnis bot. Alle entwickelten Algorithmen liefern akzeptable Ergebnisse im Vergleich zum Referenz-EKG-Signal. Die Ergebnisse mit steigender Korrelation sind am schlechtesten, da in einigen Fällen ein falscher Peak erkannt wird. Die drei anderen Algorithmen liefern vergleichbare Ergebnisse. Beispielsweise wurde bei einer $25 \mathrm{~s}$ Messung und einem EKGBPM von 62,8 folgendes berechnet: 63,0 (fallende Faltung), 63,2 (fallende Korrelation) und 63,3 (steigende Faltung). - Abb. 2 zeigt den BPM mit fallender Faltung und dem EKGSignal.

Schlussfolgerungen: Das entwickelte System ermöglicht die Schätzung der Herzfrequenz mit einem kostengünstigen, nicht-invasiven Gerät, das unter eine gewöhnliche Matratze eines Standardbetts gelegt wird. Die Abschätzung der BPM ist aufgrund der geringen Amplitude des unter der Matratze gemessenen BCG-Signals im Vergleich zum Rauschen anspruchsvoll. Die erzielten Ergebnisse hängen stark von der Auswahl des Beschleunigungssensors, der mechanischen Struktur, die den Sensor trägt und der Position unter der Matratze ab. Vorläufige Ergebnisse zeigen eine gute Übereinstimmung der BPM-Schätzung mit EKGs. Die vollständige Analyse ist noch nicht abgeschlossen. Er laufen weitere Experimente, um statistisch relevante Ergebnisse zu erhalten und den Verarbeitungsalgorithmus zu verbessern.

\section{Literatur}

1. Sadek I, Biswas J (2019) Nonintrusive heart rate measurement using ballistocardiogram signals: a comparative study. SIViP 13(3):475-482

2. Conti M et al (2018) A Review of Health Monitoring Systems Using Sensors on Bed or Cushion. LNCS 10814:347-358

\section{P 22}

\section{Adhärenzverhalten bei kognitiver Verhaltenstherapie für Insomnie: Ergebnisse aus einer randomisierten kontrollierten klinischen Studie}

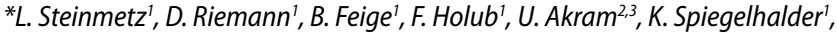
A. F. Johann ${ }^{1,4}$

'Klinik für Psychiatrie und Psychotherapie, Universitätsklinik Freiburg, Medizinische Fakultät, Albert-Ludwigs-Universität Freiburg, Freiburg, Deutschland; ${ }^{2}$ Sheffield Hallam University, Department of Psychology, Sociology and Politics, Sheffield, Vereinigtes Königreich; ${ }^{3}$ University of Oxford, Sleep and Circadian Neuroscience Institute (SCNi), Nuffield Department of Clinical Neurosciences, Oxford, Vereinigtes Königreich; ${ }^{4}$ Albert-Ludwigs-Universität Freiburg, Medizinische Psychologie und Medizinische Soziologie, Freiburg, Deutschland

Fragestellung: Insomnie ist eine häufig schwerwiegende und chronische Erkrankung, die erhebliche Auswirkungen auf die Lebensqualität hat. Die in klinischen Leitlinien empfohlene Erstbehandlung ist die kognitive Ver- haltenstherapie für Insomnie (KVT-I). Frühere Studien zeigen, dass bei chronischen Erkrankungen und insbesondere bei Schlafstörungen die Einhaltung der Behandlungsempfehlungen (Adhärenz) im Vergleich zu akuten Erkrankungen gering ist. Studien zur KVT-I untersuchen jedoch selten die Adhärenz, oder die Bewertung dieser ist auf die durchschnittliche Anzahl der besuchten Therapiesitzungen begrenzt. Ziel dieser Studie ist es, den Zusammenhang zwischen einem indirekten Maß für Adhärenz bei KVT-I und der Wirksamkeit der Behandlung zu untersuchen. Die Daten stammen aus einer randomisierten kontrollierten Studie, in der die Auswirkung von KVT-I auf frühe Marker für Herz-Kreislauf-Erkrankungen untersucht wurde.

Patienten und Methoden: Die Stichprobe bestand aus 46 Patienten (je 23 Patienten in der Interventionsgruppe und der Kontrollgruppe), die die DSM-5-Kriterien für Insomnie erfüllten mit KVT-I behandelt wurden. Für eine Schätzung der Adhärenz bei der Schlafrestriktion wurde der auf dem Schlaftagebuch basierende Unterschied in der Bettzeit vor und nach der Behandlung berechnet und mit dem Unterschied in der Insomnieschwere vor und nach der Behandlung korreliert. Für die Präsentation bei dem Kongress ist eine detaillierte Analyse der Adhärenz verschiedener Komponenten von KVT-I anhand von Schlaftagebuchdaten und Videoaufnahmen der Behandlung geplant

Ergebnisse: Die ersten Ergebnisse zeigten, dass die Beteilung an der Therapie hoch war. Im Durchschnitt nahmen die Patienten an 7,3 $\pm 1,3$ Sitzungen von 8 Sitzungen in $8,9 \pm 3,0$ Wochen teil. Die Korrelation zwischen dem Unterschied in der Bettzeit vor und nach der Behandlung und dem Unterschied zwischen Schweregrad der Schlaflosigkeit vor und nach der Behandlung war für die gesamte Gruppe an Studienteilnehmern signifikant $(\mathrm{r}=0,42, p<0,01)$. Die Signifikanz der Korrelation wurde hauptsächlich durch eine Korrelation innerhalb der Kontrollgruppe bedingt $(\mathrm{r}=0,44$, $p=0,04)$. Die Korrelation innerhalb der KVT-I-Gruppe war nicht signifikant $(\mathrm{r}=0,19, p=0,39)$.

Schlussfolgerungen: KVT-I-Behandlungseffekte scheinen mit Veränderungen der Bettzeiten vor und nach der Behandlung in Zusammenhang zu stehen. Es bleibt unklar, ob das gewählte Maß ein guter Indikator für die Adhärenz ist, da

Schlüsselwörter: Insomnie, Adhärenz, KVTI, Schlafrestriktion, Sekundäre Analyse

P 23

\section{Wirkt SMR-Neurofeedback-Training bei Insomnie (doch)?}

*E. Liesa, T. Llera-Perez, R. Thees, C. Lange, A. Rupprecht, N. Tscharaktschiew

TU Chemnitz, Institut für Psychologie - Allgemeine \& Biopsychologie, Chemnitz, Deutschland

Fragestellung: Erhöhte körperliche und/oder psychische Anspannung (Hyperarousal) kann Insomnien begünstigen. Zur Behandlung wird u. a. Neurofeedbacktraining (NFB-Training) eingesetzt. Hierbei wird häufig das Feedback des Sensomotorischen Rhythmus (SMR) verwendet. Jedoch zeigte eine Placebo-kontrollierte Doppelblindstudie keine stärkeren Verbesserungen der Schlafqualität einer SMR-NFB-Gruppe im Vergleich zu einer Placebogruppe mit Schein-NFB. In einer ebenfalls doppelt blinden Placebo-kontrollierten Pilotstudie untersuchten wir, ob die Integration eines Ruhebildes (Video) in das NFB-Training subjektive Schlafqualität, Focussing und Grübeln verbessert und den SMR-Frequenzanteil erhöht. Als Placebo-NFB nutzten wir Biofeedback-Training (BFB-Training) des Hautleitwertes (Skin Conductance Level, SCL). Den Probanden wurden EEGund SCL-Elektroden angelegt, sodass die jeweilige Bedingung weder für die Probanden noch für die Versuchsleiter ersichtlich war.

Patienten und Methoden: Es nahmen 4 Probanden am Training teil, wobei $n=2$ Probanden das SMR-NFB-Training und 2 Probanden das SCL-BFBTraining absolvierten. Weitere 4 Probanden gehörten einer KG an, die kein Training erhielt. Ruhebilder werden als kognitiv-verhaltenstherapeutische Methode u. a. zur Behandlung von Insomnie eingesetzt. Im Rahmen des NFB-Trainings dient ein Ruhebild-Video dazu, (1) während des Trainings durch operante Konditionierung die gewünschte EEG-Aktivität zu belohnen (z. B. wenn das Video bei Erreichen der gewünschten EEG-Aktivität 
zum Laufen gebracht wird) sowie (2) durch die gedankliche Vorstellung des Videos beim Einschlafen oder bei nächtlichem Erwachen durch klassisches Konditionieren die gewünschte Hirnaktivität (hier: SMR) zu reaktivieren und so Hyperarousal zu reduzieren. Das Training erstreckte sich jeweils über 12 Sitzungen à 45 min.

Ergebnisse: Die grafische Auswertung der Pilotstudie gibt erste Hinweise darauf, dass SMR-NFB-Training mit Ruhebild zur Verbesserung der Schlafqualität, zur Verringerung des Grübelns und zur Verstärkung des SMR, jedoch nicht zur Reduzierung des Focussings beiträgt. Kruskal-Wallis- und Mann-Whitney-U-Tests ergaben Effektgrößen von $\eta^{2} 0,05$ bis $\eta^{2}$ 0,43 , waren jedoch aufgrund der sehr kleinen Stichprobe nicht signifikant. Schlussfolgerungen:

Die bisherigen Ergebnisse sollten mit größeren Stichproben genauer untersucht werden. Zudem sollten zur Beurteilung der objektiven Schlafqualität polysomnografische Analysen mit einbezogen werden.

Schlüsselwörter: Neurofeedback, Insomnie, SMR, Ruhebild, Biofeedback

\section{P 24 \\ Bedeutung und Behandlung von Alpträumen bei der Posttraumatischen Belastungsstörung \\ ${ }^{*}$ A. Deen}

Universitätsklinikum Hamburg-Eppendorf, Psychosen - Integrierte Versorgung, Hamburg, Deutschland

Fragestellung: Intensive und sehr belastende Alpträume sind ein häufiges Symptom der posttraumatischen Belastungsstörung (PTBS). Sie begünstigen komorbiden Substanzmissbrauch und Suizidalität (Raskind et al., 2007). Zugelassen für die Behandlung der PTBS sind nur die selektiven Serotonin-Wiederaufnahmehemmer (SSRI) Sertralin und Paroxetin $(\mathrm{Cu}-$ kor, Olden, Lee, Difede, 2010). Bisher konnte keine Evidenz für die Wirkung von SSRI bei Alpträumen nachgewiesen werden (Brownlow, Harb Ross, 2015). Viele PTBS-PatientInnen nutzen Cannabis aufgrund seiner Entspannung und Schlaf fördernden sowie angstlindernden Wirkung als Selbstmedikation (Betthauser, Pilz, Vollmer, 2015; Bonn-Miller, Vujanovic, Feldner, Bernstein, Zvolensky, 2007; Bremner, Southwick, Darnell, Charney, 1996). Klinische Daten geben Hinweise darauf, dass Cannabinoidagonisten wie Dronabinol zur Reduktion von Alpträumen und anderen PTBS-Symptomen führen können (Wilkinson, Radhakrishnan, D'Souza, 2016).

Schlussfolgerungen: Der Beitrag ordnet zunächst das Symptom der Alpträume bei der PTBS mit Daten aus einer eigenen longitudinalen Studie zu den akuten Folgen der Traumatisierung ein. Anschließend wird ein Überblick über Behandlungsmöglichkeiten der medikamentösen Alptraumtherapie gegeben. Darüber hinaus wird eine anlaufende randomisiert-kontrollierte Studie zur Behandlung von Alpträumen bei PTBS mit Dronabinol vorgestellt.

\section{P 25}

\section{Überlebensraten lungentransplantierter Patienten mit und ohne schlafbezogene Atmungsstörungen}

*J. Thyssen', C. Taube', V. Besa', C. Aigner'², M. Kamler' , U. Sommerwerck ${ }^{4}$ B. Kleibrink', G. Weinreich', C. Schöbe/ ${ }^{5}$

'Ruhrlandklinik, Universitätsmedizin Essen, Universität Duisburg-Essen, Pneumologie, Essen, Deutschland; ${ }^{2}$ Ruhrlandklinik, Universitätsmedizin Essen, Thoraxchirurgie und thorakale Endoskopie, Essen, Deutschland; ${ }^{3}$ Herzchirurgie Huttrop, Universitätsmedizin Essen, Essen, Deutschland; ${ }^{4}$ Krankenhaus der Augustinerinnen, Pneumologie, Köln, Deutschland; ${ }^{5}$ Ruhrlandklinik, Universitätsmedizin Essen, Schlafmedizinisches Zentrum, Essen, Deutschland

Fragestellung: Lungentransplantation (LTX) ist eine Schlüsselmethode zur Behandlung von Lungenerkrankungen im Endstadium. LTX-Patienten weisen einige Komorbiditäten auf, die mit schlafbezogenen Atmungsstörungen (SBAS) assoziiert sind. Es ist bekannt, dass die Prävalenz von SBAS bei lungentransplantierten Patienten hoch ist.
Ziel unserer Studie war, die Überlebensraten von LTX-Patienten mit und ohne SBAS zu ermitteln, da diese Fragestellung bislang noch nicht untersucht wurde.

Patienten und Methoden: Zwischen 2011 und 2014 schlossen wir 74 lungentransplantierte Patienten (44 Männer, Alter 53,7 $\pm 9,9$ Jahre, BMI $24,7 \pm 4,4 \mathrm{~kg} / \mathrm{m}^{2}$, Zeitspanne seit LTX 1 Monat bis zu 11 Jahren) in eine prospektive Studie ein. Indikationen für die Lungentransplantationen waren chronisch obstruktive Lungenerkrankung $(n=28)$, Idiopathische Lungenfibrose $(n=14)$, Cystische Fibrose $(n=8)$ und andere $(n=24)$. Um die Schlafcharakteristika zu untersuchen, wurden Polysomnographien durchgeführt. Es stellte sich eine hohe Prävalenz schlafbezogener Atmungsstörungen heraus (48 \%). Ein Follow-up der Studienpopulation fand bis 2019 statt. Mittels Kaplan-Meier-Analysen ermittelten wir die Überlebensraten lungentransplantierter Patienten mit und ohne SBAS.

Ergebnisse: Bei Verwendung eines Apnoe-Hypopnoe-Indexes (AHI) $\geq 5 / h$ als Cut-off-Wert zur Definition schlafbezogener Atmungsstörungen starben 8 von 21 Patienten mit SBAS und 18 von 53 ohne SBAS (33,9\% vs. $38 \%, p=0,43)$. Bei einem AHI $\geq 15 / h$ als Cut-off-Wert zur Ermittlung von SBAS verstarben 17 von 47 Patienten mit und 9 von 27 ohne SBAS $(33,3 \%$ vs. $36,1 \%, p=0,64)$.

Schlussfolgerungen: In unserer Kohorte beobachteten wir keinen statistisch signifikanten Unterschied zwischen den Überlebensraten lungentransplantierter Patienten mit und ohne SBAS.

Schlüsselwörter: Überleben, Lungentransplantation, SBAS, Apnoe-Hypopnoe-Index, Kaplan-Meier

\section{P 26}

\section{Patientenpräferenzen in der Behandlung der Obstruktiven} Schlafapnoe - Systematischer Review und Qualitative Analyse

*M. Braun, S. Dietz-Terjung, G. Weinreich, C. Taube, C. Schöbel Ruhrlandklinik Essen, Schlafmedizinisches Zentrum, Essen, Deutschland

Fragestellung: In vielen Gesundheitssystemen hat sich heute die Beteiligung der Betroffenen an Versorgungsentscheidungen, insbesondere bei chronischen Erkrankungen wie der OSA, durchgesetzt. Eine Voraussetzung für die Patientenbeteiligung ist die Kenntnis von Präferenzen zu Attributen von Behandlungsoptionen. Ziel der Studie war die Auswertung des aktuellen Forschungsstandes zu Patientenpräferenzen bei der OSABehandlung.

Patienten und Methoden: Zur Identifizierung relevanter Literatur wurden a priori Eignungskriterien definiert und Suchanfragen in MEDLINE und Google Scholar durchgeführt. Insgesamt wurden für den Zeitraum 01/2000-05/2020 zehn Arbeiten identifiziert, die zwischen 22 und 444 Patienten untersuchten. Die häufigste verwendete Methodik war (semi-) strukturierte Befragung $(n=5)$, gefolgt von Discrete Choice Experiment $(n=4)$ bzw. Fokusgruppeninterview $(n=1)$. Häufigstes Studienziel war die Ermittlung von Behandlungspräferenzen, wobei lediglich eine Studie tatsächlich ausgeübte Präferenzen untersuchte (Revealed preferences). Alle anderen ermittelten ausgedrückte Präferenzen (Stated preferences).

Ergebnisse: Trotz der hohen Prävalenz der OSA konnten nur wenige Arbeiten identifiziert werden, die zudem hinsichtlich Methodik eine gewisse Heterogenität aufweisen. Bislang durchgeführten Studien sind in ihrer Aussagekraft aufgrund niedriger Fallzahlen teilweise eingeschränkt. Untersuchungen aus Deutschland liegen bislang nicht vor. Discrete Choice Experimente (DCE), die als Goldstandard in der Ermittlung von Patientenpräferenzen gelten, wurden nur teilweise eingesetzt und untersuchten eher kleine Populationen. In den bisher durchgeführten DCE wurden langfristiger Therapieeffekt, Nebenwirkungen, kurzfristiger Nutzen und negativer Einfluss auf Lebensqualität als relevante Attribute von Behandlungen identifiziert, die den projizierten Nutzen beeinflussten.

Schlussfolgerungen: $\mathrm{Zu}$ Patientenpräferenzen bei Betroffenen mit OSA ist bislang nur wenig bekannt. Aus bislang veröffentlichten Arbeiten sind erste Nutzenwerte von Behandlungsoptionen erkennbar. Diese sind allerdings aufgrund spezifischer Gesundheitssystemrelevanter Unterschiede nicht bzw. nur eingeschränkt auf Deutschland übertragbar. Zudem sind bislang keine Untersuchungen vorhanden, die alle verfügbaren Therapieverfahren 
einschließen und so Rückschlüsse auf die Entscheidungsmöglichkeiten in der Versorgungssituation im Jahr 2020 zulassen. Dementsprechend besteht Bedarf an weiteren Untersuchungen zu dieser Fragestellung. Schlüsselwörter: Obstruktive Schlafapnoe, Präferenzen, Behandlung, Nutzenwertstudien, Discrete Choice Experimente

\section{P 27 \\ Efficacy of Pitolisant $20 \mathrm{mg}$ in reducing excessive daytime sleepiness for patients with obstructive sleep apnea syndrome: an individual patient data meta analysis}

${ }^{*}$ C. Caussé', P. Lehert ${ }^{2,3}$

${ }^{1}$ Bioprojet, Medical, Paris, France; ${ }^{2}$ University of Louvain, Faculty of Economics, Mons, Belgium; ${ }^{3}$ University of Melbourne, Faculty of Medicine, Melbourne, Australia

Objectives: Pitolisant is an Histamin H3-receptor antagonist/inverse agonist waking agent for the treatment of Excessive Daytime Sleepines (EDS) in adult narcolepsy patient. We synthesized evidence on its efficacy and safety at a dose of $20 \mathrm{mg}$ compared with placebo through an Individual patient data meta-analysis based on the two studies HAROSA-1 and -2 that have included Obstructive Sleep Apnea (OSA) patients presenting EDS and treated respectively with and without Continuous Positive Airway Pressure (CPAP) therapy.

Methods: Epworth Sleep Scale (ESS) and Oxford Sleep Resistance (Osler) tests were co-primary endpoints tested at 0.025 significance and Fatigue (Pichot Scale) was secondary. Intent to treat was our selection basis. For sensitivity purpose, three competing meta-analytical and three alternative missing data imputation methods were compared.

Results: A significant mean ESS reduction of -2.66 ([95\%CI -3.58, - 1.53], $p<0.001)$ was found with Pitolisant versus placebo and $79 \%$ more patients decreased final ESS to less than $10(\mathrm{RR}=1.79$ [95\%CI 1.35, 2.38], $p<0.001)$. The Osler Final/Baseline was also $16 \%$ better (ratio $=1.16$, [95\%CI 1.02, 1.32], $p=0.018$ ). A clinically meaningful EDS effect of Pitolisant measured by the aggregate Z-score on ESS and Osler was $0.59([0.36,0.82], p<0.001)$. Finally, a significant mean Pichot Fatigue reduction of -1.231 ([-2.285, $0.177], p=0.022$ ) was found. These effects were shown invariant across various subgroups of the population (age, gender, and work conditions). Finally these effects were not impacted as to whether or not CPAP was used. Conclusions: These results provide evidence of significant pitolisant efficacy on EDS and Fatigue symptoms in sleepy OSA patients versus placebo, evaluated by the ESS, Osler, EDS Z-score and Pichot Fatigue, irrespective of CPAP use.

Disclosure: Bioprojet sponsored this analysis. C. Caussé is an employee of bioprojet pharma. P. Lehert received honoraria from bioprojet.

Keywords: Pitolisant, Obstructive sleep apnea, Excessive daytime sleepiness, Meta analysis, Epworth Sleep Scale

\section{P 28}

Effects of solriamfetol on driving performance in participants with excessive daytime sleepiness associated with obstructive sleep apnoea

${ }^{*}$ F. Vinckenbosch', J. Asin'², N. de Vries ${ }^{3}$, P. Vonk ${ }^{3}$, C. Donjacour', G. J. Lammers 4,5 , S. Overeem ${ }^{6,7}$, H. Janssen ${ }^{6}$, G. Wang ${ }^{8}$, D. Chen ${ }^{8}$, L. Carter ${ }^{8}$, K. Zhou ${ }^{8}$, A. Vermeeren', J. Ramaekers'

${ }^{1}$ Maastricht University, Maastricht, Netherlands; ${ }^{2}$ Center for sleep medicine Amphia, Breda, Netherlands; ${ }^{3}$ OLVG, Amsterdam, Netherlands; ${ }^{4}$ Sleep-wake center SEIN, Leiden, Netherlands; ${ }^{5}$ Leiden University Medical Center, Leiden, Netherlands; ${ }^{6}$ Kempenhaeghe, Heeze, Netherlands; ${ }^{7}$ Eindhoven University of Technology, Eindhoven, Netherlands; ${ }^{8}$ Jazz Pharmaceuticals, Palo Alto, United States

Objectives: Excessive daytime sleepiness (EDS) in patients with obstructive sleep apnoea (OSA) is associated with an increased risk of driving accidents. Solriamfetol, a dopamine/norepinephrine reuptake inhibitor, is approved in the EU and US to improve wakefulness and reduce EDS in adults with OSA who use or have attempted to use therapy to treat the underlying airway obstruction (approved dose range, 37.5-150 mg/day). This study evaluated the effects of solriamfetol on on-road driving performance in participants with EDS associated with OSA.

Methods: In each period of this randomised, double-blind, placebo-controlled, crossover study (NCT02806895/EudraCT 2015-003930-28), driving performance during an on-road driving test was assessed at $2 \mathrm{~h}$ and $6 \mathrm{~h}$ post-dose following 7 days of treatment with solriamfetol $(150 \mathrm{mg} /$ day $\times 3$ days, then $300 \mathrm{mg} /$ day $\times 4$ days) or placebo. The primary endpoint-standard deviation of lateral position (SDLP), a measure of "weaving" (departure from a steady lane position), at $2 \mathrm{~h}$ post-dose-was compared between solriamfetol and placebo using a repeated mixed-effects analysis of variance model.

Results: Baseline characteristics reflected the broader OSA population $(\mathrm{N}=34 ; 88 \%$ male; mean age, 52 years; mean Epworth Sleepiness Scale score, 14.4; Maintenance of Wakefulness Test sleep latency, $14.3 \mathrm{~min}$ ). At $2 \mathrm{~h}$ post-dose, SDLP was significantly lower with solriamfetol (least squares [LS] mean [standard error; SE], $18.8 \mathrm{~cm}$ [0.63]) versus placebo (19.9 cm [0.63]): LS mean difference, $-1.1 \mathrm{~cm} ; 95 \%$ confidence interval (CI), $-1.9,-0.32 ; P=0.006$, indicating better performance with solriamfetol; 1 participant receiving solriamfetol and 4 receiving placebo failed to complete the driving test. At $6 \mathrm{~h}$ post-dose, SDLP with solriamfetol (LS mean [SE], $19.2 \mathrm{~cm}$ [0.63]) was significantly lower versus placebo $(20.0 \mathrm{~cm}$ [0.63]): LS mean difference, $-0.8 \mathrm{~cm} ; 95 \% \mathrm{CI},-1.58,-0.03 ; P=0.043 ; 3$ participants receiving solriamfetol and 7 receiving placebo failed to complete the driving test. Common adverse events $(\geq 5 \%)$ with solriamfetol were headache, nausea, insomnia, dizziness, and agitation.

Conclusions: Solriamfetol (300 mg/day) improved SDLP, an important measure of driving performance, at 2 and $6 \mathrm{~h}$ in participants with EDS associated with OSA.

Keywords: JZP-110, Sleep, Wake disorders, Sleep apnoea syndromes, Accidents, Automobiles

\section{P 29}

\section{Comparison of Modafinil and Pitolisant in Narcolepsy: a non inferiority meta-analytical approach}

\section{${ }^{*}$ C. Caussé', P. Lehert ${ }^{2,3}$}

${ }^{1}$ Bioprojet, Medical, Paris, France; ${ }^{2}$ University of Louvain, Faculty of Economics, Mons, Belgium; ${ }^{3}$ University of Melbourne, Faculty of Medicine, Melbourne, Australia

Ojectives: Pitolisant $(\mathrm{P})$ is a drug used in narcolepsy for Excessive Daytime Sleepiness (EDS) and cataplexy. We assessed the non-inferiority of Pitolisant efficacy compared with Modafinil (M).

Methods: Network meta-analysis (NMA) compared efficacy and safety of multiple treatments and multi-arm studies. Epworth Sleepiness Scale (ESS), Maintenance of Wakefulness Test (MWT), the Weekly Rate of Cataplexy (WRC) and safety constituted the main endpoints, EDS and Narcolepsy Z-scores and Benefit/Risk ratios considered as supportive measures. Pre-determined non inferiority margins of 1 score, and $1 \mathrm{~min}$ was fixed for the comparison of ESS and MWT, respectively.

Results: Among 312 articles, 10 Randomised Clinical Trials were assessed and eligible for NMA. For ESS, a non-significantly superior beneficial decrease of $-0.69([-2.18,0.79])$ in $\mathrm{P}$ arm provided evidence of non-inferiority compared with a Non Inferiority Margin $(\mathrm{NIM})=1(p=0.015)$. A MWT beneficial increase of $2.12 \mathrm{~min}([-0.95,5.19], p=0.18)$ in $\mathrm{P}$ arm compared to placebo, showed non-inferiority compared with NIM=-1. For cataplexy, the pitolisant effect was $-0.49([-0.86,-0.12]$, decrease of $\mathrm{WRC}=-4.3, p=0.012$ ), significantly superior to $\mathrm{M}$ in addition of non-inferiority. The Risk Ratio (RR) of Treatment suspected adverse events P/M was $\mathrm{RR}=0.86[0.44,1.24]$ in favor of $\mathrm{P}$ confirming non inferiority considering a safety margin of $\mathrm{RR}=1.25$ (tolerance of $25 \%$ ). For Narcolepsy Typel patients, a significant benefit was found in favor of $\mathrm{P}$ of 0.49 ([0.08, 1.03 ], $p=0.02)$. For Narcolepsy Type 2 patients, the observed benefit $P$ was $0.24([-0.19,0.70])$ considered as non-inferior based on $\mathrm{NIM}=0.2$. 
Conclusions: Modafinil produced a significant improvement in EDS (ESS and MWT), and an acceptable safety profile, without significant effect on cataplexy. Pitolisant is shown as non-inferior than modafinil in relieving EDS, and superior to modafinil in reducing the occurrence of cataplexies, thus pitolisant outranks modafinil when prescribed to Narcolepsy type 1 patients. In spite of a slight superiority of pitolisant in EDS relief, the two drugs can be considered as equally performing for the treatment of Narcolepsy type 2 patients.

Disclosure: Bioprojet sponsored this analysis. C. Caussé is an employee of bioprojet pharma. P. Lehert received honoraria from bioprojet.

Keywords: Narcolepsy, Non inferiority meta-analytica, Pitolisant, Modafinil, Excessive Daytime Sleepiness

\section{P 30}

\section{Solriamfetol zur Behandlung der Tagesschläfrigkeit bei Narkolepsie - Erste praktische Erfahrungen aus einem Narkolepsie-Zentrum}

${ }^{*}$ C. U. Mevers, C. Vetten, L. Lieberich, A. Triller, U. Kallweit Universtität Witten/Herdecke, Zentrum für Narkolepsie/Hypersomnien und Klin. Schlaf- und Neuroimmunologie, Witten, Deutschland

Fragestellung: Narkolepsie ist eine chronische neurologische Erkrankung, deren Hauptsymptome exzessive Tagesschläfrigkeit und Kataplexien sind. Die Behandlung beinhaltet Verhaltensmaßnahmen und eine symptomatische Pharmakotherapie, vor allem der Hauptsymptome. Solriamfetol ist ein Noradrenalin- und Dopamin-Wiederaufnahmehemmer und wurde Anfang 2020 von der EMA zur Behandlung der exzessiven Tagesschläfrigkeit bei Narkolepsie und obstruktiver Schlafapnoe zugelassen.

Seit Mitte Mai 2020 kann Solriamfetol zur Behandlung der Narkolepsie verordnet werden.

Ziel dieser prospektiven Beobachtungsstudie war die Beurteilung der Wirksamkeit, möglicher Nebenwirkungen, bestehender Ko-Medikation und Ko-Morbiditäten bei Patienten mit Narkolepsie im praktischen Alltag. Patienten und Methoden: Prospektiver Einschluss konsekutiver erwachsener Patienten mit Narkolepsie (Typ 1 und 2). Es wurden Patienten rekrutiert, die im Zentrum für Narkolepsie/Hypersomnien, Universität Witten/ Herdecke behandelt werden.

Erfassung der demographischen Daten, der klinischen Charakteristika der Narkolepsie; Ko-Morbiditäten und der Medikation vor und nach Umstellung. Auch wurde die Dosierung von Solriamfetol dokumentiert. Zur Beurteilung der Tagesschläfrigkeit wurde zudem der Fragebogen Epworth Sleepiness Scale (ESS) eingesetzt. Erfassung sämtlicher o. g. Daten vor Beginn der Therapie mit Solriamfetol und 3 Monate später.

Ergebnisse: Vorläufige Resultate werden präsentiert. Bis 09.06.2020 wurden 33 Patienten eingeschlossen (davon 21 Frauen, 12 Männer).

Schlussfolgerungen: Erste Schlussfolgerung zum praktischen Einsatz von Solriamfetol bei Narkolepsie werden präsentiert werden.

Schlüsselwörter: Narkolepsie, Tagesschläfrigkeit, Epworth Sleepiness Scale, Solriamfetol, Praktische Erfahrungen

\section{P 31}

\section{Narcolepsy treatments: comparison of pitolisant, modafinil and sodium oxybate via a network meta-analysis}

${ }^{*}$ C. Caussé', P. Lehert ${ }^{2,3}$

${ }^{1}$ Bioprojet, Medical, Paris, France; ${ }^{2}$ University of Louvain, Faculty of Economics, Mons, Belgium; '3niversity of Melbourne, Faculty of Medicine, Melbourne, Australia

Objectives: Narcolepsy is characterized by the excessive daytime sleepiness (EDS) measured by the Epworth Sleepiness Scale (ESS) and/or the Maintenance Wakefulness Test (MWT) and the cataplexy attacks rate (CAR). We performed a Multiple-Treatment comparison of three main treatments on EDS, CAR and Safety in randomized clinical trials (RCTs) through a Network meta-analysis (NMA).
Methods: A protocol was prepared according to meta-analyses guidelines. Study selection was all RCTs conducted in adult narcoleptic patients (ANP) assessing at least one of the 3 treatments and placebo. Univariate meta-analyses were performed separately for ESS, MWT and CAR, and confirmed by a multivariate comparison based on an agregating Z-score. Safety index was the Adverse Events incidence (AEI). Finally, a Risk/Efficacy index was calculated as a summary mean of Efficacy and Safety Zscore. A NMA was needed due to the multiple treatment comparison and multi-arm studies needing correction on correlation matrix. The random model was assumed due to the expected between studies heterogeneity.

Results: In ANP, Modafinil/Armodafinil (MDF), sodium oxybate (SXB) and Pitolisant $(\mathrm{P})$, demonstrated their efficacy to reduce EDS; $\mathrm{P}$ and SXB demonstrated their efficacy to reduce CAR. 14 studies were included in the NMA, 6 treatments groups were considered: MDF 200-400 mg/d, SXB $6 \mathrm{~g} / \mathrm{d}$ (SXB6) and $9 \mathrm{~g} / \mathrm{d}$ (SXB9) and P up to $20 \mathrm{mg} / \mathrm{d}$ (P20) and up to $40 \mathrm{mg} / \mathrm{d}$ (P40), and placebo. Placebo was considered as the reference value. SXB9, MDF and P40 were significantly different from placebo for ESS, MWT and CAR. In the ranking of treatments performed according to P-scores (Equivalent Sucra Values) and for all the efficacy endpoints confirmed by the multivariate meta-analysis, the highest treatment effect was found for P40, followed by SXB9 and MDF. The best safety profile compared with placebo was for Pitolisant (all doses), followed by Modafinil. SXB was characterized by a higher AEI. Finally, the best Risk/Benefit index was observed with P40.

Conclusions: This network meta-analysis provides evidence of the superiority of Pitolisant up to $40 \mathrm{mg} / \mathrm{d}$ over Modafinil or Sodium Oxybate on efficacy (EDS, CAR) and safety.

Disclosure: Bioprojet pharma sponsored this analysis. C. Caussé is an employee of bioprojet pharma. P. Lehert received honoraria from bioprojet. Keywords: Narcolepsy, Network meta-analysis, Pitolisant, Modafinil, Sodium Oxybate 


\section{Autorenverzeichnis}

\begin{tabular}{|c|c|c|c|c|c|}
\hline A & & De Jaegere, K. & 18, KV 11 & $\mathrm{H}$ & \\
\hline Aeschbach, D. & $9, \mathrm{KV} 1$ & de Vries, N. & P 28 & Hagmeyer, L. & KV 15 \\
\hline Ahmed, M. & KV 22 & Debener, $\mathrm{S}$. & P1 & Hamer, $\mathrm{O}$. & 16 \\
\hline Aigner, C. & P 25 & Debl, K. & 16 & Hartmann, M. & KV 7 \\
\hline Aironi, C. & P 21 & Deen, A. & P 24 & Hedner, J. & $6,7, \mathrm{KV} 22, \mathrm{KV} 23$ \\
\hline Akram, U. & P 22 & Dellweg, D. & KV 24 & Heidbreder, A. & 11 \\
\hline Anduleit, N. & KV 15 & Dietz-Terjung, S. & KV 10, KV 13, P 19, P 26 & Heiser, C. & KV 20 \\
\hline Arzt, M. & $1,16, \mathrm{KV} 26$ & Dillenhöfer, S. & KV 13 & Hennecke, E. & $\mathrm{KV} 1$ \\
\hline Asin, J. & P 28 & Doevelaar, A. & KV 8 & Herkenrath, $\mathrm{S}$. & $6,7, \mathrm{KV} 15$ \\
\hline Attali, V. & KV 21 & Dokic, D. & KV 21 & Hertel, D. & P6 \\
\hline & & Domanski, U. & KV 9 & Hetzenecker, A. & 16 \\
\hline & & Donjacour, C. & P 28 & Hofauer, B. & KV 20 \\
\hline B & & Dorffner, G. & KV 7 & Hohenhorst, W. & KV 12 \\
\hline Babel, N. & KV 8 & Dorn, $\mathrm{H}$. & P5 & Holub, F. & P 22 \\
\hline Bahr, K. & P 13 & & & Horvath, C.M. & $8, \mathrm{KV} 11$ \\
\hline Baladi, M. & $3, \mathrm{KV} 22, \mathrm{KV} 23$ & & & & \\
\hline Bartels, S. & 9,12 & E & & & \\
\hline Basoglu, Ö. & 6,7 & Ecker, A. & P 14 & I & \\
\hline Bauer, A. & KV 1 & Eckstein, L. & P 23 & In-Albon, T. & KV 18 \\
\hline Bauer, $\mathrm{F}$. & KV 8 & Eggert, T. & P5 & Ipsiroglu, $\mathrm{O}$. & KV 17 \\
\hline Baur, D. & KV 1 & Ell, J. & P9 & & \\
\hline Belev, G. & KV 21 & Elmenhorst, D. & KV 1 & & \\
\hline Bergmann, C. & KV 12 & Elmenhorst, E.-M. & KV 1 & J & \\
\hline Besa, V. & P 25 & Ernst, B. & P 13 & Janott, C. & KV 12 \\
\hline Birk, R. & P 16 & & & Jansen, V. & KV 9 \\
\hline Bleichner, M.G. & P1 & & & Janssen, $\mathrm{H}$. & P 28 \\
\hline Blome, C. & 5 & F & & Johann, A. F. & $P 22$ \\
\hline Bloutian, $\mathrm{H}$. & KV 12 & Faber, J. & P 10, P6 & & \\
\hline Bonsignore, M. & 6,7 & Farid-Moayer, M. & KV 24 & & \\
\hline Bosy-Westphal, A. & 5 & Feige, B. & $4, P 22, P 9$ & $\mathrm{~K}$ & \\
\hline Brandmayr, G. & KV 7 & Fellner, C. & 16 & Kallweit U & P 17, P 30 \\
\hline Brato, S. & P 19 & Ferrario, D. & KV 11 & Kamler M. & $\begin{array}{l}17, r 50 \\
\end{array}$ \\
\hline Braun, W. & 5 & Ficker, J.H. & 1, KV 24, KV 26 & Kamer, IVI. & KV 5 \\
\hline Braun, F. & 18, KV 11 & Fietze, I. & 1, KV 21, KV 26 & Kasngar, A. & $\begin{array}{l}\text { KVS } \\
\text { KV6 }\end{array}$ \\
\hline Braun, M. & P 26 & Fischer, C. & P7 & $\begin{array}{l}\text { Kater, IM.-J. } \\
\text { Kelher O. }\end{array}$ & $\begin{array}{l}K V 6 \\
P ?\end{array}$ \\
\hline Brill, A.-K. & 18, KV 11 & Fisser, C. & 16 & Kelber, O. & $P 2$ \\
\hline Bron, $M$. & KV 22 & Foldvary-Schaefer, N. & KV 22 & Kelmanson, I. & KV 14 \\
\hline Buchner, S. & 16 & Fredriksson, S. & 12 & Kim, J.-L. & 12 \\
\hline Büchner, N. & KV 8 & Friedlander, R. & KV 17 & Kirschbaum, C. & KV 4 \\
\hline Bujanover, S. & KV 23 & Frisch, D. & KV 19 & Kleibrink, B. & P 25 \\
\hline Buyse, B. & KV 21 & Fronczek-Poncelet, J. & KV 1 & Klofat, B. & P 11 \\
\hline & & & & Kluge, T. & KV 7 \\
\hline & & & & Knaack, L. & KV 24 \\
\hline C & & & & Koch-Hogrebe, M. & 15 \\
\hline $\mathrm{C}$ & & G & & Koerner-Rettberg, C. & KV 13 \\
\hline Carter, L. & KV 23, P 28 & Gaiduk, M. & KV 16, KV 3, P 21 & Kolb, C. & P2 \\
\hline Caussé, C. & KV 21, P 27, P 29, P 31 & Galetke, W. & KV 24, KV 9 & Koss, K. & P 5 \\
\hline Cauza, E. & P $14, P 15$ & Georgiev, 0 . & KV 21 & Krefting, D. & 11 \\
\hline Chandler, P. & $3, \mathrm{KV} 22$ & Gerlach, P. & P 11 & Krol, M. & P 14, P 15 \\
\hline Chen, D. & P 28 & Göder, R. & 5 & Kutz, P. & 15 \\
\hline Cicak, J. & P 15 & Götsch, M. & P5 & Kyle, S.D. & 4 \\
\hline Conti, M. & P 21 & Götz, K. & 16 & & \\
\hline Covenas, R. & KV 2 & Götze, J. & P 19 & & \\
\hline Cucu, R. & 18 & Gouveris, $\mathrm{H}$. & P 13 & L & \\
\hline Czellnik, A. & P 10 & Graml, A. & $1, \mathrm{KV} 26$ & Lammers, G.J. & KV 23, P 28 \\
\hline & & Grimm, J. & P 8 & Landolt, H.-P. & KV 1 \\
\hline & & Grote, L. & 6,7 & Lange, D. & KV 1 \\
\hline D & & Gruber, W. & KV 13 & Lange, C. & P 23 \\
\hline da Silva Souto, C. F. & P1 & Guenther, M. & P5 & Lechinger, J. & 5 \\
\hline Danker-Hopfe, H. & KV 5,P 5 & Gümüsdagli, F.E. & P 12 & $\begin{array}{l}\text { Lecninger, J. } \\
\text { Lecomte, l. }\end{array}$ & KV 21 \\
\hline Dauvilliers, Y. & KV 21, KV 23 & & & Lecomte, J.-M. & KV 21 \\
\hline
\end{tabular}




$\begin{array}{ll}\text { Lee, L. } & 3, \text { KV } 22 \\ \text { Lehert, P. } & \text { P 27, P 29, P } 31 \\ \text { Lemay, M. } & 18, \text { KV } 11 \\ \text { Lemkaddem, A. } & 18, \text { KV } 11 \\ \text { Lévy, P. } & \text { KV } 21 \\ \text { Li, H. } & \text { P } 16 \\ \text { Lieberich, L. } & \text { P } 30 \\ \text { Lin, C.-M. } & \text { KV } 24 \\ \text { Linker, C.-M. } & \text { P } 19 \\ \text { Llera-Perez, T. } & \text { P 23 } \\ \text { Lohaus, A. } & \text { KV 6 } \\ \text { Lollies, F. } & 10 \\ \text { Luigart, R. } & \text { KV 7 }\end{array}$

\section{M}

Maier, L. $\quad 16$

Malhotra, A. 3, KV 22, KV 23

Mann, C. P 18

Martin, A. KV 10

Martinez Madrid, N. KV 3, P 21

Matthiesen, I. $\quad \mathrm{P} 1$

Maurer, J.T. $\quad$ KV 20, P 3

Mayer, G. $\quad 3,11$, KV 22

Mazzini, S. P5

McNicholas, W. $\quad 6,7$

McWilliams, S. KV 17

Mellies, U. $\quad$ KV 13

Mevers, C.U. P30

Meyer, A. P 10

Müller, M. 5

Müller, C.E. P $\quad$ P 16

N

Naser Moghadasi, A. P 20

Nilius, G.

KV 9, KV 24, P 17

0

$\begin{array}{ll}\text { Ögren, M. } & 12 \\ \text { Olivier, M. } & \text { KV 13 } \\ \text { Orcioni, S. } & \text { P 21 } \\ \text { Ott, S. R. } & 18, \text { KV 11 } \\ \text { Overeem, S. } & \text { P 28 }\end{array}$

\section{P}

Partinen, M. KV 21

Pataka, A. $\quad 6,7$

Pätzold, W. P1

Paul, M. P1

Penz, M. KV 4

Penzel, T. KV 16, KV 3

Pépin, J.L. $\quad 6,7$, KV 21, KV 22, KV 23

Persson Waye, K. $\quad 12$

Pfeifer, M. $\quad 16$

Pietrowsky, R. P12

Poschenrieder, F. 16

Proença, M. KV 11

Q

Quehl, J. $\quad 9$

\begin{tabular}{ll|ll} 
R & & Stuck, B.A. & P 16 \\
Ramaekers, J. & P 28 & Sutharsan, S. & KV 13 \\
Randerath, W.J. & 6,7, KV 15, KV 24 & \\
Renevey, P. & 18, KV 11 & & \\
Rezaali, S. & P 20 & T & \\
Riemann, D. & 4, P 22, P 9 & Tamisier, R. & KV 21 \\
Rohn, B. & KV 8 & Tamm, S. & 4 \\
Roll, C. & 15 & Taube, C. & KV 10, KV 13, P 19, P 25, P 26 \\
Rosenberg, R. & 3 & Thees, R. & P 23 \\
Roth, C. & 18, KV 11 & Theurillat, P. & KV 11 \\
Roth, J. & P 18 & Thomas, T.A. & P 12 \\
Rothe, N. & KV 4 & Thyssen, J. & P 25 \\
Rupprecht, A. & P 23 & Toma, D. & KV 8 \\
Rutter, M. K. & 4 & Traxdorf, M. & P 7, P 8 \\
Ryan, S. & 6,7 & Treml, M. & 6,7, KV 15 \\
& Triller, A. & P 30 \\
& Trümper, D. & P 17 \\
S & Tscharaktschiew, N. & P 23 \\
Saalwirth, C. & 8 & Tschesche, A. & KV 9 \\
Saaresranta, T. & KV 21 & Tziridis, K. & P 7, P 8 \\
Sahraian, M. A. & P 20 & \\
Sarmiento, K. & KV 22 & & \\
Sauter, C. & KV 5, P 5 & & \\
Sche & &
\end{tabular}

Sauter, C. KV 5, P 5

\begin{tabular}{ll|l} 
Schiel, J.E. & 4 & Ulrich-Merzenich, G.
\end{tabular}

Schiza, S. $\quad 6,7$

Schlarb, A. A. $\quad 10$, KV 6, P 10, P 6

Schlüter, B. $\quad 15$

Schmelzer, K. $\quad$ KV 4

Schmieder, K. P5

Schnatschmidt, M. 10

Schneider, $\mathrm{H} . \quad \mathrm{KV} 24$

Schneider, B. KV 7

Schnepf, J. $\quad 1$, KV 26

Schöbel, C. $\quad 1$, KV 10, KV 26, P 19, P 25, P

26

Schröder, M. KV 9

Schubert, T. P 17

Schulze, $H . \quad$ P 7, P 8

Schwab, R. KV 22

Schwartz, J.-C. KV 21

Schwarze, J. P 10

Schweitzer, P. $\quad 3, \mathrm{KV} 23$

Seepold, R. KV 16, KV 3, P 21

Seibert, F.S. $\quad$ KV 8

Seidel, M. KV 8

Sexton, C.E. $\quad 4$

Shapiro, C. KV 22

Shcherbakova, A. P2

Sliwinski, $P . \quad 6,7$

Sommer, J.U. KV 20

Sommerwerck, U. P 25

Sophia, B. P 16

Specht, M. B. KV 24

Spiegelhalder, K. 4, P 22, P 9

Stadler, $S . \quad 16$

Stark, R. KV 5

Staubach, P. $\quad$ P 18

Steffen, A. KV 20

Stehling, F. KV 13

Steinmetz, L. P 22

Stier-Jarmer, M. KV 19

Stoohs, R. KV 24

Straßburg, S. P 19

Strohl, K.P. $\quad 3$, KV 23

Strollo, P.J. KV 22, KV 23

Stuburić, K. KV 3
V

Van Zaen, J. $\quad 18, \mathrm{KV} 11$

Verbraecken, J. $\quad 6,7, \mathrm{KV} 21$

Vermeeren, A. P 28

Verse, T. KV 20

Vetten, C. P 30

Vinckenbosch, F. P 28

Vogel, S. KV 4

Vogelmann, T. P 17

Vonk, P. P 28

W

Walther, A. KV 4

Wang, Y.-P. KV 24

Wang, S. KV 8

Wang, G. P 28

Weaver, T.E. KV 22

Weber, F. KV 5

Weeß, H.-G. KV 18, P 18

Wegener-Panzer, A. 15

Wegner, J. P 18

Weigt, L. P5

Weinhold, S.L. $\quad 5$

Weinreich, G. KV 10, P 19, P 25, P 26

Wekenborg, M. K. KV 4

Welsner, M. KV 10, KV 13, P 19

Wensley, D. KV 17

Werner, F.-M. KV 2

Werner, A. KV 6

Westhoff, T.H. KV 8

Witt, R. KV 18

Wittich, M. $\quad 5$

Wöhrle, H. 1, KV 26

Wolf, I. P1

Y

Young, P. $\quad 1, \mathrm{KV} 26$ 


\section{Autorenverzeichnis}

Z

Zeman, F. $\quad 16$

Zhou, K. P28 